No. 36.-JANUARY, 1906

DEPARTMENT OF THE INTERIOR

BUREAU OF GOVERNMENT LABORATORIES

\title{
A HAND-LIST OF THE BIRDS OF THE PHILIPPINE ISLANDS
}

\author{
BY \\ RICHARD C. MCGREGOR \\ AND \\ DEAN C. WORCESTER
}

MANILA

BUREAU OF PRINTING 


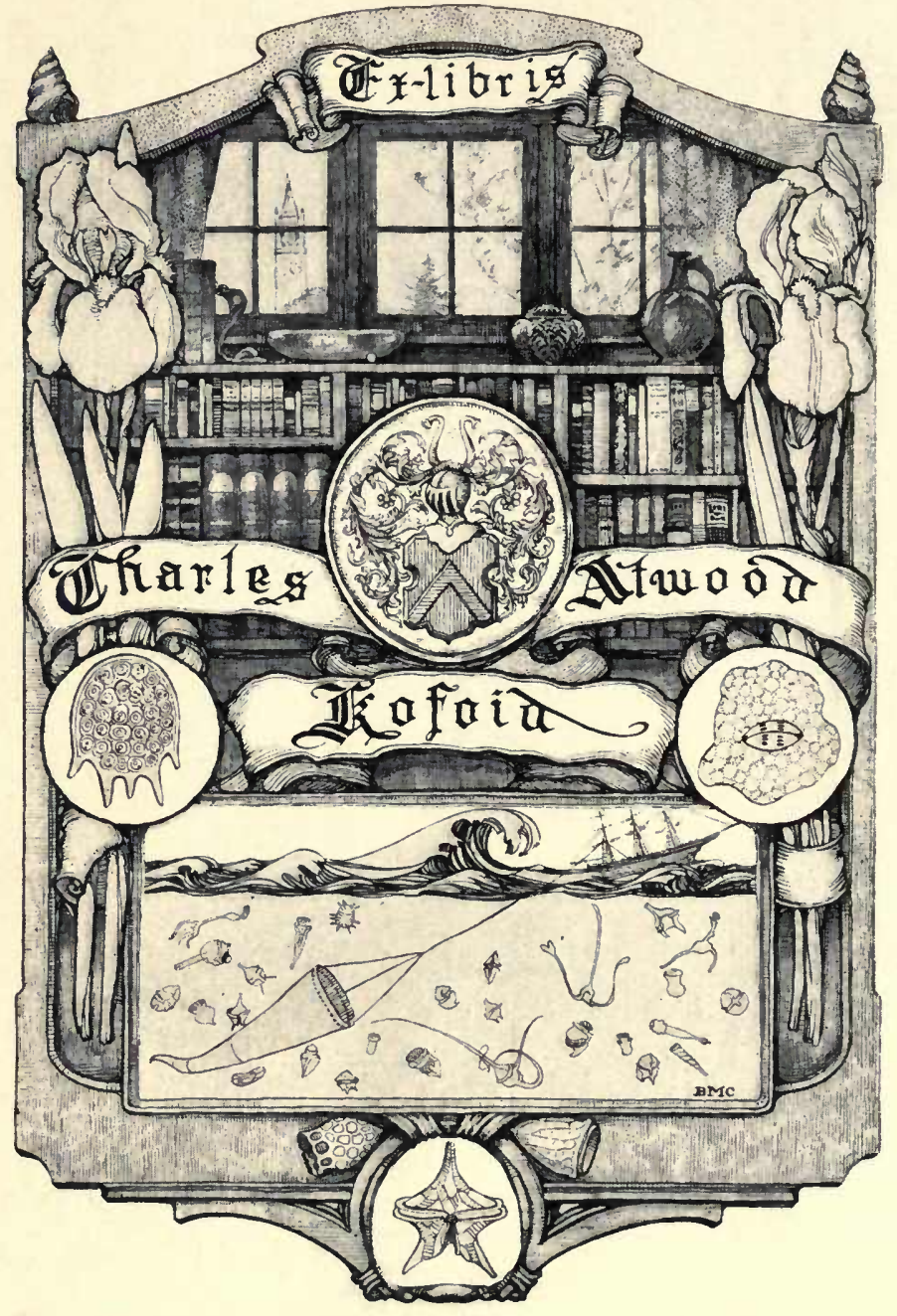




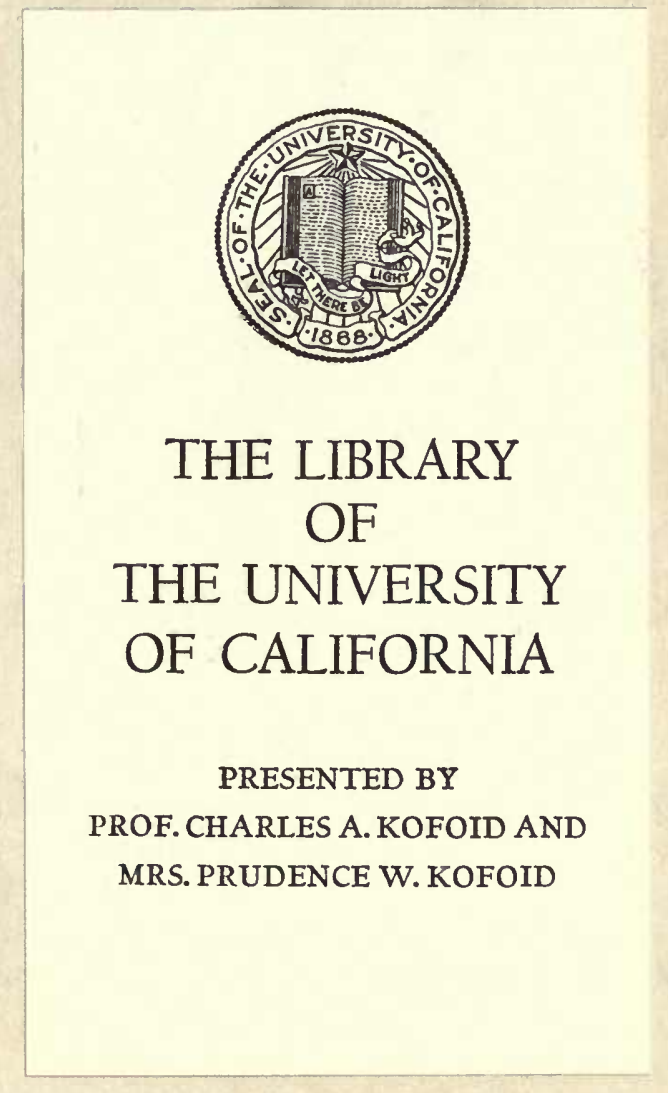




\section{E R R A T A.}

Page 4. In line 14 for loriculus read Loriculus.

Page 13. For STREPOPELIA read STREPTOPELIA.

Page 14. In line 4 for humulis read humilis.

Page 67. In lines 24 and 35 of footnote for Clark read Clarke.

Page 67. In last line of footnote for this read the former.

Page 101. For LIMONDROMUS read LIMONIDROMUS. 


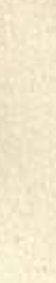




\title{
A HAND-LIST OF THE BIRDS OF THE PHILIPPINE ISLANDS
}

BY

\author{
RICIIARD C. MCGREGOR \\ AND
}

DEAN C. WORCESTER 


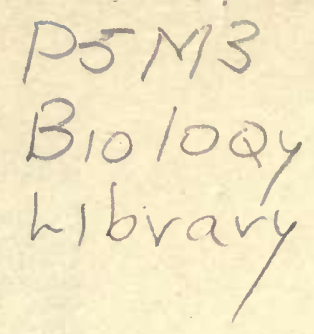

\section{INTRODUCTION}

'T'he appearance of the first volume of Dr. R. B. Sharpe's admirable "Hand-List of Birds" suggested to me the desirability of preparing a list, similar in form, of the birds of the Philippine Islands. The need of such a list was especially great in view of the fact that among the Americans seattered through every provinee of the Philippine Archipelago there were a number of members of the American Ornithologists's Union who were attempting to take up the study of Philippine birds, as well as numerous amateur ornithologists, whose attention was attracted by the unusual opportunities for ornithologieal work and who desired to identify the birds to be found in the vicinity of their several stations.

While the necessary literature for the identification of the known species of Philippine birds is on file in the general scientific library of the Bureau of Government Laboratories, its use by persons unfamiliar with Philippine orinithology is naturally attended with some practical difficulties. It therefore seemed to me highly desirable that there should be prepared a hand-list giving references to descriptions of all species known to inhabit the Philippines and stating accurately the known distribution of each species within the Archipelago. I began work upon such a list four years ago, but manifold official duties prevented rapid progress.

When the services of Mr. Richard C. McGregor were secured as collector of natural-history specimens this work, which had then been little more than begun, was turned over to him, as was an unpublished manuscript prepared by Dr. Frank S. Bourns and myself, from which it was loped that he would be able to derive information of value. It is only fair to Mr. McGregor to say that from that time until August 15, 1905, on which date he left for a well-earned rest in the United States, most of the work upon the Hand-List was performed by him. My own subsequent connection with it has been confined to consultation with him on doubtful points and to the final editing of the manuscript. As some of the footnotes were written by Mr. MeGregor prior to his departure and others which he has had no opportunity to see have been subsequently written by me, I lave deemed it desirable to give, in each instance, the authorship of the footnotes.

It has been our purpose to include all species which are known to 
inhabit the geographical area known as the Philippine Islands, as fixed by the treaty of Paris and the supplementary purchase of islands by the United States Government. The species of the Palawan Islands (Balabac, Palawan, Calamianes, and Cuyos groups) have accordingly been included.

The Distribution List of Philippine Birds prepared by Dr. Frank S. Bourns and myself (Proceedings of the United States National Museum. Vol. XX, pp. 549-566, 1898) enumerated 243 genera and 596 species for this same area. The work of Mr. John Whitehead, Dr. Edgar A. Mearns, Mr. Walter Goodfellow, Mr. John Waterstradt, and of Mr. McGregor and his Filipino assistants, together with such fragmentary work as I myself have been able to carry on in connection with official trips through the Islands, has raised this total to 284 genera, 691 identified species, and two species (Oceanodroma sp. and loriculus sp.) not identified. Some knowledge has been gained of the avifaunæ of the Islands of Ticao, Lubang, Cagayancillo, Agutaya, Calayan, Cresta de Gallo, Maestro de Campo, Semirara, East Balud, West Balud, and Sibay, the birds of which were heretofore entirely unknown.

The highlands of Mindanao have yielded a number of most interesting new genera and species, and additional knowledge has been gained relative to the birds of Luzon, Mindoro, Masbate, Negros, Samar, Cuyo, Culion, Cagayan-Sılu, Fuga, Romblon, and Sibuyan.

From the information now available we may deduce the following conclusions relative to the zoölogical relationships of these several islands:

Ticao belongs with the central group (Panay, Guimaras, Negros, and Masbate) rather than with Luzon.

Lubang must be considered a detached fragment of Luzon, as it has a number of characteristic Luzon species and entirely lacks those which are especially characteristic of Mindoro. One species (A ethopyga rubrinota McGregor) is, so far as we at present know, confined to this island.

On Cagayancillo is found Cinnyris aurora, but no other of the characteristic Palawan forms are known to exist there, while the occurrence of such species as Centropus viridis, Hypotaenidia torquata, Rallina euryzonoides, Limnobeanus fuscus, and Hierococcyx fugax, and the conspicuous absence of many of the species which are most common on the neighboring islands, lead to the conclusion that Cagayancillo is an oceanic island of recent formation and that its bird population is composed of the descendants of stragglers, most of which have probably come in from Negros or Mindanao, although $C$. aurora is evidently a wanderer from Cuyo or Palawan.

Agutaya belongs to the Palawan group.

Calayan has a strong Formosan element. Probably it will ultimately prove that the Batanes and Babuyanes Islands form a group by themselves.

Semirara belongs with Mindoro. 
The islands may therefore be divided into zoölogically distinct groups as follows:

(1) The Palawan group, consisting of Palawan and the small islands adjacent to it, Balabac, Cagayan Sulu, and the Cuyos and Calamianes Islands. The birds of this group show a very strong Bornean element. The line of demarcation between the Philippines, zoölogically speaking, and the Palawan Islands passes between Sibutu and the coast of Borneo and extends thence northward through the Sulu Sea east of the Cuyos group and through Mindoro Strait.

(2) The central Philippines, comprising the Islands of Negros, Panay, Guimaras, Masbate, and Ticao. 'They form a well-defined natural group, although in the case of Masbate and Ticao there are indications of immigration from Luzon.

(3) Mindoro and some of the islands immediately adjacent to it, including Semerara and doubtless also Ylin.

(4) Luzon, Catanduanes, Marinduque, and Lubang.

(5) Samar, Leyte, and Bohol. The Loriculus on Bohol proves to be Loriculus worcesteri and not Loriculus apicalis, while the presence of Orthotomus frontalis and Phabotrerom brevirostris among birds and of Galeopithecus philippinensis and Tarsius spectrum among mammals points unequivocally to the closest zoölogical relationship between Bohol and Leyte.

(6) Mindanao and the islands immediately adjacent to it form a group by themselves.

( $)$ Basilan must be separated from Mindanao on account of the fact that it has a number of representative forms of Mindanao species and lacks a number of species characteristic of Mindanao.

(8) Bongao, Tawi Tawi, Lapac, Siasi, and Sulu form a well-marked natural group, to which Sibutu must probably be added.

(9) Tablas, Romblon, and Sibuyan show no evidence of having been connected with any of the neighboring larger islands. They have a number of peculiar species of birds, and Tablas and Romblon should probably be classed together.

(10) Cebu can not be regarded as one of the central Philippine group, but must be classed by itself.

(11) The Batanes Islands have a strong Formosan element among their birds. It remains to be seen whether the Babuyanes Islands must be grouped with them or must be considered as detached fragments of northern Luzon.

(12) Siquijor, Cagayancillo, and Cresta de Gallo are islands of recent origin, and their bird faunæ have been derived from stragglers from neighboring islands.

While these several groups are by no means zoölogically equivalent, each has its highly characteristic species and forms a fairly natural division. 
In preparing the Hand-List no species has been recorded definitely from any. island except upon authority believed to be unimpeachable. Where doubt exists as to the oceurrence of a given species on any island the fact is indicated by an interrogation point placed after the name of such island. Several species for which no specific habitat within the Archipelago can be assigned have been included, upon the authority of the "Hand-List of Birds," in the belief that so eminent an authority as Dr. Sharpe would not have recorded them as occurring in the Philippines without sufficient evidence. On the other hand, a number of the Philippine species included in Sharpe's Hand-List have been excluded for reasons stated at the proper places in the text.

To and including the Family Certhiida the sequence of families is that of Sharpe's Hand-Iist, Volumes I to IV ; for the succeeding families we have followed the scheme proposed by Dr. R. V. Shufeldt in the American Naturalist, XXXVIII, pages 856-85\%. Thanks are due to Dr. Charles W. Richmond for suggested corrections in the nomenclature of various genera and species. Dr. Edgar A. Mearns has kindly furnished a MS. list of new localities for a number of species based upon his collections from Mindanao and neighboring islands.

'The work of the collectors regularly employed by the Insular Government, as well as that of residents of the Islands and of collectors representing scientific institutions of other countries, will doubtless make frequent additions and corrections necessary if this Hand-List is to be kept up to date. Supplemental pages containing addenda will be published from time to time until sufficient matter has been accumulated to justify the revision of the entire list.

It is hoped that students of Philippine ornithology will aid us, not only by calling attention to any errors which they may note in this list but by communicating authentic facts relative to the occurrence in the Philippines of species not included in it, and to the recording from any new islands in the Archipelago of species already known to inhabit the Philippines. Due credit will be given to all persons furnishing such information.

Attention is invited to the fact that the ornithological books and papers in the general scientific library of the Bureau of Government Laboratories ${ }^{1}$ and the bird collection of some 6,000 carefully identified specimens may be used, under reasonable restrictions, by any properly accredited person.

Deax C. Worcester.

Manima, P. I., September $9,1905$.

${ }^{1}$ The Bureau of Government Laboratories, after November 1, 1905, will be known as the Burean of Science. 


\section{Class AVES. \\ Subclass CARINATÆ.}

\section{Order GALLIFORMES.}

Suborler MEGAPODII.

Family MEGAPODIIDAE.

MEGAPODIUS $Q$. \& $G$.

(Grant, Cat. Bds., XXII, p. 446. 1893.)

cumingi Dillw. (p. 449.)

Celebean Islands

Islands of northwestern Borneo

$\begin{array}{ll}\text { Balabac } & \text { Mindoro } \\ \text { Basilan } & \text { Palawan } \\ \text { Cagayanclllo } & \text { Romblon } \\ \text { Calayan } & \text { Samar } \\ \text { Cebu } & \text { Sibutu } \\ \text { Cresta de Gallo } & \text { Slbuyan } \\ \text { Fuga } & \text { Sulu } \\ \text { Luzon } & \text { Tablas } \\ \text { Marinduque } & \text { Tawl Tawi } \\ \text { Mindanao } & \text { Tleao }\end{array}$

Suborder PHASIANI.

Family PHASIANIDAE.

EXCALFACTORIA $B p$.

(Grant, Cat. Bds., XXII, p. 249, 1893.)

lineata (Scop.). (p. 253.)

\begin{tabular}{|c|c|}
\hline $\begin{array}{l}\text { Borneo } \\
\text { Sumatra }\end{array}$ & $\begin{array}{l}\text { Java } \\
\text { Australia }\end{array}$ \\
\hline Calamianes & Negros \\
\hline Cebu & Palawan \\
\hline Lubang & Panay \\
\hline Luzon & Samar \\
\hline Masbate & Sibuyan \\
\hline Mindanao & Sulu \\
\hline Mindoro & Ticao \\
\hline
\end{tabular}


GALLUS Briss.

(Grant, Cat. Bds., XXII, p. 343, 1893.)

gallus (Linn.). (p. 344.)

\begin{tabular}{ll} 
Malay Peninsula \\
Indian Peninsula \\
$\begin{array}{l}\text { Indo-Malayan } \\
\text { Indo-Chinese countries } \\
\text { Indo- }\end{array}$ \\
\cline { 2 - 2 } Balabac & Mindanao \\
Basilan & Mindoro \\
Bongao & Negros \\
Calamianes & Palawan \\
Calayan & Panay \\
Cebu & Romblon \\
Fuga & Samar \\
Guimaras & Sibuyan \\
Lubang & Siquijor \\
Luzon & Sulu \\
Marinduque & Tablas \\
Masbate & Ticao
\end{tabular}

POLYPLECTRON Temm.

(Grant, Cat. Bds., XXII, p. 353, 1893.)

napoleonis Less. (p. 361.)

Palawan

[nehrkorna Blàsius.]

\section{Order HEMIPODII.}

\section{Family TURNICIDE.}

\section{TURNIX Bonn.}

(Grant, Cat. Bds., XXII, p. 526, 1893.)

\begin{tabular}{|c|c|c|}
\hline fasciata (Temm.). (p. 535.) & $\begin{array}{l}\text { Calamianes } \\
\text { Cebu } \\
\text { Luzon } \\
\text { Masbate } \\
\text { Mindoro }\end{array}$ & $\begin{array}{l}\text { Negros } \\
\text { Palawan } \\
\text { Panay } \\
\text { Sibuyan }\end{array}$ \\
\hline ellata (Scop.). (p. 548.) & Luzon & \\
\hline
\end{tabular}

whiteheadi Grant, Hand-Book Game Birds, Luzon II, p. 276 (1896).

worcesteri McGregor, Bull. Phil. Mus., No. 4, Luzon p. 8 (1904).

suluensis Mearns, Proc. Biol. Soc. Wash., Sulu XVIII, p. 83 (1905). 


\section{Order COLUMBIFORMES.}

Suborder COLUMBE.

Family TRERONIDE.

Subfamily TRERONIN E.

SPHENOCERCUS Gray.

(Salvaduri, Cat. Bds., XXI, p. 4, 1893.)

formosx (Swinh.). (p. 13.)

Mountains of Formosa

Calayan

TRERON Vieill.

(Salvadori, Cat. Bds., XXI, p. 33, 1893.)

nipalensis (Hodgs.). (p. 34.)

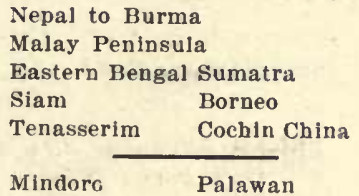

OSMOTRERON $B p$.

(Salvadori, Cat. Bds., XXI, p. 38, 1893.)

axillaris ( $B p$.$) (p. 48.)$

$\begin{array}{ll}\text { Basilan } & \text { Negros } \\ \text { Catanduanes } & \text { Panay } \\ \text { Cebu } & \begin{array}{l}\text { Romblon } \\ \text { Dinagat }\end{array} \\ \text { Gulmaras } & \begin{array}{l}\text { Semirara } \\ \text { Lubang }\end{array} \\ \text { Luzon } & \text { Siquijor } \\ \text { Masbate } & \text { Tawi Tawi } \\ \text { Mindanao } & \text { Ticao } \\ \text { Mindoro } & \text { Verde } \\ \text { Bongao } & \\ \text { Sibutu } & \text { Sulu } \\ & \\ \text { Celebes } & \\ \text { Malay Penlnsula } \\ \text { Indo-Malayan }\end{array}$

veretti Rothschild, Nov. Zool., I, p. 41 (1894).

vernans (Linn.). (p. 60.) 
PHAPITRERON $B p$.

(Salvadori, Cat. Bds., XXI, p. 66, 1893.)

amethystina $B p . \quad$ (p. 66.)

$\begin{array}{ll}\text { Dinagat } & \text { Mindanao } \\ \text { Leyte } & \text { Panaon } \\ \text { Luzon } & \text { Samar }\end{array}$

cinereiceps Bourns of Worcester, Occ. Papers Tawi Tawi Minnesota Acad., I, No. 1, p. 8 (1894).

brunneiceps Bourns of Worcester, $t$. c., p. 9. Basilan

frontalis Bourns of Worcester, t. c., p. 10 Cebu

maculipectus Bourns \& Worcester, t. c., p. 10. Negros

leucotis (Temm.). (p. 67.)

Catanduanes Luzon

Lubang Mindoro

occipitalis Salvad. (p. 68.) Basilan

nigrorum Sharpe. (p. 68.)

Cebu Panay

Guimaras Sibuyan

Masbate Tablas

Negros Ticao

brevirostris Tweedd. (p. 69.)

$\begin{array}{ll}\text { Bohol } & \text { Samar } \\ \text { Dinagat } & \text { Siquijor } \\ \text { Leyte } & \text { Sulu }\end{array}$

Subfamily PTILOPODIN正

\section{LEUCOTRERON $B p$.}

(Salvadori, Cat. Bds., XXI, p. \%1, 1893.)

occipitalis $B p$. (p. 72.)

\begin{tabular}{|c|c|c|}
\hline occipitalis $B p . \quad$ (p. 72.) & $\begin{array}{l}\text { Basilan } \\
\text { Cebu } \\
\text { Leyte } \\
\text { Luzon } \\
\text { Mindanao }\end{array}$ & $\begin{array}{l}\text { Mindoro } \\
\text { Negros } \\
\text { Samar } \\
\text { Sibuyan }\end{array}$ \\
\hline marchei (Oust.). (p. 75.) & Luzon & \\
\hline leclancheri $(B p$.$) . \quad (р. 79.)$ & $\begin{array}{l}\text { Cagayancillo } \\
\text { Calamianes } \\
\text { Calayan } \\
\text { Catanduanes } \\
\text { Cebu } \\
\text { Guimaras } \\
\text { Lubang } \\
\text { Luzon }\end{array}$ & $\begin{array}{l}\text { Mindoro } \\
\text { Negros } \\
\text { Palawan } \\
\text { Romblon } \\
\text { Samar } \\
\text { Semirara } \\
\text { Sibuyan } \\
\text { Tablas }\end{array}$ \\
\hline
\end{tabular}


LAMPROTRERON $B p$.

(Salvadori, Cat. Bds., XXI, p. 112, 1893.)

temmincki (Des Murs \&.Prév.). (p. 115.) Celebes

Sulu

SPILOTRERON Salvad.

(Salvadori, Cat. Bds., XXI, p. 141, 1893.)

bangueyensis (A. B. Mejyer). (p. 143.)

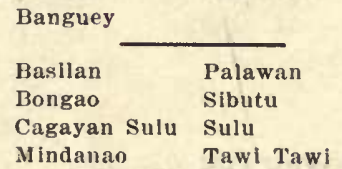

Subfamily CARPOPHAGIN

MUSCADIVORA Schlegel.

(Carpophaga Selby: Salvadori, Cat.

Bds., XX, p. 181, 1893.)

nuchalis (Cab.). (p. 190.)

$\begin{array}{ll}\text { Calayan } & \text { Mindanan } \\ \text { Fuga } & \text { Mindoro } \\ \text { Luzon } & \end{array}$

ænea (limn.). (p. 190.)

Indlan Peninsula

Ceylon

Indo-Chinese countries

Malay Peninsula

Sunda Islands

$\begin{array}{ll}\text { Balabac } & \text { Mindoro } \\ \text { Basilan } & \text { Negros } \\ \text { Bongao } & \text { Palawan } \\ \text { Calamianes } & \text { Panay } \\ \text { Catanduanes } & \text { Samar } \\ \text { Cebu } & \text { Semirara } \\ \text { Dinagat } & \text { Sibutu } \\ \text { Gulmaras } & \text { Sibuyan } \\ \text { Leyte } & \text { Siquljor } \\ \text { Lubang } & \text { Sulu } \\ \text { Luzon } & \text { Tablas } \\ \text { Marinduque } & \text { Tawi Tawi } \\ \text { Masbate } & \text { Tícao } \\ \text { Mindanao } & \text { Verde }\end{array}$

pickeringi (Cnss.) (p. 201.)

Mantanand Islands

Lawas River, northwest Borneo

Cagayanclllo Sibutu

Cagayan Sulu Sulu

langhornei Mearns, Proc. Biol. Soc. Wash., XVIII, p. 84 (1905). 
PTILOCOLPA $B p$.

(Salvadori, Cat. Bds., XXI, p. 205, 1893.)

carola Bp. (p. 206.)

Luzon

[griseipectus Bp. $=\delta ; C f$. Grant, Ibis,

Mindoro 1895 , p. 117 ; also Whitehead, Ibis, 1899 , p. 489.$]$

ndanao

Sibuyan

nigrorum Whitehead, Bull. B. O. C., VI, p. Negros $34(1897)$.

\section{ZONOPHAPS Salvad.}

(Salvadori, Cat. Bds., XXI, p. 20\%, 1893.)

poliocephala (Hartl.). (p. 209)

$\begin{array}{ll}\text { Basilan } & \text { Mindoro } \\ \text { Cebu } & \text { Negros } \\ \text { Dinagat } & \text { Panay } \\ \text { Leyte } & \text { Samar } \\ \text { Luzon } & \text { Sibuyan } \\ \text { Masbate } & \text { Tawi Tawi }\end{array}$

mindorensis (Whitehead), Ann. \& Mag. Nat. Mindoro

Hist., (6) p. 189 (1896). Fig., Grant, Ibis, 1896, pl. xi.

\section{MYRISTICIVORA Reichenb.}

(Salvadori, Cat. Bds., XXI, p. 22\%, 1893.)

bicolor (Scop.). (p. 227.)

\begin{tabular}{ll}
$\begin{array}{ll}\text { Andamans } \\
\text { Malay Peninsula }\end{array}$ & \multicolumn{1}{c}{ Nicobars } \\
Indo-Malayan & Islands \\
Moluceas & \\
Tenimber & Islands \\
Siam & Cochin China \\
\cline { 2 - 2 } Balabac & Negros \\
Bohol & Nipa \\
Bongao & Palawan \\
Cresta de Gallo & Sakuijok \\
Guimaras & Samar \\
Leyte & Sibay \\
Malanipa & Siquijor \\
Marinduque & Sulu \\
Masbate & Tawi Tawi \\
Mindanao & Ticao \\
Mindoro & West Bolod
\end{tabular}


Family COLUMBIDF.

Subfamily COLUMBINE.

COLUMBA Linn.

(Salvadori, Cat. Bds., XXI, p. 241,

1893.)

griseigularis (Wald. \& Layard). (p. 313.) Northern Borneo

\begin{tabular}{ll}
\cline { 2 - 2 } Basilan & Mindoro \\
Cagayan Sulu & Negros \\
Calayan & Romblon \\
Guimaras & Sibutu \\
Lubang & Sibuyan \\
Luzon & Sulu \\
Mindanao & West Bolod
\end{tabular}

Subfamily MACROPYGIIN

MACROPYGIA Swains.

(Salvadori, Cat. Bds., XXI, p. 335, 1893.)

tenuirostris $B p$. (p. 346.)

$\begin{array}{ll}\text { Balabac } & \text { Mihdoro } \\ \text { Basilan } & \text { Negros } \\ \text { Bongao } & \text { Palawan } \\ \text { Leyte } & \text { Romblon } \\ \text { Luzon } & \text { Sibutu } \\ \text { Marinduque } & \text { Sibuyan } \\ \text { Masbate } & \text { Sulu } \\ \text { Mindanao } & \text { Tawi Tawi }\end{array}$

phæa McGregor, Bull. Philippine Mus., No. 4, Calayan p. $9,1904$.

\section{Family PERISTERIDF.}

Subfamily TURTURIN E.

STREP0PELIA $B p$.

(Salvadori, Cat. Bds., XXI, p. 413, 1893.)

dussumieri (Temm.). (p. 423.)

Northern Borneo

Marianne Islands

\begin{tabular}{ll}
\cline { 2 - 2 } Agutaya & Mindoro \\
Basilan & Negros \\
Bohol & Palawan \\
Cagayancillo & Panay \\
Calayan & Romblon \\
Calamianes & Samar \\
Catanduanes & Semirara \\
Cebu & Siasi \\
Cuyo & Sibay \\
Guimaras & Sibutu \\
Leyte & Sibuyan \\
Lubang & Siquijor \\
Luzon & Sulu \\
Maestro de & Tablas \\
Campo & Tawi Tawi \\
Marinduque & Ticao \\
Masbate & Verde \\
Mindanao &
\end{tabular}


ONOPOPELIA Blanf.

(Blanf., Faun. Brit. Ind., Birds, IV, p. $4.7,1898$.

humulis (Temm.). (Salvad., Cat. Bds., XXI, p. 434.)

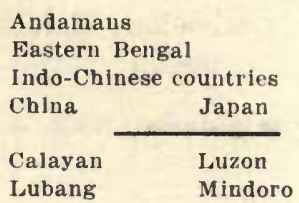

SPILOPELIA Sundev.

(Salvadori, Cat. Bds., XXI, p. 439 , 1893.)

tigrina ('Temm. de Knip.). (p. 440.)

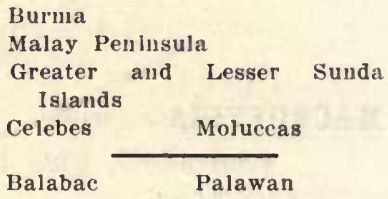

GEOPELIA Swains.

Subfamily GEOPELIIN无.

(Salvadori, Cat. Bds., XXI, p. 455, 1893.)

striata (Linn.). (p. 458.)

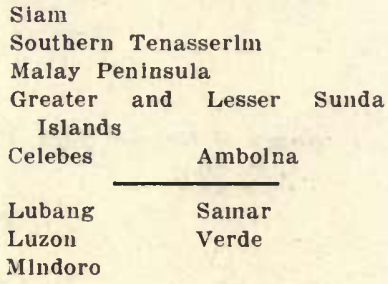

CHALCOPHAPS Gould.

Subfamily PHABIN E.

(Salvadori, Cat. Bds., XXI, p. 510, 1893.)

indica (Linu.). (p. 514.)

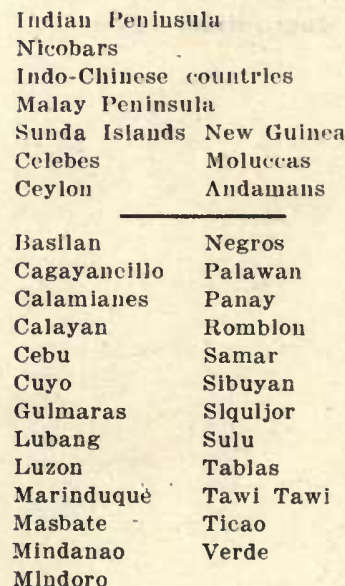


PHLOGGNAS Reichenb.

(Salvadori, Cat. Bds., XXI, p. 583, 1893.)

Iuzonica (Scop.). (p. 585.)

Luzon

crinigera (Jaeq. \& Pucher.). (p. 587.)

$\begin{array}{ll}\text { Basilan } & \text { Samar } \\ \text { Leyte } & \text { Sulu? }\end{array}$

keayi Clarke, Ibis, 1900 , p. 359.

Negros

menagei Bourms \& Worcester, Occ. Papers

Tawi Tawi

Minnesota Acad., I, No. 1, p. 10 (1894).

platenæ Hartert. (p. 58s.)

Mindoro

Subfumily CALENADIN FE.

CALCENAS Gray.

(Salvadori, Cat. Bds., XXI, p. 614, 1893.)

nicobarica (Linn.). (p. 615.)

Nicobar Islands
Mergui Archipeiago
Greater Sunda Islands
Bismarck Archipelago
Moluceas to New Guinea
$\begin{array}{lc}\text { Luzou } & \text { Sibutu } \\ \text { Mindoro } & \text { Sulu } \\ \text { Negros } & \text { Tawl Tawi } \\ \text { Palawan } & \end{array}$

\section{Orler RALAIFORMES.}

\section{Family RALLIDAE.}

Subfamily RALLIN E.

HYPOT ENIDIA lieichenb.

(Sharpe, Cat. Bds., XXIII p. 32, 1894.)

striata (liml.). (p. 33.)

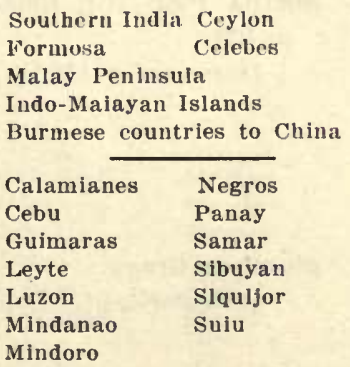

${ }^{1}$ See Pucher. ¿f Jacq., Voy. l'ǒle Sud. I11, Ois., p. 18 (1853). P. crinigera was not met witl in Sulu by Platen or by Dr. Bourns and myself.-Worcester. 
philippinensis (Linn.). (p. 39.)

torquata (Linn.). (p. 43.)
Pacific Islands

Australia

New Zealand

Entire Malayan Archipelago

Luzon

$\begin{array}{ll}\text { Bongao } & \text { Mindanao } \\ \text { Cagayancillo } & \text { Mindoro } \\ \text { Catanduanes } & \text { Negros } \\ \text { Cebu } & \text { Panay } \\ \text { Dinagat } & \text { Romblon } \\ \text { Guimaras } & \text { Samar } \\ \text { Leyte } & \text { Sibuyan } \\ \text { Luzon } & \text { Siquijor } \\ \text { Marinduque } & \text { Ticao } \\ \text { Masbate } & \text { Verde }\end{array}$

RALLINA Reichenb.

(Sharpe, Cat. Bds., XXIII, p. 74, 1894.)

fasciata (Raffl.). (p. 75.)

Burmese provinces

Malay Peninsula

Indo-Malayan Islands

Halmahéra Pelew Islands

Balabac Palawan

euryzonoides (Lafr.). (p. 78.)

$\begin{array}{ll}\text { Cagayancillo } & \text { Mindoro } \\ \text { Cebu } & \text { Negros } \\ \text { Leyte } & \text { Panay } \\ \text { Luzon } & \text { Sulu } \\ \text { Mindanao } & \end{array}$

PORZANA $V$.

(Sharpe, Cat. Bds., XXIII, p. 92, 1894.)

pusilla Pall. (Cf. Reichenb., J. f. O., 1898, p. 139.)

[intermedia (Herm.). (p. 103.)]

Central Europe

East to Lake Baikal

Africa and Madagascar (winter)

Luzon

plumbea Gray.

New Hebrides Australia

[tabuensis, pt. (pp. 111, 335.)]

New Caledonia

New Zealand

Chatham Islands

Samoa Isfands Fiji Islands

Luzon 
POLIOLIMNAS sharpe.

(Sharpe, Cat. Bds., XXIII, p. 130, 1894.)

cinereus $(V$.$) . (pp. 130, 337.)$

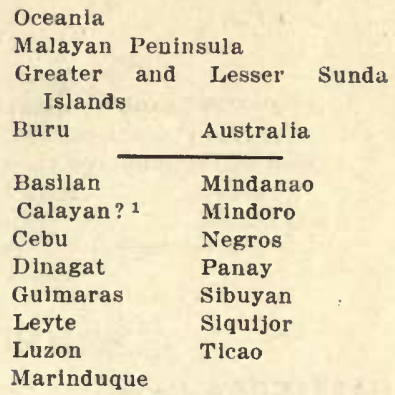

LIMNOBEENS Sundev.

(Sharpe, Cat. Bds., XXIII, p. 145, 1894.)

fuscus (Linn.). (p. 146.)

Indlan Peninsula
Ceylon
Burmese countries to China
and Japan
Malay Peninsula
Cbristmas Island
Java Borneo
$\begin{array}{ll}\text { Cagayancillo } & \text { Mindanao } \\ \text { Leyte } & \text { Mindoro } \\ \text { Luzon } & \text { Negros }\end{array}$

paykulli (l,junyh.). (1. 14!.)

Chiua
Malay Peninsula
Eastern Siberia
Java Borneo
Basilan

AMAUR0NIS Reichenb.

(Sharpe, Cat. Bds., XXIII, p. 152, 1894.)

olivacea (Meyen). (p. 153.)

$\begin{array}{ll}\text { Calayan } & \text { MIndoro } \\ \text { Cebu } & \text { Negros } \\ \text { Leyte } & \text { Panay } \\ \text { Luzon } & \text { Samar } \\ \text { Masbate } & \text { Slquijor } \\ \text { Mindanao } & \text { Ticao }\end{array}$

${ }^{1}$ A rail, probably $P$. cinereus, was seen but not obtained by Mr. McGregor in Calayan.-Worcester.

$35916-2$ 
phœnicura (Forster). (p. 156.)

Celebes
Indian Peninsula
Indo-Burmese provinces
$\begin{aligned} & \text { Malay Peninsula } \\ & \text { Greater and } \\ & \text { Islands } \\ & \text { Ceylon }\end{aligned}$
$\begin{array}{ll}\text { Basilan } & \text { China } \\ \text { Bongao } & \text { Mindoro } \\ \text { Cagayan Sulu } & \text { Palawan } \\ \text { Calamianes } & \text { Panay } \\ \text { Marinduque } & \text { Siquijor } \\ \text { Mindanao } & \text { Tawi Tawi }\end{array}$

GALIINULA Briss.

(Sharpe, Cat. Bds., XXIII, p. 16\%, 1894.)

chloropus (Linn.). (p. 169.)

\begin{tabular}{ll}
$\begin{array}{l}\text { Europe } \\
\text { Asia } \\
\text { Madagascar }\end{array}$ & $\begin{array}{l}\text { Africa } \\
\text { Mauritius }\end{array}$ \\
\cline { 2 - 2 } Calayan & Mindanao \\
Cebu & Mindoro \\
Guimaras & Negros \\
Leyte & Panay \\
Luzon & Samar
\end{tabular}

GALLICREX Blyth.

(Sharpe, Cat. Bds., XXIII, p. 183, 1894.)

cinerea (Lath.). (p. 183.)

\begin{tabular}{l} 
Ceylon \\
Burmese provinces to China \\
and Japan \\
Malay Peninsula \\
Indian PenInsula \\
Greater Sunda Islands \\
\cline { 2 - 2 } $\begin{array}{ll}\text { Catanduanes } & \text { Mindoro } \\
\text { Cebu } & \text { Negros - } \\
\text { Luzon } & \text { Panay } \\
\text { Marinduque } & \text { Sibuyan } \\
\text { Masbate } & \text { Sulu } \\
\text { Mindanao } & \text { Ticao }\end{array}$
\end{tabular}

PORPHYRIO Briss.

(Sharpe, Cat. Bds., XXII1, p. 192, 1894.)

pulverulentus T'emm. (p. 207.) 
FULICA Linn.

(Sharpe, Cat. Bds., XXIII, p. 209, 1894.)

atra Linn. (p. 210.)

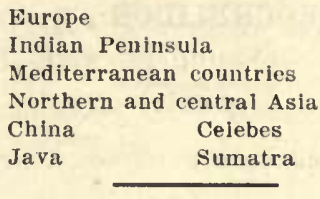

Luzon

\section{Order PODICIPEDIDIFORMES.}

Family PODICIPEDIDAE.

PODICIPES Lath.

(Grant, Cat. Bds., XXVI, p. 502, 1898.)

philippinensis (Bonn.). (p. 511.)

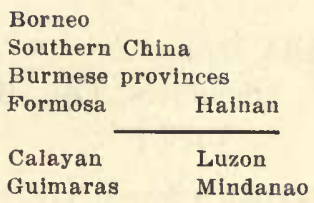

Order PROCLLLARIIFORMES.

Family PROCELLARIIDF.

Subfamily PROCELLARIINE.

OCEANODROMA Reichenb.

(Salvin, Cat. Bds., XXV, p. 347 , 1896.)

sp. McGregor, Bull. Philippine Mus., No. 4, Luzon p. 12 (1904).

\section{Family PUFFINIDAE. \\ Suhfamily PUFFININ}

PUFFINUS Briss.

(Salvin, Cat. Bds., XXV, p. 368, 1896.)

leucomelas Temm. (p. 370.)

Japan and Korea south to Austraila 


\title{
Order LARIFORMES.
}

Family LARIDAE.

Subfamily STERNINÆ:

\section{HYDROCHELIDON Boie.}

(Saunders, Cat. Bds., XXV, p. 5, 1891.)

leucoptera (Meisn. \& Schinz). (p. 6.)

hybrida (Pall.). (p. 10.)

\author{
Africa (winter) \\ Australia New Zealand \\ Central and southern Europe to \\ central Asia and China \\ Mindanao \\ Malay Archipelago \\ Australia Africa \\ Southwestern central, and \\ southern Europe to China

$\begin{array}{ll}\text { Luzon } & \text { Palawan } \\ \text { Mindanao } & \text { Negros }\end{array}$

STERNA Linn.

(Saunders, Cat. Bds., XXV, p. 40, 1896.)

fluviatilis Naum. (p. 54.)

Countries on both sides of Atlantic Ocean

Indian and African coasts (winter)

Brazilian coasts (winter)

Calayan

boreotis (Bangs), Bull. Mus. Comp. Zool.,

Red Sea

XXXVI, No. 8, 256 (1901).

African and Indian Oceans to China, Japan, Australia, and the Pacific Islands.

\begin{tabular}{|c|c|}
\hline Agutaya & Negros \\
\hline Balabac & Palawan \\
\hline Bohol & Panay \\
\hline Calamianes & Pata off Sulu \\
\hline Cebu & Romblon \\
\hline Cuyo & Samar \\
\hline Guimaras & Sibuyan \\
\hline Leyte & Siquijor \\
\hline Luzon & Sulu \\
\hline Masbate & Tablas \\
\hline Mindanao & Tawi Tawi \\
\hline
\end{tabular}

anæstheta Scop. (p. 101.)

African and Indian seas to China, Japan, Moluccas, northern Australia, and Pacific Islands.

Gulf of Mexico Western Indies Panay 
fuliginosa $\mathrm{Gm}$. (p. 106.)

sinensis $G m . \quad$ (p. 113.)

melanauchen Temm. (p. 126.)
Tropical and juxtatropical seas of the world

Siquijor

Chinese and Indian seas to
Australia

Mindanao Palawan

Mindoro

Tenasserim Andamans
Nicobars
Malay Peninsula
Northern Australia
Pacific Islands to Liu Kiu
Islands
Northern Mascarene Islands
Palawan Cresta de Gallo

ANOUS Steph.

(Saunders, Cat. Bds., XXV, p. 136 , 1896.)

stolidus (Linn.). (p. 136.)

Tropical and juxtatropical seas of the world

Cagayan Sulu Palawan

Subfamily LARIN无.

\section{LARUS Linn.}

(Saunders, Cat. Bds., XXV, p. 169 , 1896.)

ridibundus Linn. (p. 207.)

$\begin{aligned} & \text { Europe and northern Asia } \\ & \text { Africa Indian Ocean } \\ & \text { China to Malay Archipelago } \\ & \text { (winter) }\end{aligned}$
$\begin{array}{ll}\text { Luzon Samar } \\ \text { Mindanao }\end{array}$

vegæ Palmén. (p. 270.)

Chinese coasts (winter)

Japan and Formosa (winter)

Bonin Islands (winter)

Arctic Siberia

Bering Sea

Luzon 


\title{
Order CHARADRIIFORMES.
}

\author{
Suborder CHARADRII. \\ Family CHARADRIIDE.
}

Suḅfamily ARENARIIN EE.

ARENARIA Briss.

(Sharpe, Cat. Bds., XXIV, p. 91, 1896.)

interpres (Linn.). (p. 92.)

Cosmopolitan, breeding in high northern latitudes

$\begin{array}{ll}\text { Bohol } & \text { Masbate } \\ \text { Cagayancillo } & \text { Mindanao } \\ \text { Cagayan Sulu } & \text { Negros } \\ \text { Lubang } & \text { Palawan } \\ \text { Luzon } & \text { Siquijor }\end{array}$

Subfamily CHARADRIIN $F$.

SQUATAROLA Leach.

(Sharpe, Cat. Bds., XXIV, p. 182, 1896.)

helvetica (Linn.). (p. 182.)

Subaretic regions south to Australia, Cape, and South America (winter)

$\begin{array}{ll}\text { Cuyo } & \text { Negros } \\ \text { Luzon } & \text { Palawan } \\ \text { Mindanao } & \text { Siquijor }\end{array}$

CHARADRIUS Linn.

(Sharpe, Cat. Bds., XXIV, p. 191, 1896.)

fulvus $(G m$.$) . (p. 195.)$

[dominicus (P. L. S. Mïll.).]

Subarctic regions south to Australia

New Zealand Africa

South America (winter)

Balabac Masbate

Bohol Mindanao

Calamianes Mindoro

Calayan Negros

Cebu Palawan

Cuyo Sibay

Fuga Sibuyan

Leyte Siquijor

Lubang Sulu

Luzon Ticao 
OCHTHODROMUS Reichenb.

(Sharpe, Cat. Bds., XXIV, p. 209, 1896.)

geoffroyi (Wagl.). (p. 217.)

mongolus (Pall.). (p. 223.)

veredus (Gould). (p. 232.)

Eastern Asla (Japan, Formosa)

Africa, Indlan Peninsula, and A ustralla (winter)

$\begin{array}{ll}\text { Bohol } & \text { Mindanao } \\ \text { Cagayanclllo } & \text { Mindoro } \\ \text { Cuyo } & \text { Palawan } \\ \text { Leyte } & \text { Panay } \\ \text { Lubang } & \text { Pata off Sulu } \\ \text { Luzon } & \text { Siquijor } \\ \text { Negros } & \end{array}$

Northeastern Asia

Coasts of Berlng Sea, Alaska

Chlna to Australia (winter)

$\begin{array}{ll}\text { Bohol } & \text { Luzon } \\ \text { Cagayanclllo } & \text { Mindanao } \\ \text { Calayan } & \text { Negros } \\ \text { Cuyo } & \text { Palawan } \\ \text { Leyte } & \text { Ticao } \\ \text { Luhang } & \end{array}$

Mougolia

China to Moluccas and Australia (winter)

Palawan

ÆEGIALITIS Boie.

(Sharpe, Cat. Bds., XXIV, p. 254, 1896.)

dubia (Scop.). (p. 263.)

Africa (winter)

Alaska to Callfornla (casual)

Europe and northern Asia to Japan

Indla and the Malay Archlpelago (winter)

$\begin{array}{ll}\text { Bohol } & \text { Luzon } \\ \text { Cagayanclllo } & \text { Mlndanao } \\ \text { Calayan } & \text { Mindoro } \\ \text { Catanduanes } & \text { Negros } \\ \text { Cebu } & \text { Palawan } \\ \text { Gulmaras } & \text { Panay } \\ \text { Leyte } & \text { Sibuyan } \\ \text { Lubang } & \text { Tablas }\end{array}$

peroni (Bp.): (p. 273.)

Greater Sunda Islands to Celebes

\begin{tabular}{ll}
\cline { 2 - 2 } Bohol & Negros \\
Calayan & Palawan \\
Fuga & Romblon \\
Leyte & Sibutu \\
Lubang & Sibuyan \\
Luzon & Slquijor \\
Mindanao & Ticao
\end{tabular}


alexandrina (Linn.). (p. 275.)

Africa
Indian Peninsula and Australia
(winter)
Europe and central Asia to
China and Japan
$\begin{array}{ll}\text { Calayan } \\ \text { Cuyo } \\ \text { Mindanao } \\ \text { Palawan }\end{array}$

Subfamily HIMANTOPODINÆ.

HIMANTOPUS Briss.

(Sharpe, Cat. Bds., XXIV, p. 309, 1896.)

leucocephalus Gould. (p. 317.)

Molucca Islands

Greater. Sunda Islands

Australia New Guinea

Mindanao

Subfamily TOTANIN Æ.

NUMENIUS Briss.

(Sharpe, Cat. Bds., XXIV, p. 340, 1896.)

arquata (Linn.). (p. 34l.)

India Africa

Europe east to Lake Baikal

Southern China and Malay Peninsula (winter)

$\begin{array}{ll}\text { Luzon } & \text { Samar } \\ \text { Masbate } & \text { Ticao }\end{array}$

Palawan

cyanopus $V$. (p. 350.)

Japan

Eastern Siberia

Australia (winter)

Mindoro $\longdiv { \text { Negros } }$

variegatus (Scop.). (p. 361.).

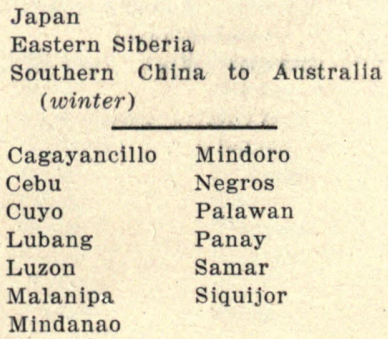


MESOSCOLOPAX Sharpe.

(Sharpe, Cat. Bds., XXIV, p. 371, 1896.)

minutus Gould. (p. 371.)

China

Eastern Siberla

Corea and Mongolia

Japan to Australla (winter)

Marlnduque Mindanao

LIMOSA Briss.

(Sharpe, Cat. Bds., XXIV, p. 372, 1896.)

novæ-zealandiæ Gray. (p. 379.)

Alaska

Eastern Siberla south to Australla

New Zealand, Oceania (winter)

Bohol

Cuyo

Negros

limosa (Linn.). (p. 391.)

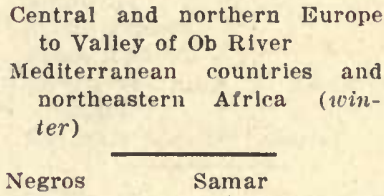

TOTANUS Bechst.

(Sharpe, Cat. Bds., XXIV, p. 409, 1896.)

eurhinus (Oberh.), Proc. U. S. N. M., XXII, p. 207 (1900).

Indla Africa
Eurape and central Asia to
eastern Slberia
Burmese countries to Malay
Archipelago (winter)
$\begin{array}{ll}\text { Bohol } & \text { Negros } \\ \text { Cebu } & \text { Palawan } \\ \text { Cuyo } & \text { Siquijor } \\ \text { Mindanao } & \end{array}$

HELODROMAS Kaup.

(Sharpe, Cat. Bds., XXIV, p. $43 \%$, 1896.)

ochropus (Linn.). (p. 437.)

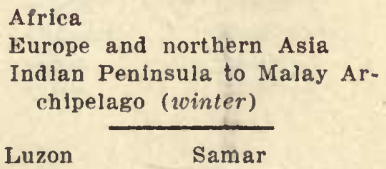


HETERACTITIS Stejn.

(Sharpe, Cat. Bds., XXIV, p. 449 , 1896.)

brevipes (V.). (p. 449.)

Eastern Siberia
China to Malay Archipelago
and Australia (winter)

ACTITIS Illiger.

(Tringoides Bp.; Sharpe, Cat. Bds., XXIV, p. 456, 1896.)

hypoleucus (Linn.). (p. 456.)

\begin{tabular}{ll}
$\begin{array}{l}\text { Africa } \\
\text { Europe and northern Asi } \\
\text { Indian Peninsula to Aust } \\
\text { (vinter) }\end{array}$ \\
\cline { 2 - 2 } Agutaya & Lubang \\
Balabac & Luzon \\
Basilan & Masbate \\
Bohol & Mindanao \\
Cagayancillo & Mindoro \\
Cagayan Sulu & Negros \\
Calamianes & Palawan \\
Calayan & Panay \\
Camiguin & Samar \\
Cebu & Sibay \\
Cuyo & Ticao \\
Guimaras & Verde
\end{tabular}

TEREKIA $B p$.

(Sharpe, Cat. Bds., XXIV, p. 474, 1896.)

cinerea (Güldenst.). (p. 474.)

Northern Siberia

Northeastern Europe

Africa and Indian Peninsula to Australia (winter)

$\begin{array}{ll}\text { Bohol } & \text { Negros } \\ \text { Masbate } & \text { Palawan }\end{array}$

GLOTTIS $K o c h$.

(Sharpe, Cat. Bds., XXIV, p. 480 , 1896.)

nebularius (Gunn.). (p. 481.)

Africa

Northern Europe and northern Asia

Indian Peninsula to Australia (winter)

$\begin{array}{ll}\text { Luzon } & \text { Negros } \\ \text { Mindanao } & \text { Samar } \\ \text { Mindoro } & \end{array}$


RHYACOPHILUS Kaup.

(Sharpe, Cat. Bds., XXIV, p. 490, 1896.)

glareola $(G m$.$) . (p. 491.)$

$\begin{array}{ll}\begin{array}{l}\text { Africa } \\ \text { Europe and northern Asia } \\ \text { Indian Peninsula to Australia } \\ \text { (winter) }\end{array} \\ \begin{array}{ll}\text { Bohol } \\ \text { Cagayan Sulu }\end{array} & \text { Mindanao } \\ \text { Calamianes } & \text { Negros } \\ \text { Calayan } & \text { Palawan } \\ \text { Cebu } & \text { Panay } \\ \text { Guimaras } & \text { Siquijor } \\ \text { Lubang } & \text { Ticao } \\ \text { Luzon } & \end{array}$

Subfamily sCOLOPACIN无.

CALIDRIS Illiger.

(Sharpe, Cat. Bds., XXIV, p. 526, 1896.)

abba (Pall.). (p. 526.)

[Cf. Richmond, Smithson, Miscel. Colls., 47 , p. 347 (1905).]

Africa

Arctic regions

South America

Indian Peninsula to Australia (winter)

Marshall Islands (winter)

Luzon

\title{
LIMONITES Kaup.
}

(Sharpe, Cat. Bds., XXIV, p. 53\%, 1896.)

minuta (Leister). (p. 538.)

\author{
Africa \\ Ceylon (winter) \\ Indian Peninsula \\ Northern Europe \\ Northern Asia to Lake Baikal \\ Mindanao
}

ruficollis (Pall.). (p. 545.)

\begin{tabular}{l}
$\begin{array}{l}\text { Eastern Siberia } \\
\text { Japan } \\
\text { Burmese countries to Aust } \\
\text { (winter) }\end{array}$ \\
\cline { 2 - 2 } $\begin{array}{l}\text { Bohol } \\
\text { Calayan }\end{array}$ \\
$\begin{array}{ll}\text { Cebu } & \text { Muzon } \\
\text { Cuyo } & \text { Negros } \\
\text { Palawan }\end{array}$
\end{tabular}


damacensis (Horsf.). (p. 553.)

temmincki (Leisl.). (p. 555.)

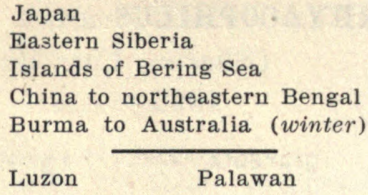

Negros

\title{
HETEROPYGIA Coues.
}

(Sharpe, Cat. Bds., XXIV, p. 561, 1896.)

acuminata (Horsf.). (p. 566.)

\author{
Eastern Siberia \\ Alaska \\ China south to Australia and \\ New Zealand (winter) \\ Mindanao
}

\section{ANCYLOCHILUS Kaup.}

(Sharpe, Cat. Bds., XXIV, p. 585, 1896.)

subarquatus (Güldenst.). (p. 586.)

\author{
Northern Siberia \\ Africa \\ Indian Peninsula to Australia \\ (winter) \\ Luzon Negros
}

TRINGA Linn.

(Sharpe, Cat. Bds., XXIV, p. 593, 1896.)

crassirostris T. \& S. (p. 600.)

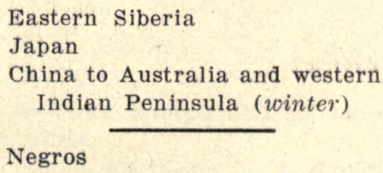


LIMICOLA Koch.

(Sharpe, Cat. Bis., XXIV, p. (61\%, 1896.)

platyrhyncha ('T'cmm.). (p. 612.)

Northern Europe and Siberia Mediterranean and Red Seas

Indlan Penlnsula

China to Moluccas (winter)

Bohol

Cuyo

Negros

Palawan

GALLINAG0 J,each.

(Sharpe, Cat. Bds., XXIV, p. 616, 1896.)

stenura (Kuhl). (p. 619.)

Eastern Siberla to the Yenesei China to Malay Peninsula (vinter)

Calayan Mlndanao

megala Swinh. (p. 624.)

Eastern Siberia

Japan

China south to Molucca Islands (winter)

\begin{tabular}{|c|c|}
\hline Basilan & MIndoro \\
\hline Calamianes & Negros \\
\hline Cebu & Palawan \\
\hline Leyte & Panay \\
\hline Lubang & Samar. \\
\hline Luzon & Sibuyan \\
\hline Masbate & Siquijor \\
\hline Mlndanao & Tawi Ta \\
\hline
\end{tabular}

gallinago (limn.). (p. 633.)

Europe to northern Asla

Senegambla and northeastern Africa (winter)

Indian Peninsula to the Malay Penlusula and the Moluceas (winter)

$\begin{array}{ll}\text { Bobol } & \text { Luzon } \\ \text { Leyte } & \text { Mindoro }\end{array}$

ROSTRATULA $V$.

(Sharpe, Cat. Bds., XXIV, p. 683, 1896.)

capensis (Linn.). (p. 683.)

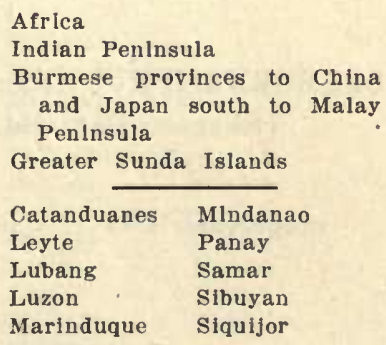


Suborder PARRÆ.

Family PARRIDAE.

HYDROPHASIS Sharpe.

(Sharpe, Cat. Bds., XXIV, p. 69, 1896.)

chirurgus (Scop.). (p. 69.)

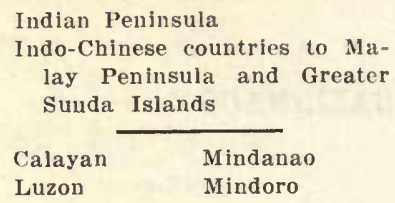

HYDRALECTOR Wagler.

(Sharpe, Cat. Bds., XXIV, p. 89 , 1896.)

gallinaceus (Temm.). (1. c.)

Australla

Celebes

Southern Borneo

Mindanao

Suborder CURSORII.

Family GLAREOLIDAE.

GLAREOLA Briss.

(Sharpe, Cat. Bds., XXIV, p. 53, 1896.)

orientalis Leach. (p. 58.)

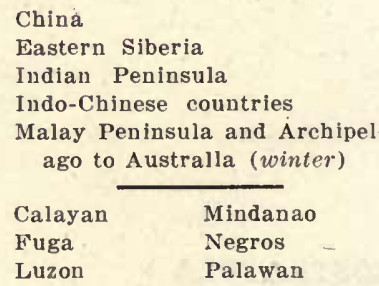

Suborder CEDICNEMI.

Family CEDICNEMIDAE.

ORTHORHAMPHUS Salvad.

(Sharpe, Cat. Bds., XXIV, p. 22, 1896.)

magnirostris (V.). (p. 22.),

Australla to Blsmarck Archipelago and north to Borneo and islands of Bay of Bengal

$\begin{array}{ll}\text { Calayan } & \text { Mindanao } \\ \text { Fuga } & \text { Mindoro } \\ \text { Luzon } & \text { Palawan }\end{array}$




\section{Order GRUIFORMES.}

Suborder GRUES.

Family GRUIDF.

ANTIGONE Reichenb.

(Sharpe, Cat. Bds., XXIII, p. 262, 1894.)

sharpei Blanf, Bull. B. O. C., V, p. VI (1895).

Malay Peninsula

Burma and Cochin China

Luzoñ

Suborder PLATALEÆ.

Family IBIDIDAE.

PLEGADIS Kaup.

(Sharpe, Cat. Bds., XXVI, p. 29, 1898.)

falcinellus (Linn.). (1. c.)

China

Afriea

Jamaica

Australia

Eastern United States

Southern Europe to India

Mindanao

\section{Order ARDEHFORMES.}

Suborder CICONIÆE.

Family CICONIIDFE.

Subfamily CICONIINE.

DISSÖURA Cab:

(Sharpe, Cat. Bds., XXVI, p. 294, 1898.)

episcopus (Bodd.). (p. 294.)

Tropical Africa

Indian Peninsula

Malay Peninsula

Indo-Malayan Islands

Indo-Chinese countries

Celebes Ceylon

Calayan Mindoro

Leyte Negros

Luzon Panay

Masbate Samar

Mindanao 
Suborder ARDEZ.

Family ARDEIDFE.

PYRRHERODIAS Finsch \& Hartl., Yog.

Ost-Afr., p. 676 (18\%0).

[Phoyx Stejn. (Sharpe, Cat. Bds., XXVI, p. 60, 1898.)]

manillensis (Meyen). (p. 64.)

\begin{tabular}{ll}
\multicolumn{2}{l}{ Celebes } \\
Indian Peninsula \\
Malay Peninsula \\
Ceylon & \multicolumn{1}{c}{ Cbina } \\
Greater Sunda & Islands \\
\cline { 2 - 2 } & \\
Basilan & Negros \\
Calainianes & Palawan \\
Catanduanes & Panay \\
Cebu & Romblon \\
Leyte & Samar \\
Luzon & Sibuyan \\
Marinduque & Siquijor \\
Masbate & Tablas \\
Mindanao & Tawi Tawi \\
Mindoro & Ticao
\end{tabular}

ARDEA Linn.

(Sharpe, Cat. Bds., XXVI, p. 66, 1898.)

sumatrana Raffl. (p. 68.)

Australia

Malay Peniusula

Sunda Islands Celebes

$\Lambda$ racan

Tenasseriu

Negros

Palawan

cinerea Limn. (p. 74.)

\begin{tabular}{ll} 
Europe & Asia \\
Africa & $\Lambda$ ustraila \\
\hline
\end{tabular}

Guimaras

MESOPHOYX Sharpe.

(Sharpe, Cat. Bds., XXVI, p. 85, 1898.)

intermedia Wagl. (p. 85.)

Indian Peninsula

Malayan Archipelago

Cbina Japan

$\begin{array}{ll}\text { Bobol } & \text { Mindoro } \\ \text { Calayan } & \text { Palawán } \\ \text { Luzon } & \text { Samar } \\ \text { Mindailao } & \end{array}$


HERODIAS Boie.

(Sharpe, Cat. Bds., XXVI, p. 88, 1898.)

timoriensis (cuv.). (p. 98.)

Northern Chlua aud Japan to Australia

Mindanao

EGRETTA Forster.

(Garzella Kanp; Sharpe, Cat. Bds., XXVI, p. 118, 1898.)

garzetta (Limm.). (p. 118.)

Africa
China
$\begin{gathered}\text { Southern } \\
\text { Asia }\end{gathered}$
$\begin{gathered}\text { Indlan Peninsula } \\
\text { Malay Peninsula and Arch } \\
\text { ago }\end{gathered}$
\begin{tabular}{ll} 
Bohol \\
Cagayancillo \\
Leyte & \multicolumn{1}{c}{ Negros } \\
Lubang & Panay \\
Luzon & Romblon \\
Siquijor
\end{tabular}

DEMIEGRETTA Blyth.

(Sharpe, Cat. Bds., XXVI, p. 136, 1898.)

sacra $(G m$.$) . (p. 137.)$

\begin{tabular}{|c|c|}
\hline & Mindanao \\
\hline Bohol & Mindoro \\
\hline Bongao & Negros \\
\hline Cagayancillo & Panay \\
\hline Cagayan Sulu & Pata (off Sulu) \\
\hline Calayan & Romblon \\
\hline Cebu & Semirara \\
\hline Cuyo & Sibuyan \\
\hline Fuga & Siquuljor \\
\hline Lubang & Sulu \\
\hline Luzon & Tablas \\
\hline Malanipa & Tawi Tawi \\
\hline $\begin{array}{l}\text { Marlnduque } \\
\text { Masbate }\end{array}$ & Tícao \\
\hline
\end{tabular}

NYCTIC0RAX Rafin.

(Sharpe, Cat. Bels., XXVI, p. 145, 1898.)

nycticorax (Linn.). (p. 146.)

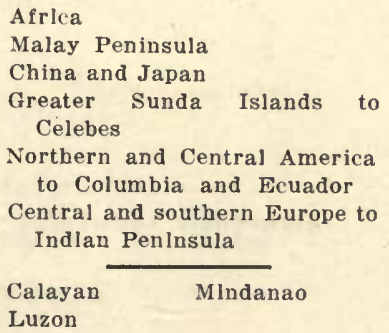


manillensis Vig. (p. 162.)

\begin{tabular}{ll}
$\begin{array}{l}\text { Western } \\
\text { Northern }\end{array}$ \\
\cline { 2 - 2 } Basilan & Morneo \\
Catanduanes & Negros \\
Cebu & Panay \\
Leyte & Samar \\
Luzon & Sibuyan \\
Marinduque & Siquijor \\
Masbate & Tablas \\
Mindanao & Ticao
\end{tabular}

GORSACHIUS $B p$.

(Sharpe, Cat. Bds., XXVI, p. 166, 1898.)

melanolophus (Raff.). (p. 166.)

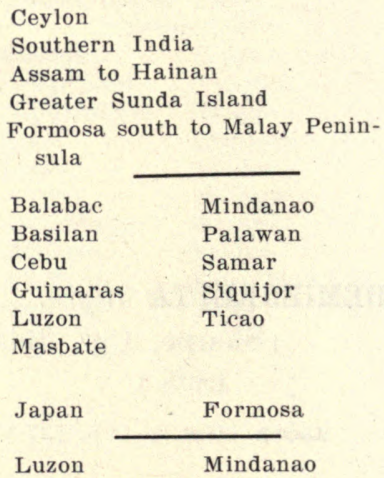

BUTORIDES Blyth.

(Sharpe, Cat. Bds., XXVI, p. 172, 1898.)

javanica (Horsf.). (p. 177.)

Southern China

Malay Peninsula Indian Peninsula

Celebes Ceylon

Mascarene Islands

Greater Sunda Islands

Bongao Mindoro

Cagayancillo Negros

Calamianes Nipa

Calayan Palawan

Cebu Panay

Cuyo - Romblon

Dinagat Samar

Guimaras Semirara

Leyte Sibuyan.

Lubang Siquijor

Luzon Sulu

Marinduque Tablas

Masbate Tawi Tawi

Mindanao Ticao

amurensis (Schrenck) (p. 181.) 
BUBULCUS $B \%$.

(Sharpe, Cat. Bds., XXVI, p. ¿13, 1898.)

coromandus (Bold.). (p. 217.)

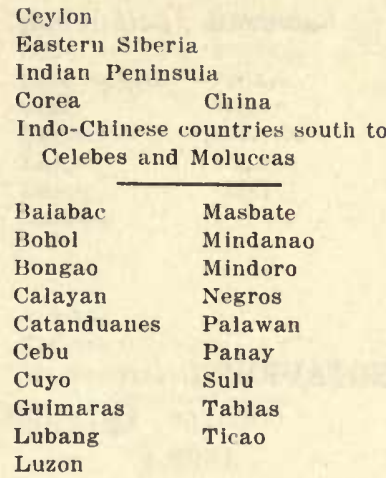

ARDETTA Gray.

(Sharpe, Cat. Bds., XXVI, p. 220, 1898.)

sinensis $(G m$.$) . (p. 227.)$

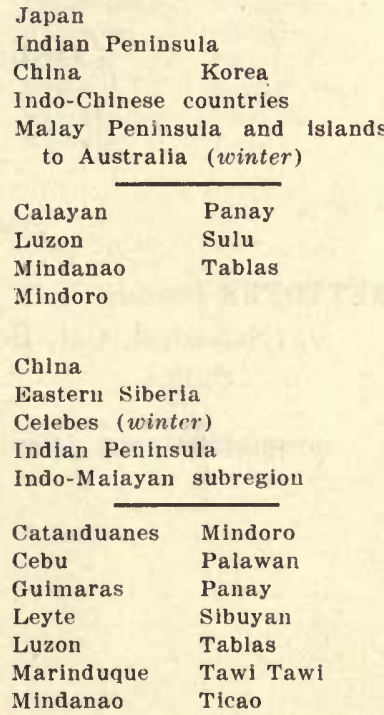

NANNOCNUS Stejn.

(Sharpe, Cat. Bds., XXVI, p. 242, 1898.)

eurythmus (Swinh.). (p. 242.)

\begin{tabular}{ll}
$\begin{array}{l}\text { Eastern } \\
\text { Japan } \\
\text { Borneo }\end{array}$ & $\begin{array}{l}\text { Cblna } \\
\text { Celebes }\end{array}$ \\
\cline { 2 - 2 } Mindanao & Negros
\end{tabular}


DUPETOR Heine \& Reichen.

(Sharpe, Cat. Bds., XXVI, p. 246, 1898.)

flavicollis (Lath.). (p. 247.)

Malay Peninsula
Celebes Ceylon
Indo-Chinese countries
Indian Peninsula
Greater Sunda Islands
Southern and central China
$\begin{aligned} & \text { Cebu Mindanao } \\ & \text { Luzon }\end{aligned}$ Negros
Marinduque $\quad$ Samar

BOTAURUS Briss.

(Sharpe, Cat. Bds., XXVI, p. 253, 1898.)
stellaris (Linn.).
(1. c.)
Northwestern India Burma China
Temperate Palæarctic region
Luzon

\section{Order ANSERIFORMES.}

Family ANATIDF.

Subfamily PLECTROPTERINÆ.

NETTOPUS Brandt.

(Salvadori, Cat. Bds., XXVII, p. 64, 1895.)

coromandelianus $(G m$.$) . (p. 68.)$

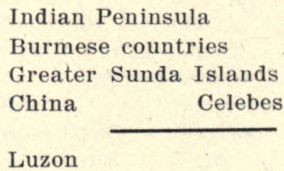

Subfamily ANATIN无.

DENDROCYGNA Swains.

(Salvadori, Cat. Bds., XXVII, p. 144, 1895.)

viduata (Linn.). (p. 145.)

Tropical Africa

Tropical South America

West Indies Madagascar

Luzon 
arcuata (Horsf.). (p. 153.)

guttulata Wallace.

[guttata Forst. (p. 164.)]

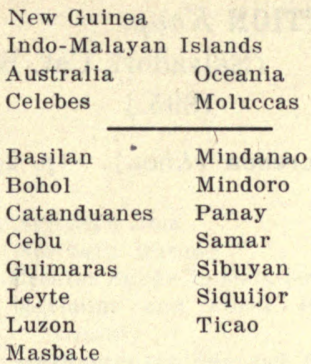

Celebes

Tenimber Islands

Moluccas

New Guinea

Mindanao

$\begin{array}{ll}\text { Catanduanes } & \text { Mindanao } \\ \text { Guimaras } & \text { Mindoro } \\ \text { Lubang } & \text { Panay } \\ \text { Luzon } & \text { Samar } \\ \text { Marinduque } & \text { Siquijor } \\ \text { Masbate } & \text { Ticao }\end{array}$

POLIONETTA Oates.

(Oates, Man. Game Bds. Ind., pt. II, p. 151, 1899.)

[Anas Linn. pt.]

zonorhyncha (Swinh.). (p. 211.)

Eastern Siberia

$\begin{array}{ll}\text { Mongolia } & \text { Japan } \\ \text { China } & \text { Kurile Islands }\end{array}$

Calayan

MARECA Steph.

(Salvadori, Cat. Bds., XXVII, p. 22\%, 1895.)

penelope (Linn.). (l. c.)

\author{
Persia (vinter) \\ Marshall Islands (winter) \\ North America (occasional) \\ Northeastern Africa (winter) \\ Northern Europe and Siberia to \\ Kamtchatka \\ Northern Indian Peninsula and \\ Burma to Borneo (winter) \\ Calayan
}


NETTION Kaup.

(Salvadori, Cat. Bds., XX VII, p. 238, 1895.)

crecca (Linn.). (p. 243.)

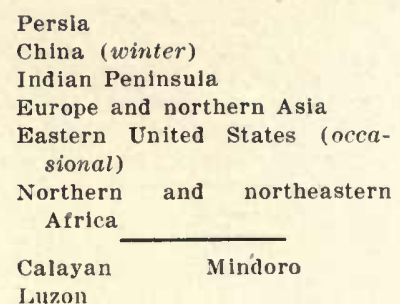

DAFILA Steph.

(Salvadori, Cat. Bds., XXVII, p. 280, 1895.)

acuta (Linn.). (p. 270.)

[modesta Tristr. (p. 275, note.)]

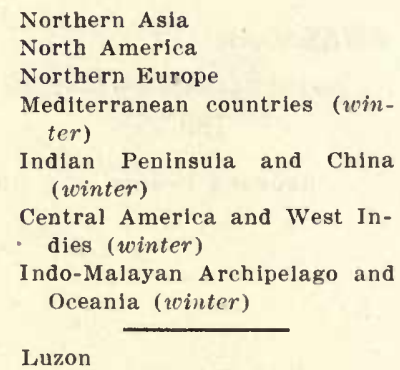

QUERQUEDULA Steph.

(Salvadori, Cat. Bils., XXYII, p. 290 , 1895.)

querquedula (Linn.).

[circia Jinn. (p. 293.)]

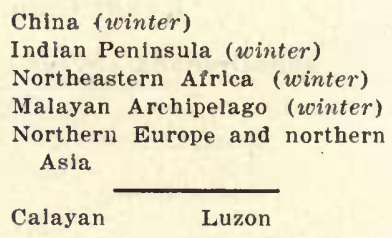

SPATULA Boie.

(Salvadori, Cat. Bds., XXVII, p. 306, 1895.)

clypeata (tinn.). (p. 306.)

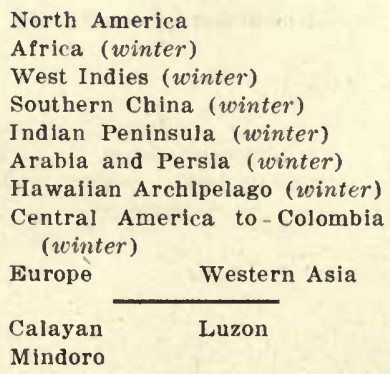


FULIGULA Steph.

(Salvadori, Cat. Bds., XXYII, p. 354, 1895.)

fuligula.(Lim.). (p. 363.)

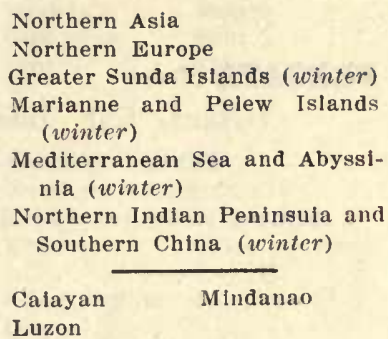

\section{Order PELECANIFORMES.}

Family PHALACROCORACIDAE.

PHALACROCORAX Briss.

(Grant, Cat. Bds., XXVI, p. 330 , 1898.)

carbo (Linn.). (p. 340.)

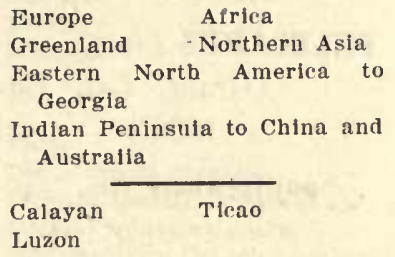

Family PLOTIDAE.

ANHINGA Briss.

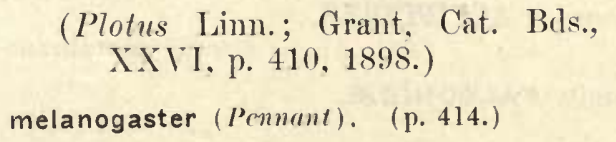

Mesopotamia

Indian Peninsula

Indo-Chinese countries

Indo-Malayan Peninsula

Borneo

Celebes

Luzon

Mindanao Negros

Family SULIDAE.

SULA Briss.

(Grant, Cat. Bds., XXVI, p. 423, 1898.)

piscator (Jimn.). (p. 432.)

Oceanla

Southern Atlantlc

Indian and Australian Seas

Mindanao 
sula (Linn.). (p. 436.)
Tropical Atlantic, Indian, Australian, and Pacific Oceans

Calayan ? $^{1}$ Luzon

Dinagat Mindanao

Family FREGATIDF.

FREGATA Briss.

(Grant, Cat. Bds., XXVI, p. 442, 1898.)

aquila (Linn.). (p. 443.)

Tropical and subtropical oceans of both hemispheres

Cagayancillo

ariel (Gould). (p. 447.)

Tropical and subtropical Indian and Pacific Oceans

Cagayancillo N.gros

Luzon Palawan

Mindanao

Family PELECANIDAE.

PELECANUS Linn.

(Grant, Cat. Bds., XXVI, p. 460, 1898.)

philippensis $6 m$. (p. 471.)

Indian Peninsula to China

Luzon Mindanao

\section{Order ACCIPITRIFORMES.}

\section{Suborder ACCIPITRES.}

\section{Family FALCONIDAE.}

Subfamily ACCIPITRINÆ.

\section{CIRCUS Lacép.}

(Sharpe, Cat. Bds., I, p. 50, 18\%4.)

spilonotus Kaup. (p. 58.)

Eastern Siberia
China and Indo-Burmese coun-
tries (winter)
Malay Archipelago (winter)
$\begin{array}{ll}\text { Calayan } & \text { Palawan } \\ \text { Luzon } & \text { Sibay } \\ \text { Mindanao } & \text { Sulu } \\ \text { Mindoro } & \end{array}$

${ }^{1}$ A pair of birds, in all probability S. sula, were observed near Calayan by $\mathrm{Mr}$. MeGregor in September, 1903.-Worcester. 
melanoleucus (Forst.). (p. 61.)

æruginosus (Linn.). (p. 69.)
Eastern Siberia

Mongolia China (winter)

Northeastern Indian Peninsula and Indo-Chinese countries (winter)

$\begin{array}{ll}\text { Calayan } & \text { Mindanao } \\ \text { Basilan } & \text { Negros } \\ \text { Guimaras } & \text { Sulu } \\ \text { Luzon } & \text { Ticao }\end{array}$

Temperate Europe

Northern Africa (winter)

Indian Peninsula (winter)

Siberia China (winter)

Cagayan Sulu Mindoro

Luzon
ASTUR Lacép.

(Sharpe, Cat. Bds., I, p. 92, 1874.)

trivirgatus (Temm.). (p. 105.)

soloensis (Lath.). (p. 114.)

cuculoides (Temm.). (p. 115.)

\begin{tabular}{ll}
$\begin{array}{l}\text { Indian Peninsula } \\
\text { Indo-Malayan } \\
\text { Ceylon }\end{array}$ & $\begin{array}{l}\text { Islands } \\
\text { Formosa }\end{array}$ \\
\cline { 2 - 2 } $\begin{array}{l}\text { Balabac } \\
\text { Leyte } \\
\text { Mindanao }\end{array}$ & $\begin{array}{l}\text { Palawan } \\
\text { Samar }\end{array}$
\end{tabular}

China

Indo-Chinese countries Molucea Islands (winter) Malay Peninsula (winter) Indo-Malayan Islands (winter)

Cagayancillo Mindanao Luzon

China, south to Malay Archipelago (winter)

Cagayancillo

ACCIPITER Briss.

(Sharpe, Cat. Bds., I, p. 132, 18\%4.) gularis (Temm. \& Schl.). (p. 160, pt.)

Japan

Northern China

Malay Peninsula

Malay Archipelago

Calayan Palawan

Fuga

manillensis (Meyen). (p. 150, pt.)

$\begin{array}{ll}\text { Guimaras } & \text { Mindanao } \\ \text { Leyte } & \text { Mindoro } \\ \text { Luzon } & \text { Negros }\end{array}$


Subfamily AQUILIN 死.

LOPHOTRIORCHIS Sharpe.

(Sharpe, Cat. Bds., I, p. 255, 18\%4.)

kieneri (Geoffr.). (p. 255.)

Malay Peninsula
Indian Peninsula
Lesser Sunda Islands
$\begin{aligned} & \text { Indo-Malayan Islands } \\ & \text { Indo-Chinese provinces } \\ & \text { Ceylon }\end{aligned}$
$\begin{array}{ll}\text { Luzon } \\ \text { Panay }\end{array}$

SPIZA ĖTUS Vieill.

(Sharpe, Cat. Bds., I, p. 259, 18\%4.)

philippinensis Gurney. (p. 261, note.)

$\begin{array}{ll}\text { Basilan } & \text { Negros } \\ \text { Luzon } & \text { Palawan } \\ \text { Masbate } & \text { Siquijor }\end{array}$

limnaëtus (Horsf.). (p. 272.)

Burmese provinces
Malayan Peninsula
Northeastern Bengal
Greater Sunda
$\begin{aligned} & \text { Assam } \\ & \text { Islands } \\ & \text { Calamianes }\end{aligned}$
$\begin{array}{ll}\text { Lubang } \\ \text { Mindanalayas }\end{array}$

PITHECOPHAGA Grant.

(Grant, B. O. C., VI, p. 17, 1896.)

jefferyi Grant, Bull. B. O. C., VI, p. 17 , 1896 ; id. Ibis, 1897 , pl. v.

Leyte ? ${ }^{1}$ Mindanao

Luzon Samar

SPILORNIS Gray.

(Sharpe, Cat. Bds., I, p. 28\%, 1874.)

bacha (Daud.). (p. 290.)

Malayan Peninsula

Greater Sunda Islands

Calamianes Palawan

Balabac

holospilus (Vig.). (p. 293.)

$\begin{array}{ll}\text { Basilan } & \text { Marinduque } \\ \text { Bongao } & \text { Mindanao } \\ \text { Catanduanes } & \text { Mindoro } \\ \text { Cebu } & \text { Samar } \\ \text { Leyte } & \text { Sulu } \\ \text { Luzon } & \text { Tawi Tawi }\end{array}$

panayensis Steere, List. Bds. \& Mams. Steere Exp., p. 7 (1890).

$\begin{array}{ll}\text { Guimaras } & \text { Romblon } \\ \text { Masbate } & \text { Sibuyan } \\ \text { Negros } & \text { Tablas } \\ \text { Panay } & \end{array}$

${ }^{1} P$. jefferyi was probably heard, although not seen, by Whitehead in Leyte. As it has been killed in Samar and Mindanao its occurrence in Leyte is hardly open to doubt.-WORCESTER. 
BUTASTUR Hodgs.

(Sharpe, Cat. Bds., I, p. 294, 1874.)

indicus $(G m$.$) (p. 297.)$

\begin{tabular}{ll}
$\begin{array}{l}\text { Ussurl-land } \\
\text { Japan } \\
\text { China to Malay }\end{array}$ & $\begin{array}{l}\text { Celebes } \\
\text { Balabac }\end{array}$ \\
\cline { 2 - 2 } Basllan & Masbate \\
Bohol & Mindanao \\
Cagayanclllo & Nindoro \\
Cagayan Sulu & Palawan \\
Calamlanes & Panay \\
Calayan & Samar \\
Cebu & Slbay \\
Cuyo & Siquijor \\
Fuga & Sulu \\
Gulmaras & Tawi Tawi \\
Lubang & Verde \\
Luzon &
\end{tabular}

HALIAËTUS Savign.

(Sharpe, Cat. Bds., I, p. 301, 1874.)

leucogaster $(G m$.$) . (p. 307.)$

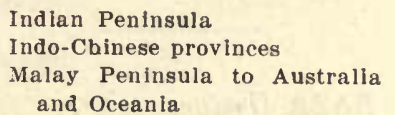

Basllan Romblon

Bongao Samar

Calamianes Senilrara

Calayan Sibuyan

East Bolod Slqulfor

Fuga Sulu

Luzon Tablas

Masbate Tawi Tawl

Mindanao Ticao

MIndoro Verde

Palawan

HALIASTUR Selby.

(Sharpe, Cat. Bds., I, p. 312, 1874.)

intermedius (iurney. (p. 314.)

\begin{tabular}{ll} 
Celebes \\
Malay Peninsula \\
Greater Sunda & Islands \\
Indo-Chinese provinces \\
\cline { 2 - 2 } Basllan & \multicolumn{1}{l}{ Marinduque } \\
Bohol & Masbate \\
Bongao & Mindanao \\
Calamianes & Mindoro \\
Catanduanes & Negros \\
Cebu & Panay \\
Cuyo & Romblon \\
Guimaras & Samar \\
Leyte & Slbuyan \\
Lubang & Siquijor \\
Luzon & Sulu \\
Maestro de & Tablas \\
Campo & Tawl Tawi \\
Malanlpa & Ticao
\end{tabular}


ELANUS Savign.

(Sharpe, Cat. Bds., I, p. 336, 1874.)

hypoleucus Gould. (p. 338.)

\begin{tabular}{|c|c|c|}
\hline $\begin{array}{l}\text { Celebes } \\
\text { Greater }\end{array}$ & Sunda & Islands \\
\hline Basilan & & Mindoro \\
\hline Bongao & & Negros \\
\hline Calamia & nes & Samar \\
\hline Cebu & & Sibuyan \\
\hline Guimara & & Sulu \\
\hline Luzon & & Tawi Taw \\
\hline
\end{tabular}

PERNIS Cuv.

(Sharpe, Cat. Bds., I, p. 343, 18\%4.)

ptilonorhynchus (Temm.). (p. 347.)

\begin{tabular}{|c|c|c|}
\hline \multicolumn{3}{|c|}{$\begin{array}{l}\text { Ceylon } \\
\text { Malay Peninsula } \\
\text { Indian Peninsula } \\
\text { Indo-Chinese countries } \\
\text { Greater Sunda Islands }\end{array}$} \\
\hline Cebu & & Palawan \\
\hline Luzon & & Samar \\
\hline Mindana & & Sibuyan \\
\hline Mindoro & & Sulu \\
\hline
\end{tabular}

BAZA Hodge.

(Sharpe, Cat. Bds., I, p. 351, 18\%4.)

magnirostris Gray. (p. 356.)

Luzon Mindanas

leucopais Sharpe, Ibis, 1888, p. 195.

Palawan Samar

Romblon

Samar

MICROHIERAX Sharpe.

(Sharpe, Cat. Bds., I, p. 366, 18\%4.)

erythrogenys (Vig.). (p. 369.)

Luzon Mindoro

meridionalis Grant, Ibis, 1897, 220.

$\begin{array}{ll}\text { Cebu } & \text { Mindanao } \\ \text { Luzon } & \text { Samar }\end{array}$

\section{FALC0 Linn.}

(Sharpe, Cat. Bds., I, p. 3\%4, 18\%4.)

peregrinus Tunst.

[communis Gm. (p. 376.)] ernesti Sharpe, Ibis, 1894, p. 545.

\author{
Africa (winter) \\ Northern America \\ Europe Asia \\ Indian Peninsula (winter) \\ Calayan Mindoro \\ Catanduanes Negros \\ Fuga Palawan \\ Luzon Sibuyan \\ Mindanao \\ Greater Sunda Islands \\ New Guinea Fiji Islands \\ Luzon \\ Negros
}


melanogenys Gould. (p. 385.)

severus Horsf. (p. 397.)
Australia

\section{Luzon}

Malay Peninsula
Burmese provinces
Himalayas Ceylon
Southern Indian Peninsula
Greater Sunda Islands to New
Guinea and New Britain
$\begin{array}{ll}\text { Calamianes } & \text { Palawan } \\ \text { Cebu } & \text { Romblon } \\ \text { Luzon } & \text { Sibuyan } \\ \text { Mindanao } & \text { Siquijor } \\ \text { Mindoro } & \text { Sulu } \\ \text { Negros } & \text { Tawi Tawi }\end{array}$

CERCHNEIS Boie.

(Sharpe, Cat. Bds., I, p. 423, 1874.)

tinnunculus (Linn.). (p. 425.)

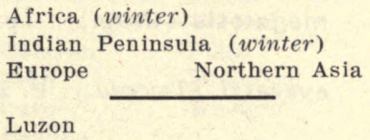

Suborder PANDIONES.

Family PANDIONIDAE.

PANDION Savign.

(Sharpe, Cat. Bds., I, p. 448, 1874.)

haliaëtus (Linn.). (p. 449.)

Eastern Hemisphere

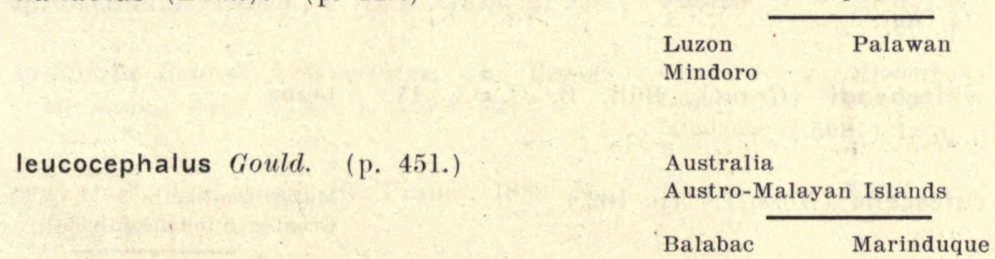

\section{POLIOAËTUS Kaup.}

(Sharpe, Cat. Bds., I. p. 452, 18\%4.)

ichthyaëtus (Horsf.). (p. 452.)

Malay Peninsula

Burmese provinces

Indian Peninsula

Greater Sunda Islands

Celebes Ceylon

Basilan

Bongao

Mindoro

Luzon

Negros

Mindanao Samar 


\section{Order STRIGINORMIES.}

\section{Family BUBONIDE.}

Subfamiiy BUBONINE.

PÁEUDOPTYNX Kimu.

(Bubo, pt., Sharpe, Cat. Bels., 11, p. $12,18 \% 5$.

$\begin{array}{ll}\text { philippinensis K(йp. (1. 43.) } & \text { Luzon } \\ \text { gurneyi Twocdd., P. \%. S., 1878, p. } 940 . & \text { Mindaua }\end{array}$

\section{OTUS Pennant.}

(Stone, Auk. XX, p. 275, 1903.)

(Scops. Sharpe, Cat. Bds., 1I, p. 43, 1875.)

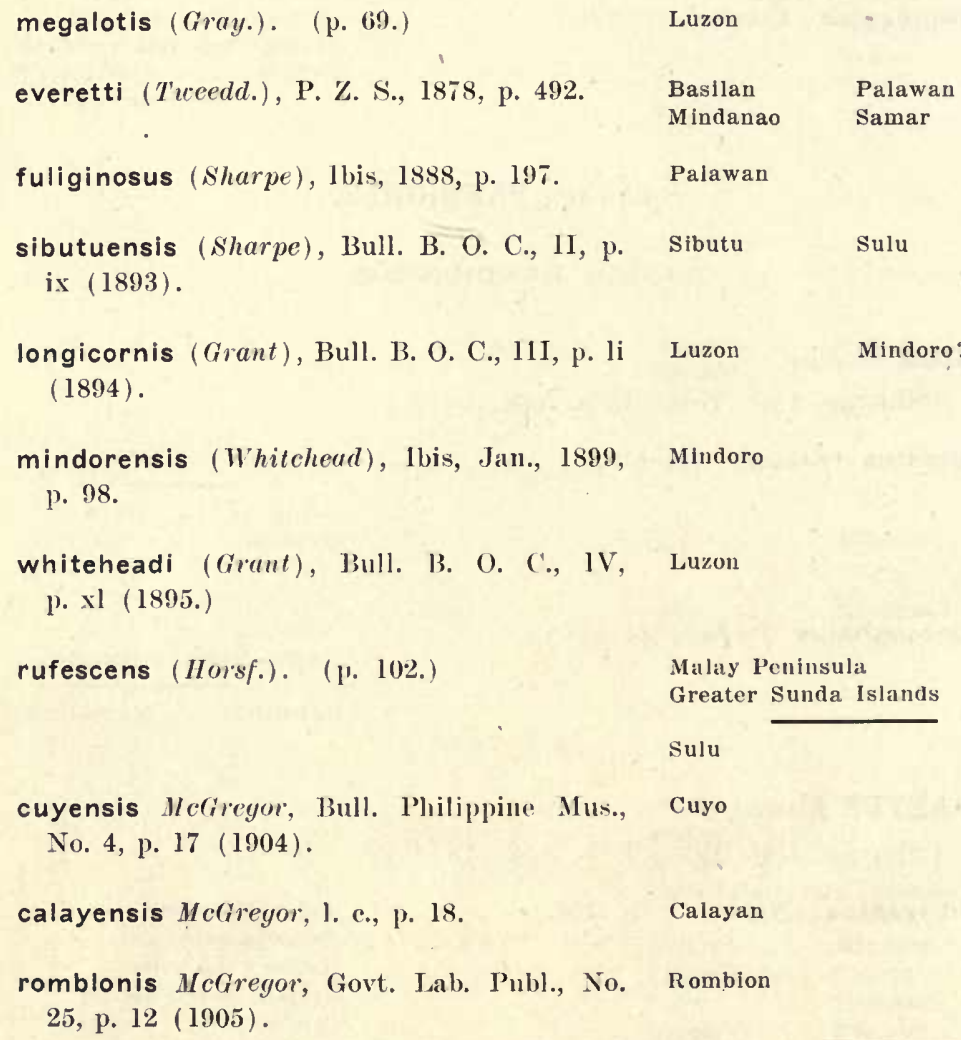

${ }^{1}$ An undetermined specimen of Scops collected by Whitehead in Mindoro, is doubtfully referred by Grant to $s$. Iongicornis (Ibis, Oct., 1896, p. 462).WORCESTER. 
NINOX $/ 1$ odgs.

(Sharpe, Cat. Bds., II, p. 151, 1875.)

lugubris (Tickell). (p. 154.)

Himalayas

Indian Peninsula

Burmese countries

$\begin{array}{ll}\begin{array}{ll}\text { Baslian } \\ \text { Luzon }\end{array} & \begin{array}{l}\text { Negros } \\ \text { Masbate }\end{array} \\ \text { Mindanao } & \text { Sulu } \\ \text { Soutbern India } \\ \text { Malay Peninsuia } \\ \text { Greater Sunda }\end{array}$

japonica (Temm. (f Schl.).

(Scutulata pt. Sharpe, Cat. Bds., II, p. 156 ; florensis Wall., P. Z. S., 1863, p. 488; macroptera W. Blasius, Ornis, 1888 , p. 545.)

philippensis $B p . \quad(1.16 \overline{7}$.

everetti Sharpe, Bull. B. O. C., V1, p. 42

Siasi

$(1897)$.

spilocephala T'weedd., P. Z. S., 1878, p. 940.

Basllan

Mindanao

spilonota Bourns \& Worcester, Oce. Papers

Mimnesota Acad., I, No. 1, p. 8 (1894).

Cebu

Mindoro

Luzon

Tabias

Mindanao

reyi Oust., Bul. Assoc. Sc. France, 1880, No.

Bonguo

Sulu

39, p. 206.

mindorensis Grant, Ibis, 1896, p. 463.

Mindoro

plateni Blas. (in litt.) Hartl., Abl. Nat. Mindoro Ver. Brem., XVI, p. 27 I (1899).

Subfamily SYRNIINÆ.

\section{SYRNIUM Savign.}

(Sharpe, Cat. Bds., II, p. 244, 1875.)

whiteheadi Sharpe, Ibis, 1888, p. 196, pl. iii.

Palawan

[uiepleni W. Blasius, Ornis, 1888, p. 304.] 
Family STRIGIDÆ.

STRIX $\operatorname{Linn}$.

(Sharpe, Cat. Bds., II, p. 290, 1875.)

candida Tick. (p. 308.)

[oustaleti Hartl., P. Z. S., 1879, p. 295.]

Indian Peninsula
Burmese provinces
Northern Australia
Fiji Islands (Viti Levu)
Formosa

Order PSITTACIFORMES.

Family LORIIDAE.

TRICHOGLOSSUS Vig. \& Horsf.

(Salvad., Cat. Bds., XX, p. 49, 1891.)

johnstoniæ Hartert, Bull. B. O. C., XIV, Mindanao p. 10 (1903).

\section{Family CACATUIDF.}

CACATUA Vieill.

(Salvadori, Cat. Bds., XX, p. 115, 1891.)

\begin{tabular}{|c|c|c|c|}
\hline hæmaturopygia & (P. L. S. Müll.). (p. 130.) & $\begin{array}{l}\text { Balabac } \\
\text { Basilan } \\
\text { Bongao } \\
\text { Calamianes } \\
\text { Cebu } \\
\text { Guimaras } \\
\text { Lapac } \\
\text { Leyte } \\
\text { Lubang } \\
\text { Luzon } \\
\text { Marinduque } \\
\text { Masbate } \\
\text { Mindanao }\end{array}$ & $\begin{array}{l}\text { Mindoro } \\
\text { Negros } \\
\text { Nipa } \\
\text { Palawan } \\
\text { Panaon } \\
\text { Panay } \\
\text { Samar } \\
\text { Siquijor } \\
\text { Sulu } \\
\text { Tablas } \\
\text { Tawi Tawi } \\
\text { Ticao }\end{array}$ \\
\hline & Family PSITTACID & & \\
\hline
\end{tabular}

PRIONITURUS Wagl.

(Salvadori, Cat. Bds., XX, p. 414, 1891.)
verticalis Sharpe, Bull. B. O. C., III, p. x (1893).
Bongao
Sibutu
Tawi Tawi
montanus Grant, Bull. B. O. C., IV, p. xli Luzon $(1895)$. 
discurus (Vicill.). (1. 417.)

$\begin{array}{ll}\text { Basilan } & \text { Mindanao } \\ \text { Catanduanes } & \text { Negros } \\ \text { Cebu } & \text { Samar } \\ \text { Guimaras } & \text { Sibuyan } \\ \text { Leyte } & \text { Suiu } 1 \\ \text { Luzon } & \text { Tabias }\end{array}$

[P. sulucusis W. Blasius, J. f. O. 1890 , p. 140.$]$

Masbate

waterstradti lioths., Bull. B. O. C., XIV, Mindanao p. $72(1904)$.

mindorensis Stecre. (p. 419.)

Mindoro

cyaneiceps Sharpe. (p. 419.)

Balabac Palawan

Calamianes

luconensis steere. (p. 420.)

Luzon

Marinduque

TANYGNATHUS Wagl.

(Salvadori, Cat. Bds., XX, p. 422 . 1891.)

lucionensis (Linn.). (p. 424.)

\begin{tabular}{|c|c|}
\hline Basilan & Mindoro \\
\hline Bongao & Negros \\
\hline Cagayan Suiu & Palawan \\
\hline Calamianes & Panay \\
\hline Cebu & Romblon \\
\hline GuImaras & Samar \\
\hline Lapac & Sibutu \\
\hline Leyte & Sibuyan \\
\hline Luzon & Siquijor \\
\hline Maestro de & Sulu \\
\hline Campo & Tablas \\
\hline Malanipa & Tawl Taw \\
\hline Marinduque & Ticao \\
\hline Masbate & Verde \\
\hline Mindanao & \\
\hline Luzon & Negros \\
\hline Mindanao & Panay \\
\hline Mindoro & Samar \\
\hline & Tawi Taw \\
\hline & \\
\hline
\end{tabular}

burbidgei Sharpc. (p. 432.) Bongao Tawi Tawi

BOLBOPSITTACUS Salvad.

(Salvadori, Cat. Bds., XX, p. 503, 1891.)

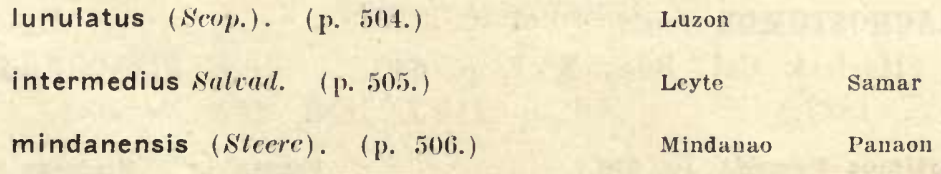

${ }^{1}$ W. Blasius has separated the Sulu birds as a new variety under the name $P$. discurus suluensis, stating that they differ from the typical $P$. discurus in the greater extent of the blue coloring on the head and in the more slender and longer beak. A careful examination of the speeimens obtained by Bourns and myself in Sulu failed to disclose any differences between them and typical $P$. discurus, and 1 am of the opinion that Blasius's "variety" can not stand.WORCESTER.

$35916-4$ 
LORICULUS Blyth.

(Salvadori, Cat. Bds., XX, p. 515, 1891.)

\begin{tabular}{|c|c|c|}
\hline chrysonotus Scl. (p. 522.) & Cebu & \\
\hline regulus Souancé. (p. 523.) & $\begin{array}{l}\text { Guimaras } \\
\text { Masbate } \\
\text { Negros }\end{array}$ & $\begin{array}{l}\text { Panay } \\
\text { Ticao }\end{array}$ \\
\hline $\begin{array}{l}\text { bournsi McGregor, Govt. Lab. Publ. No. 25, } \\
\text { p. } 16 .\end{array}$ & $\begin{array}{l}\text { Romblon } \\
\text { Sibuyan }\end{array}$ & Tablas \\
\hline philippensis (P. L. S. Müll.). (p. 524.) & $\begin{array}{l}\text { Catanduanes } \\
\text { Luzon }\end{array}$ & Marindu \\
\hline mindorensis Steere. (p. 526.) & Mindoro & \\
\hline siquijorensis Steere. (p. 526.) & Siquijor & \\
\hline apicalis Souancé. (p. 528.) & $\begin{array}{l}\text { Basilan } \\
\text { Bazol }\end{array}$ & $\begin{array}{l}\text { Dinigat } \\
\text { Mindanao }\end{array}$ \\
\hline worcesteri Steere. (p. 528, pt.) & $\begin{array}{l}\text { Bohol } \\
\text { Leyte }\end{array}$ & Samar \\
\hline bonapartei Souancé. (p. 530.) & $\begin{array}{l}\text { Bongao } \\
\text { Sulu }\end{array}$ & Tawi Ta \\
\hline sp. Salvad., Cat. Bds. XX, p. 530, note. & Mindanao & \\
\hline galgulus (Linn.). (p. 531.) & $\begin{array}{l}\text { Malay Penins } \\
\text { Sumatra } \\
\text { Banka }\end{array}$ & $\begin{array}{l}\text { Nias } \\
\text { Borneo }\end{array}$ \\
\hline
\end{tabular}

\section{Order CORACIIFORMES.}

Suborder PODARGI.

Family PODARGIDAE.

\section{BATRACHOSTOMUS Gould.}

(Hartert, Cat. Bds., XVI, p. 636, 1892.)

septimus Tweedd. (p. 638.) Basilan ${ }^{1}$ Mindanao

microrhynchus Grant, Bull. B. O. C., IV, Luzon p. xli (1895.)

${ }^{1}$ A Batrachostomus, probably of this species, was seen by me in Basilan in 1891.-WORCESTER. 
menagei Bourns of Worcester, Occ. Papers Minnesota Acad., I, No. 1, p. 11 (1894).

javensis (Horsf.). (p. 640.)

Negros? $1 \quad$ Panay

Borneo Sumatra

Java

Palawan

affinis Blyth. (p. 643.)

Malay Peninsula

Southern Tenasserin

Borneo

Sumatra

Palawan

Suborder CORACI

Family CORACIIDAE.

Subfamily CORACIIN

EURYSTOMUS Vieill.

(Sharpe, , Cat. Bds., XVII, p. 28, 1892.)

orientalis (Linn.). (p. 33.)

\begin{tabular}{ll}
\multicolumn{2}{l}{ Malay Peninsula } \\
Burmese provlnees \\
Cachar & \multicolumn{1}{l}{ Java } \\
Borneo & \multicolumn{1}{c}{ Sumatra } \\
Andaman and & Nicobar Islands \\
\cline { 2 - 2 } Basilan & Mindoro \\
Cagayancillo & Negros \\
Calamianes & Palawan \\
Calayan & Panaon \\
Cebu & Panay \\
Cuyo & Romblon \\
Dinagat & Samar \\
Fuga & Stbutu \\
Guimaras & Sibuyan \\
Leyte & Siquijor \\
Luzon & Sulu \\
Maestro de & Tablas \\
\multicolumn{1}{c}{ Campo } & Tawl Tawi \\
Marinduque & Ticao \\
Masbate & Verde \\
Mindanao &
\end{tabular}

Suborder HALCYONES.

Family ALCEDINIDA.

Subfamily ALCEDININ正.

PELARGOPSIS Giloger.

(Sharpe, Cat. Bds., XVII, p. 96, 1892.)

leucocephala $(G m.) .^{2} \quad$ (p. 98.)

Borneo

Palawan?

${ }^{1}$ A female Batrachostomus, probably referable to this species, was shot in Negros by Keay in April, 1899 (See Ibis, April, 1900, p. 353.)-Worcester.

'The Palawan record is based on Whitehead's collection; Sharpe, Ibis, 1888, p. 197; Whitehead records $P$. gouldi not $P$. leucocephala, Ibis, 1893, p. 45.-McGregor. 
gouldi Sharpe. (p. 100.)

gigantea Walden. (p. 100.)

$\begin{array}{ll}\text { Balabac } & \text { Luzon } \\ \text { Calamianes } & \text { Mindoro } \\ \text { Lubang } & \text { Palawan }\end{array}$

$\begin{array}{ll}\text { Basilan } & \text { Negros } \\ \text { Bongao } & \text { Panay } \\ \text { Cebu } & \text { Samar } \\ \text { Dinagat } & \text { Sibutu } \\ \text { Guimaras } & \text { Sibuyan } \\ \text { Lapac } & \text { Sulu } \\ \text { Leyte } & \text { Tablas } \\ \text { Masbate } & \text { Tawi Tawi } \\ \text { Mindanao } & \text { Ticao } \\ \text { Malanipa } & \end{array}$

ALCED0 Linn.

(Sharpe, Cat. Bds., XVII, p. 141, 1892.)

bengalensis Briss. (p. 141.)

meninting Horsf. (p. 157.)

\begin{tabular}{ll} 
Molucca Islands \\
Malay Peninsula \\
Indian Peninsula \\
Burmese provinces \\
Greater Sunda & Islands \\
China & \multicolumn{1}{l}{ Hainan } \\
Europe & Northern Asia \\
\cline { 2 - 2 } Balabac & Masbate \\
Basilan & Mindanao \\
Bohol & Mindoro \\
Cagayancillo & Negros \\
Cagayan Sulu & Palawan \\
Calamianes & Panay \\
Calayan & Romblon \\
Catanduanes & Samar \\
Cebu & Sibuyan \\
Cuyo & Siquijor \\
Guimaras & Sulu \\
Leyte & Tablas \\
Lubang & Tawi Tawi \\
Luzon & Verde
\end{tabular}

Sumatra

Malay Peninsula

Southern Tenasserim

\begin{tabular}{ll} 
Java & Borneo \\
\cline { 2 - 2 } Balabac & Palawan \\
Bongao & Sulu \\
Calamianes & Tawi Tawi
\end{tabular}

ALCYONE Swains.

(Sliarpe, Cat. Bds., XVII, p. $16 \%$, 1892.)

cyanopectus (Lafr.) (p. 185.)

[steerii (Sharpe). (p. 187.)]

$\begin{array}{ll}\text { Luzon } & \text { Mindoro } \\ \text { Marinduque } & \text { Sibuyan } \\ \text { Masbate } & \text { Ticao }\end{array}$

argentata (Tweedd.). (p. 187.)
Mindanao
Basilan Dinagat 
flumenicola (steere). (p. 187.)

Leyte

Samar

nigrirostris (Bourns of Worcester), Occ. Pa-

Cebu

pers Minncsota Acad., I, No. 1, p. 13 Negros

Panay

(1894).

Subfunily DACELONINE.

CEYX Lacép.

(Sharpe, Cat. Bils., XVII, p. 173, 1892.)

melanura Kump. (p. 180.)

Isizon

mindanensis Steere. (1). 181, note.)

Basilan Mindanao

[busilanica Streere. (p. 181.)]

euerythra Sharpe. (p. 179.)

Malay Peninsula

Sumatra

Borneo

Balabac

Palawan

Bongao

Sulu ??

Calamianes Tawl Taw

Mindoro

bournsi Stecre. (p. 185.)

[malamani Steere. ${ }^{3}$ (p. 184.)]

$\begin{array}{ll}\text { Basilan } & \text { Romblou } \\ \text { Bongao } & \text { Sibuyan } \\ \text { Cebu } & \text { Siquijor } \\ \text { Malamaui } & \text { Sulu } \\ \text { Mindanao } & \text { Tabias } \\ \text { Negros } & \text { Tawi Tawi }\end{array}$

samarensis Stecre, List Bds. \& Mams. Steere

Leyte

Samar

Exp., p. $10(1890)$.

${ }^{1}$ I have already recorded my opinion elsewhere that $C$. basilawica Steerc is a synonym for $C$. mindanensis (Occ. Papers Minnesota Acad., No. 1, p. 457).WORCESTER.

${ }^{2}$ Ceyx eucrythra is recorded by Sharpe, Hand-List of Birds, p. 353, from the Sulu Islands. This species has been obtained on Bongao and Tawi Tawi, but so far as I can learn has not yet been met with on Sulu.-Worcester.

${ }^{3}$ As a result of the examination of a series of sixty-six specimens, Bourns and I reached the conclusion that $C$. malamani Steere was a synonym of $C$. bournsi Steere. From the specimens collected by us it was possible to select a practically unbroken series between a bird with a magnificent deep-blue upper surface and a bird with a silvery white upper surface which had not a blue feather on it [Oce. Paper's Mimesota Acad., I, No. 1, p. 47 (1894)].-Worcester. 
HALCYON Swains.

(Sharpe, Cat. Bds., XVII, p. 213, 1892.)

coromandus (Lath.). (p. 217.)

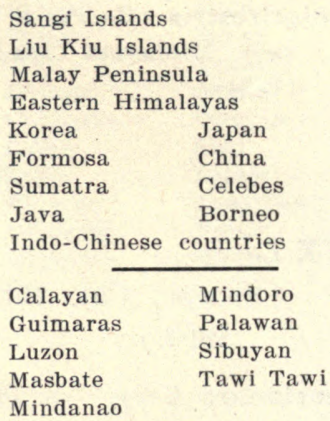

gularis $(K u h l) . \quad$ (p. 227.)

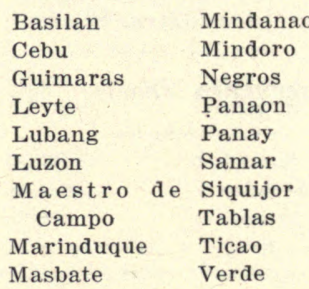

pileatus (Bodd.). (p. 229.)

Ceylon

Malay Peninsula

Southern India to Canara

Java Borneo

Sumatra Celebes

Indo-Chinese countries to China and north to Korea

$\begin{array}{ll}\text { Balabac } & \text { Palawan } \\ \text { Basilan } & \text { Tawi Tawi }\end{array}$

winchelli Sharpe. (p. 255.)

$\begin{array}{ll}\text { Basilan } & \text { Samar } \\ \text { Bongao } & \text { Sibuyan } \\ \text { Cebu } & \text { Siquijor } \\ \text { Mindanae } & \text { Sulu } \\ \text { Negros } & \text { Tablas } \\ \text { Panay } & \text { Tawi Tawi } \\ \text { Romblon } & \end{array}$


chloris (Bodd.). (p. 273.)

hombroni (Bp.). (p. 284.)

lindsayi (Vig.). (p. 286.)

moseleyi Steere. (p. 288.)

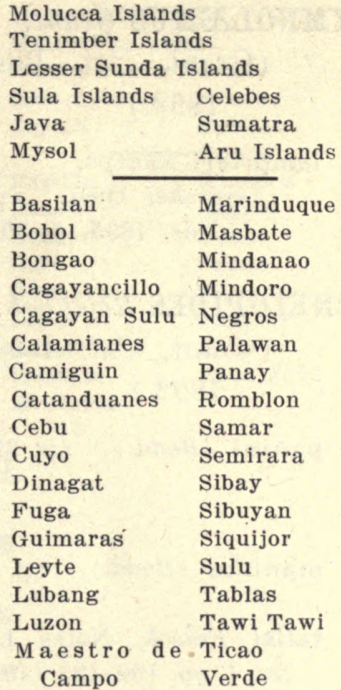

Mindanao

Luzon

Negros

Suborder BUCER0TES.

Family BUCEROTIDF.

HYDROCORAX Briss.

(Grant, Cat. Buds., XVII, p. 35\%, 1892.)

hydrocorax (Linn.). (p. 358.)

Luzon Marinduque

mindanensis (Tweedd.). (p. 359.)

Basilan

Mindanao

semigaleatus (Tweedd.). (p. 360.)

Leyte

Samar

Panaon

ANTHRACOCEROS Reichenb.

(Grant, Cat. Bds., XVII, p. 361, 1892.)

montani (Oust.). (p. 370.)

Sulu

Tawi Tawi 
GYMNOLAEMUS Grant.

(Grant, Cat. Bds., XVII, p. 370, 1892.)

lemprieri Sharpe.

[marchei Oust. (p. 370.) Cf. Everett,

Balabac

Palawan Ibis, 1895 , p. 30.$]$

PENELOPIDES Reichenb.

(Grant, Cat. Bds., XVIII, p. 3r1, 1892.)

panini (Bodd.). (p. 372.)

manillæ (Bodd.). (p. 373.)

Guimaras Panay

Masbate Ticao

Negros

Luzon Marinduque

talisi Finsch, Notes Leyden Mus., XXIII,

Luzon (Cagayan)

No. 4, pp. 190-193 (1903).

mindorensis Steere. (p. 374.)

Mindoro

affinis Tweedd. (p. 375.)

Dinagat

Mindanao

basilanica Steere. (p. 375.)

Basilan

samarensis Steere. (p. 376.)

Leyte

Samar

CRANORRHINUS Cab. \& Heine.

(Grant, Cat. Bds., XVII, p. 3\%\%, 1892.)

leucocephalus (Vieill.). (p. 378.)

Camiguin Mindanao

waldeni Sharpe. (p. 380.)

Guimaras Panay Nẹros

Suborder MEROPES.

Family MEROPIDE:

\section{MEROPS Linn.}

(Sharpe, Cat. Bds., XVII, p. 58, 1892.)

americanus $P$. L. S. Müller, Syst. Nat.

Suppl., p. 95 (1776); teste Sharpe.

[bicolor Bodd. (p. 60.)]

$\begin{array}{ll}\text { Cagayancillo } & \text { Masbate } \\ \text { Calamianes } & \text { Mindanao } \\ \text { Catanduanes } & \text { Mindoro } \\ \text { Cebu } & \text { Negros } \\ \text { Cresta de Gallo } \\ \text { Fuga } & \text { Panay } \\ \text { Guimaras } & \text { Samar } \\ \text { Leyte } & \text { Semirara } \\ \text { Lubang } & \text { Sibuyan } \\ \text { Luzon } & \text { Tablas } \\ \text { Marinduque } & \end{array}$


philippinus Linn. (p. 71.)

\begin{tabular}{ll} 
Malayan Islands \\
Malay Peninsula \\
Indian Peninsula \\
Indo-Chinese provinces \\
Ceylon & \multicolumn{1}{c}{ Celebes } \\
\cline { 2 - 2 } Basllan & Mindoro \\
Bazol & Negros \\
Bohol & Panay \\
Cebu & Samar \\
Guimaras & Sibuyan \\
Leyte & Siquijor \\
Luzon & Sulu \\
Masbate & Tawi Taw \\
Mindanan &
\end{tabular}

\section{Suborder CAPRIMULGI.}

Family CAPRIMULGIDAE.

Subfamily CAPRIMULGIN压.

LYNCORNIS Gould.

(Hartert, Cat. Bds., XVI, p. 603 ; irl.

'Tierr., p. 24.)

macrotis (Tig.). (p. 605.)

[mindanensis Tweed. (1. c.)]

Basilan Mindanao

Luzon Mindoro

\section{CAPRIMULGUS Linn.}

(Hartert, Cat. Bds., XVI, p. 521 ; id. Tierr., p. 3\%.)

griseatus Wald. (p.550.)

Catanduanes Negros

Luzon Sibuyan

Mindoro

mindanensis (Mearns), Proc. Biol. Soc. Mindanao.

Wash., XVIJI, p. 85 (1905).

manillensis Wald. (p. 544.)

$\begin{array}{ll}\text { Basllan } & \text { Negros } \\ \text { Luzon } & \text { Palawan } \\ \text { Masbate } & \text { Romblon } \\ \text { Mindanao } & \text { Ticao } \\ \text { Mindoro } & \end{array}$

macrurus Horsf. (p. 537.)

Papuan Islands

Northeastern Australia

Greater Sunda Islands (Java)

Calamianes Palawan

jotaka Tcmm. of Schl. (p. 5.52.)

Northeastern Siberia

Chlna Japan

New Guinea (winter)

Indo-Chinese provinces

Malay Peninsula (winter)

Greater Sunda Islands (winter)

Calayan Palawan 


\section{Suborder CYPSELI.}

Family MACROPTERYGIDE.

MACROPTERYX Swains.

(Hartert, Cat. Bds., XVI, p. 512, 1892 ; id. Tierreich, Lief. I, p. 63 , 189\%.)

major Hartert, Nov. Zool., IV, p. 11, ex Dubois.

[comata Temm.; Cat. Bds., XVI, p. 512, pt.]

$\begin{array}{ll}\text { Basilan } & \text { Negros } \\ \text { Cebu } & \text { Panay } \\ \text { Guimaras } & \text { Samar } \\ \text { Luzon } & \text { Sibutu } \\ \text { Marinduque } & \text { Sulu } \\ \text { Masbate } & \text { Tablas } \\ \text { Mindanao } & \text { Tawi Tawi } \\ \text { Mindoro } & \end{array}$

Family CYPSELIDFE.

Subfamily CHAETURIN平.

SALANGANA Geoffr. St.-Hilaire.

(Collocalia Gray; Hartert, Cat Bds., XVI, p. 498, 1892; id. Tierr., p. 66.)

whiteheadi Grant, Ibis, 1895, p. 459.

(Tierr., p. 6.)

Cagayancillo Sibuyan

Luzon Palawan

Mindanao Verde

Mindoro

lowi (Sharpe). (p. 498.)

Northern Borneo

Nias Island Sumatra

Palawan

fuciphaga (Thunb.). (p. 498.)

Seychelles Islands

Islands of western Polynesia

Indo-Malayan and Papuan Islands

$\begin{array}{ll}\text { Cebu } & \text { Negros } \\ \text { Luzon } & \text { Palawan } \\ \text { Mindanao } & \text { Panay } \\ \text { Mindoro } & \end{array}$

francica $(G m$.$) . \quad (р. 503.)$

Mauritius

Solomon Islands

Molucea Islands

Reunion Island

Northern Australia

Southern New Guinea

Islands of western Polynesia

Cagayancillo Cuyo

Cagayan Sulu Negros

Calamianes Panay 
inexpectata Hume. (p. 505.)

troglodytes Gray. (p. 507.)

linchi Horsf. \& Moore. (p. 508.)

marginata Salvad. (p. 508.)

[cebuensis Kutter, teste Dubois, Syn. Av., p. 130.]

CHETURA Steph.

(Hartert, Cat. Bds., XVI, p. 470, 1892 ; id. Tierr., p. $70,189 \%$ )

gigantea (Temm.). (p. 475.)

celebensis Scl. (p. 476.)

dubia McGregor, Govt. Lab. Publ. No. 34, p. 15, pl. xii (1905).

picina Tweedd. (p. 487.)

\author{
Andaman Islands \\ Southern Tenasserim \\ Western Malay Peninsula \\ Cagayan Sulu
}

$\begin{array}{ll}\text { Cebu } & \text { Negros } \\ \text { Guimaras } & \text { Palawan } \\ \text { Leyte } & \text { Panay } \\ \text { Luzon } & \text { Romblon } \\ \text { Marinduque } & \text { Samar } \\ \text { Masbate } & \text { Sibuyan } \\ \text { Mindanao } & \text { Siquijor } \\ \text { Mindoro } & \end{array}$

Sumatra

Nicobar Islands

Andaman Islands

Malay Peninsula

Java

Borneo

Bongao Mindanao

Luzon Mindoro

Calayan Masbate

Cebu Mindoro

Luzon Sibuyan
Sumatra

Malay Peninsula

Java

Borneo

Calamianes

Palawan

Celebes

Basilan

Negros

Mindoro

Leyte

Mindanao

Subfamily CYPSELIN㤅.

TACHORNIS Gosse.

(Hartert, Cat. Bds., XVI, p. 462, 1892.)

pallidior McGregor, Govt. Lab. Publ. No. 25, - Luzon

p. 27 (1904). 


\section{Order TROGONES.}

\section{Family TROGONIDE.}

PYROTROGON Bp., Consp. Av., Zygod. p. 14

(1854).

[Harpactes Swains. (nec. Templeton); Grant, Cat. Buls., XVIT, p. 480, 1892. Cf. Oberholser, Proc. Philacl. Acat., 1899, p. 206. 1

ardens $(T \mathrm{cmm}$.$) . (p. 4ST.)$

$\begin{array}{ll}\text { Basilan } & \text { Marinduque } \\ \text { Dinagat } & \text { Mindanao } \\ \text { Leyte } & \text { Samar }\end{array}$

Suborder CUCULI.

Family CUCULIDA.

Subfamily CUCULIN 2 .

COCCYSTES Gloger.

(Shelley, Cat. Bils., XIX-, p. 211, 1891.)

coromandus (Linn.). (p. 214.)

Malay Peninsula

Burmese countries

Sumatra Borneo

Java Celebes

Indian Peninsula and Ceylon

Southern and central China

Mlndanao Siquijor

Palawan

SURNICULUS Less.

(Shelley, Cat. Bds., XIX, p. 226, 1891.)

lugubris (Horsf.). (p. 227.)

South China

Malay Penlnsula

Indian Peninsula

Burmese provinces

Java

Borneo

Ceylon

Sumatra

Balabac

Palawan

velutinus Sharpe. (p. 230.)

$\begin{array}{ll}\text { Basilan } & \text { Negros } \\ \text { Luzon } & \text { Samar } \\ \text { Malamaul } & \text { Sulu } \\ \text { Mindanao } & \text { Tawi Tawi } \\ \text { Mindoro } & \end{array}$


HIEROCOCCYX S. Müll.

(Shelley, Cat. Bds., XIX, p. 231, 1891.)

sparveroides (Tig.). (p. 232.)

fugax (Horsf.). (p. 236.)

CUCULUS Linn.

Borneo

Eastern Siberia

Malay Peninsula

Indian Peninsula

Burmese provinces

China

Calamianes Negros

Luzon Palawan

Greater Sunda Islands (Sumatra, Java, Borneo)

$\begin{array}{ll}\text { Basilan } & \text { Mindanao } \\ \text { Cagayancillo } & \text { Mindoro } \\ \text { Cebu } & \text { Sulu } \\ \text { Luzon } & \end{array}$

(Shelley, Cat. Bds., XIX, p. 2 40 , 1891.)

micropterus Gould. (p. 241.)

Sumatra
Malay Peninsula
Indian Peninsula
Andaman Islands
Burmese provinces
Ceylon $\quad$ Java
China
Japan

Negros

canorus Linn. (p. 245.)

Afrlca (winter)

Europe and northern Asla

Indian Peninsula (winter)

Malayan subregion to Australia (winter)

Calayan Siquijor

Palawan

Eastern Siberia

Indian Peninsula

Andaman Islands

Malay Archlpelago

Burmese provinces

Northeastcrn Australla

Japan China

New Guinea New Britaln

Mindanao Palawan

\section{PENTHOCERYX Cab.}

(Cuculus, pt., Shelley, Cat. Bils., XIX, p. 262, 1891.)

sonnerati Lath. (p. 262.)

Malay Peuinsula

Indian Peninsula

Burmese provinces

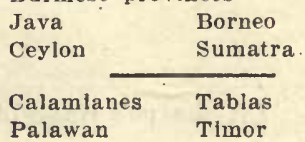


CACOMANTIS S. Müll.

(Shelley, Cat. Bds., XIX, p. 265, 1891.)

merulinus (Scop.). (p. 268.)

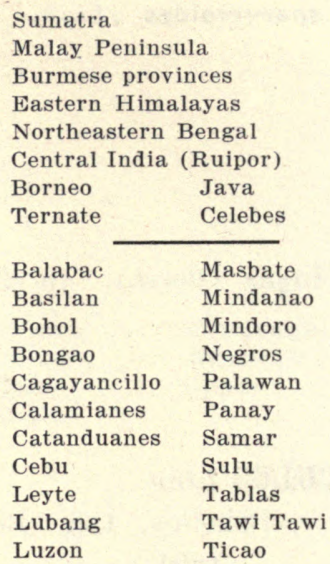

CHALCOCOCCYX $C a b$.

(Shelley, Cat. Bds., XIX, p. 288, 1891.)

xanthorhynchus (Horsf.). (p. 289.)

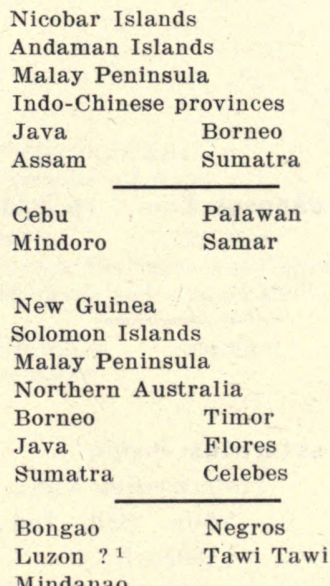

EUDYNAMIS Vig. \& Horsf.

(Shelley, Cat. Bds., XIX, p. 315, 1891.)

honorata (Linn.). (p. 315.)

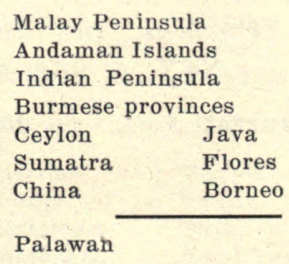

Palawan

${ }^{1} \mathrm{Cf}$. Sharpe's Hand-List of Birds, Vol. II, p. 162, 1900.-WorCESTER. 
mindanensis (Linn.). (p. 321.)

frater McGregor, Bull. Philippine Mus., No.

4, p. 21 (1904).
Sangi Islands

$\begin{array}{ll}\text { Basilan } & \text { Mindoro } \\ \text { Bongao } & \text { Negros } \\ \text { Cagayancillo } & \text { Palawan } \\ \text { Calayan } & \text { Panay } \\ \text { Cebu } & \text { Romblon } \\ \text { Cuyo } & \text { Samar } \\ \text { Fuga } & \text { Semirara } \\ \text { Guimaras } & \text { Sibutu } \\ \text { Leyte } & \text { Sibuyan } \\ \text { Luzon } & \text { Siquijor } \\ \text { Malanipa } & \text { Sulu } \\ \text { Marinduque } & \text { Tablas } \\ \text { Masbate } & \text { Tawi Tawi } \\ \text { Mindanao } & \text { Ticao } \\ \text { Calayan } & \text { Fuga }\end{array}$

Subfamily CENTROPODIN死.

CENTROPUS Illiger.

(Shelley, Cat. Bds., XIX, p. 331, 1891.)

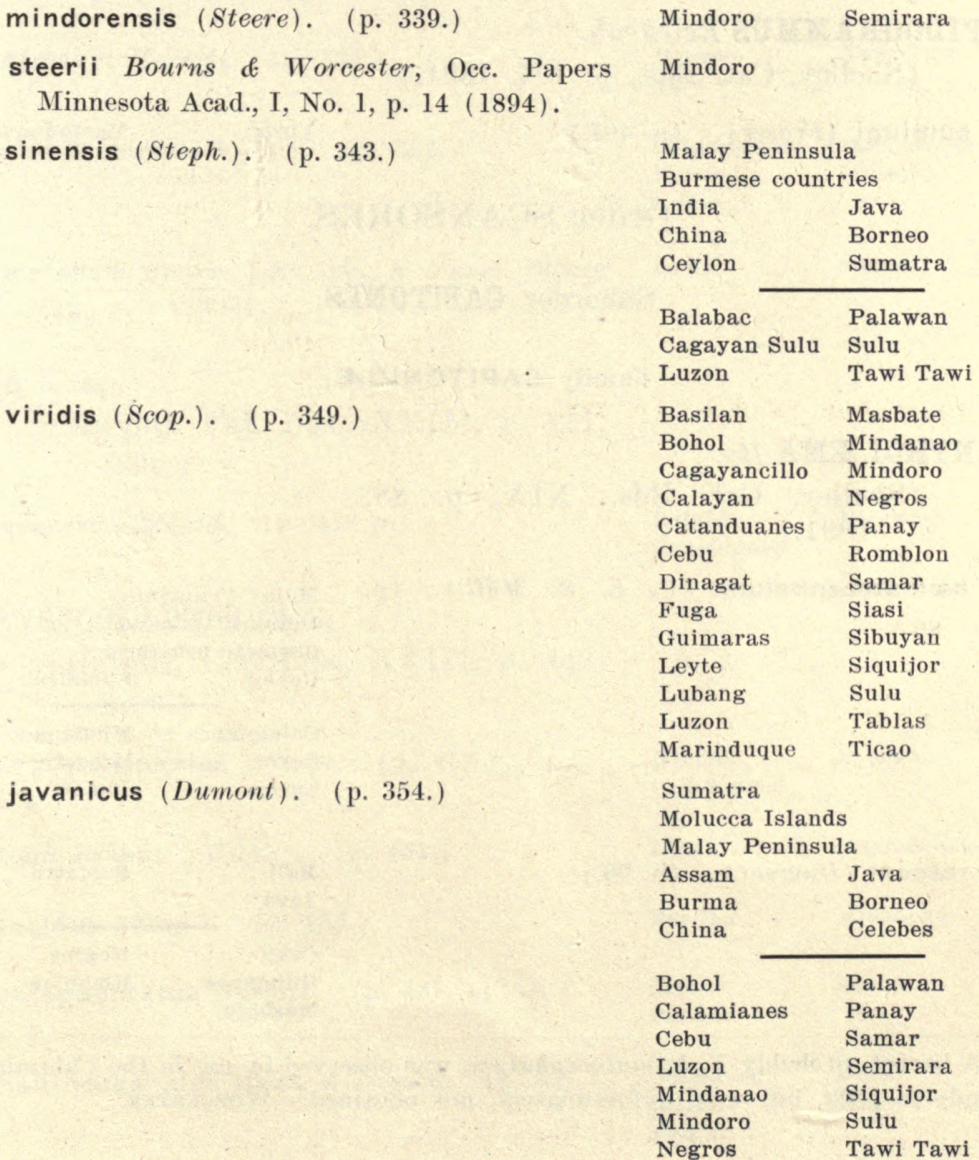


melanops Less. (p. 365.)
Basilan

Leyte

Mindanao

unirufus (Cab. \& Heine). (p. 367.)

Luzon

\section{Subfamily PHENICOPHAIN无.}

DRYOCOCCYX Sharpe.

(Shelley, Cat. Bds., XIX, p. 400, 1891.)

harringtoni Sharpe. (p. 400.)

Balabac

Palawan

Calamianes

DASYLOPHUS Swains.

(Shelley, Cat. Bds., p. 403, 1891.)

superciliosus (Cuv.). (p. 403.)

Catanduanes Marinduque Luzon 


\title{
Order PICIFORMES.
}

\author{
Suborder PICI. \\ Family PICIDAE. \\ Subfunily PICIN $\mathbb{E}$
}

\section{YUNGIPICUS $B p$.}

(Hargitt, Cat. Bds., XVIII, p. 309, 1890.)

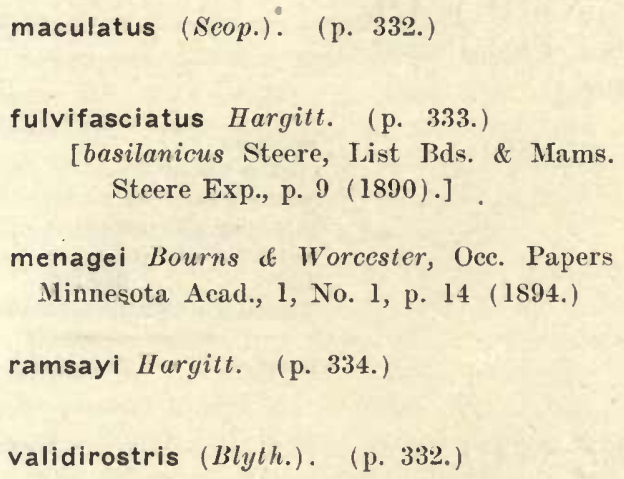

TIGA Kaup.

(Hargitt, Cat. Bds., XVIII, p. 411, 1890.)

everetti Tweedd. (p. 418.)

Balabac

Calamianés

Negros

Guimaras Panay

Basilan

Mindanao

Sibuyan

Bongao

Sulu

Catanduanes

Lubang

Marinduque

Luzon

Mindoro

Leyte

Samar

Palawan

\section{CHRYSOCOLAPTES Blyth.}

(Hargitt, Cat. Bds., XVIII, p. 442, 1890.)

\begin{tabular}{|c|c|c|}
\hline erythrocephalus Sharpe. (p. 452.) & $\begin{array}{l}\text { Balabac } \\
\text { Calamianes }\end{array}$ & Palawan \\
\hline hæmatribon (Wagl.). (p. 454.) & Luzon & Marinduque \\
\hline lucidus (Scop.). (p. 455.) & Basilan & Mindanao \\
\hline rufopunctatus Hargitt. '(p. 457, pl. xii.) & $\begin{array}{l}\text { Leyte } \\
\text { Panaon }\end{array}$ & Samar \\
\hline xanthocephalus Wald. \& Layard. (p. 457.) & $\begin{array}{l}\text { Guimaras } \\
\text { Masbate } \\
\text { Negros }\end{array}$ & $\begin{array}{l}\text { Panay } \\
\text { Tícao }\end{array}$ \\
\hline
\end{tabular}


MICROSTICTUS Ilargitt.

(Hargitt, Cat. Bds., p. 489, 1890.)

fuliginosus (Tweedd.). (1. 492.)

Leyte

Saniar

Mindanao

funebris (Valenc.). (1. 492.)

Catanduanes Marinduque
Luzon

MULLERIPICUS $B p .{ }^{1}$

[Hemilophens Swains. (nec Serville), Hargitt, Cat. Bds., XVIII, p. 494, 1890, Cf. Oberholser, Proc. Philad.

Acad., 1899, p. 204.]

pulverulentus (Temm.). (p. 494.)

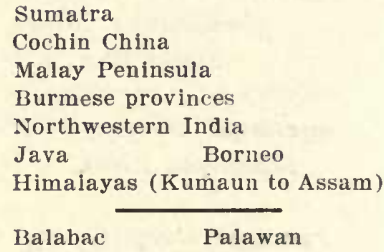

THRIPONAX ${ }^{2}$ Cab. \& Heine, XVIII.

(Hargitt, Cat. Bds., p. 49\%, 1890.)

javensis (Horsf.). (p. 498.)

Malay Peninsula

[suluensis W. Blasius, J. f. O. 1890, p. 140.]

Southern Tenasserin

Sumatra Java

Banka

Borneo

$\begin{array}{ll}\text { Basilan } & \text { Mindanao } \\ \text { Bongao } & \text { Suiu } \\ \text { Cebu } & \text { Surigao } \\ \text { Lapac } & \text { Tawi Tawi } \\ \text { Luzon } & \end{array}$

pectoralis 'T'toeedd. (p. 500, pl. xviii.)

Leyte Samar

Panaon

${ }^{1}$ Sliarpe adopts Alophonerpes Reichenb., rejecting the earlier name Mulleripieus because of its bad formation.-MCGREGOR.

${ }^{2}$ Much uncertainty exists as to the distribntion of the Philippine species of the genus Thriponax. Sharpe, in his Hand-List of Birds, includes T'. javonsis (Horsf.) from Juzon, Negros, Mindanao, and Basilan; T. suluensis Wr. Blasius from Sulu; T. peetoralis Tweedd. from Leyte and Panaon; T. philippensis Stecre from Panay, Guimaras, and Masbate; T'. hargitti sharpe from Palawan, and T. mindorensis Steere from Mindoro. This arrangement leaves entirely out of account the Samar bird, which is undoubtedly typical $T$. peetoralis, as woll as the Bongao, Tawi Tawi, and Cebu representatives of the genus.

Sharpe has himself identified three specimens from Bongao and Tawi Tawi as T. javensis (Ibis, Apr., 1894, p. 249), and the specimens collected in Tawi Tawi by Bourns and myself were by us referred to this species [Occ. Papers Minnesota Acad., I, No. 1, p. 36 (1894) ]. 
$\begin{array}{cll}\text { hargitti Sharpe. (p. 505.) } & \text { Guimaras } & \text { Panay } \\ \text { [philippensis Steere, List 13ds. \& Mams. } & \text { Masbate } & \text { Palawan } \\ \text { Steere Exp., p. } 8(1890) .] & \end{array}$

mindorensis Stcere, List Bis. \& Manss. Miudoro

Steere Exp., p. 8 (1890).

The name T'. suhensis has been given to the Sulu representatives of the genus by W. Blasius (J. f. O. 1890, p. 140), who bases his determination on five specinens collected by Platen. He separates $T$. suluensis from $T$. javensis on the ground that the bills and wings of the Sulu birds are shorter than are those of typical representatives of $T$. javensis. If the latter species, which ranges through Borneo, Bongao, Tawi Tawi, and Lapac, really gives way in Sulu to a distinct species only to reappear again in Basilan and Mindanao, it would be remarkable, although perhaps not more remarkable than that $T$. javensis of Mindanao should be replaced by $T$. peetoralis in Panaon, Leyte, and Samar, and shonld reappear in Lnzon. In point of fact, Bourns and I were quite unable to differentiate our Sulu specimens from typical $T$. juvensis, and 1 must therefore decline to recognize $T$. suluensis of Blasius as a valid species.

The question presented by the central Philippine representatives of the genug can not be so readily disposed of. Dr. Steere separated the birds from Guimaras and Masbate under the name of $T$. philippensis, differentiating them from T. javensis on the strength of their possessing bnffy white rumps and cheeks largely searlet [list Bds. \& Mams. Steere Exp., p. 8 (1890)]. Bourns and I assigned our Panay specimens to this species, which we lescrihed somewhat more fully than did Steere [Occ. Papers Minnesota Acad., I, No. 1, p. 53 (1894)].

Later, however, in preparing our distribution list, having noted that Hargitt, in Volume XVIII of the Catalogue of Birds, did not recognize $T$. philippensis, we discarded the species and recorded all the central Philippine representatives of the genus, including our Negros specimens, under $T$. javensis.

William Eagle Clark unhesitatingly identified an adult male bird collected in Negros by Keay as $T$. hargitti (Ibis, Oct., 1894, p. 534). In lbis, Oct., 1895, p. 474, Clarke quotes Hargitt's views as to the identity of this specimen. Hargitt failed to reach any definite conclusion, but suggested that it resembled $T$. phitippensis more closely than $T$. hargitti, and added that he regarded the only other specimen that he had seen from Negros as T. javensis.

Grant (Ibis, Oct., 1894, p. 473) asserts that T. philippensis Stecre is identical with $T$. hargitti Sharpe, and later reiterates this statement (Ibis, Oct., 1896, p. 558), but adds to our confusion by saying that the bird from Negros recorded by Hargitt under the name of $T$. javensis (Cat. Bds., XVIII, 1. 500, specimen X) should, in his opinion, be referred to $T$. pectoralis, all the feathers of its breast being widely margined with whitislı buff. Finally Clark (Ibis, Jan., 1898, p. 121) identifies three additional specimens from Negros as T'. hargitti.

As above noted, Sharpe, in his Hand-List of Birds, refers the Negros birds to $T$. javensis and retains Steere's T. philippensis, to which species he refers the birds from Panay, Guimaras, and Masbate.

The question of the identity of the central Philippine representatives of the genus can hardly be satisfactorily settled without comparing a good series of specimens with a similar series from Palawan. Meanwhile, in view of Grant's positive statement that the types of $T$. hargitti and $T$. philippensis have been compared and agree in all particulars, I refer the central Philippine representatives of the genus to this species.-WORCEsTER. 


\title{
Order EURYLAMIFORMES.
}

\section{Family EURYLAEMIDAE.}

Subfamily EURYLEMIN瓜.

\section{SARCOPHANOPS Sharpe.}

\author{
(Selater, Cat. Bds., XIV, p. 462, \\ 1888.) \\ steerei Sharpe. (p. 462.) \\ Basilan \\ Mindanao \\ samarensis Steere, List Bds. \& Mams. Steere \\ Leyte \\ Samar
}

Exp., p. 23 (1890).

\section{Order PASSERIFORMES.}

\section{Suborder MESOMYODI.}

\section{Family PITTIDAE.}

PITTA Vieill.

(Sclater, Cat. Bds., XIV, p. 413, 1888.)

erythrogaster Temm. (p. 432.)

propinqua Sharpe. (p. 433.)

Basilan Palawan

Bongao Panay

Cagayancillo Romblon

Calamianes Samar

Cebu Sibutu

Guimaras Sibuyan

Lubang Siquijor

Luzon Sulu

Marinduque Tablas

Masbate Tawi Tawi

Mindanao Ticao

Mindoro

Palawan

kochi Brüggem. (p. 433.)

Luzon

atricapilla Less. (p. 438.)

$\begin{array}{ll}\text { Balabac } & \text { Palawan } \\ \text { Basilan } & \text { Romblon } \\ \text { Bongao } & \text { Samar } \\ \text { Calamianes } & \text { Semirara } \\ \text { Cebu } & \text { Sibuyan } \\ \text { Luzon } & \text { Siquijor } \\ \text { Marinduque } & \text { Sulu } \\ \text { Mindanao } & \text { Tablas } \\ \text { Mindoro } & \text { Tawi Tawi } \\ \text { Negros } & \end{array}$

mülleri $B p . \quad$ (p. 439.)

$\begin{array}{ll}\begin{array}{l}\text { Banka } \\ \text { Borneo }\end{array} & \text { Sumatra } \\ \text { Sibutu } & \\ \text { Samar } & \text { Mindanao }\end{array}$




\section{Suborder ACROMYODI.}

Family HIRUNDINIDE.

Subfamily HIRUNDININ Æ.

\section{CHELIDONARIA Reichenow.}

(Reichenow, J. f. O. 1889, p. 18\%.)

(Chelidon Boie (nec Forst.);

Sharpe, Cat. Bds., X, p. 85, 1885.)

dasypus (Bp.). (p. 91.)

Borneo (winter)

Calayan

CLIVICOLA Forster.

(Cotile Boie; Sharpe, Cat. Bds., X, p. 96,1885 .)

riparia (Linn.). (p. 96.)

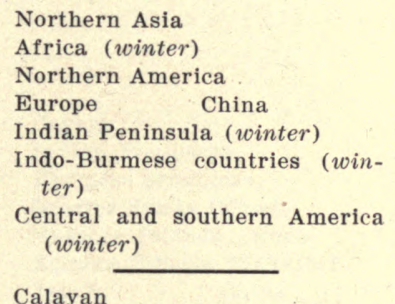

sinensis (J. E. Gray). (p. 104.)

\author{
Formosa \\ Southern China \\ Indian Peninsula (winter) \\ Indo-Chinese countries (win- \\ ter)
}

Luzon (winter)

\section{HIRUNDO Schaeffer (ex Linn.)}

(Sharpe, Cat. Bds., X, p. 123, 1885 ;

Sharpe, \& Wyatt, Monogr. Hirund., I, p. 209.)

rustica Linn. (p. 128.) 
gutturalis Scop. (p. 134.)

javanica Sparrm. (p. 142.)

striolata Boie. (p. 161.)
Malay Peninsula

Malay Archipelago

Indo-Chinese provinces

Southern China (winter)

Central and eastern Siberia

Northern Australia (winter)

Kamtchatka China

Japan

New Guinea

Basilan Mindanao

Cagayan Sulu Mindoro

Calayan Palawan

Cuyo Panay

Fuga Semirara

Guimaras Sibay

Luzon Siquijor

Maestro de Sulu

Campo Ticao

New Guinea

Southern India

Molueca Islands

Malay Peninsula

Islands of Torres Straits

Java Borneo

Ceylon Sumatra

Basilan Mindanao

Bohol Mindoro

Cagayan Sulu Negros

Calamianes Palawan

Catanduanes Panay

Cebu Romblon

Cuyo Samar

Dinagat Sibutu

Guimaras Sibuyan

Leyte Siquijor

Lubang Sulu

Luzon Tawi Tawi

Marinduque Ticao

Masbate

\section{Formosa}

Southern China

Burmese provinces

Java Flores

Assam Cachar

Calayan Masbate

Luzon

Family MUSCICAPIDAE.

HEMICHELIDON Hodgs.

(Sharpe, Cat. Bds., IV, p. 111, 1879.)

sibirica $(G m$.$) . (p. 120.)$

\author{
Japan \\ China (winter) \\ Eastern Siberia \\ Altai Mountains \\ Palawan
}


griseisticta (Swinh.). (p. 153.)

ferruginea Hodgs. (p. 132.)

[cinereiceps Sharpe, Ibis, 1887, p. 141; Cf. Sharpe, Ibis, 1894, p. 541.]

\section{ALSEONAX $C a b$.}

(Sharpe, Cat. Bds., IV, p. 126, 18\%9.)

latirostris (Raffles). (p. 127.)

Ceylon
Indian Peninsula
Burmese provinces
Greater Sunda Islands
Molucca Islands (Buru)
Eastern Siberia (Sakalin)
Japan China
Bongao ? ${ }^{1}$ Sulu ?1
Negros

\section{CYORNIS Blyth.}

(Siphia, pt., Sharpe, Cat. Bds., IV, p. 441, 18\%9). [Cf. Oates, Faun. Brit. Ind., Birds, II, p. 11 (1890).]

herioti Wardlaw-Ramsay, Ibis, 1886, p. 159 Luzon

(우).

[enganensis Grant, Bull. B. O. C., IV, p. ii (1895). Cf. Sharpe, Bull. B. O. C., XI, p. 60 (1901.)]

banyumas (Horsf.). (p. 449.)

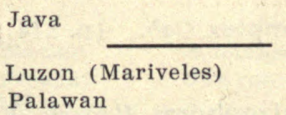

${ }^{1}$ Sharpe records A. latirostris from Bongao and Sulu [Hand-List of Birds, Vol. III, p. 206 (1901)]. In his list of the birds of the Sulu Archipelago published in Tbis, April, 1894, pp. 257-259, he does not include this species. 
philippinensis Sharpe. (p. 450.)
[= simplex Blytl, Ibis, 1870, p. 165 ,
teste Finsch in litt.].

lemprieri Sharpe, Ibis, 1884, p. 319.

[ramsayi W. Blasius, Ornis, 1888, p. 308. Cf. Everctt, Ibis, 1895, p. 25.]

erythaca Sharpe, Ibis, 1888, p. 199.

[platence Blasius, Ornis, 1888, p. 308.]

MUSCICAPULA Blyth.

(Sharpe, Cat. Bds., IV, p. 203, 1879.)

[Dendrobiastes Sharpe, Tr. Linn. Soc., (2) I, p. 332 (1876).]

samarensis Bourns.\& Worcester, Occ. Papers Minnesota Acad., I, p. 24 (1894).

Iuzoniensis Grant, Ibis, 1894, p. 505.

nigrorum Whitchead, Bull. B. O. C. vi, p. xliii (1897).

- montigena Mcarns, Proc. Biol. Soc. Wash., XV11I, p. 8 (1905).

basilanica Sharpe, Tr. Linn. Soc., $2 d$ ser, i, p. 332 (1876).

[mindanensis W. Blasius, J. f. O. 1890, p. 147. Cf. Sharpe, Bull. B. O. C. xi, p. 60 (1901).]

westermanni Sharpe, P. Z. S., 1888, p. 270.

\section{GERYGONE Gould.}

(Sharpe, Cat. Bds., IV, p. 211, 1879.)

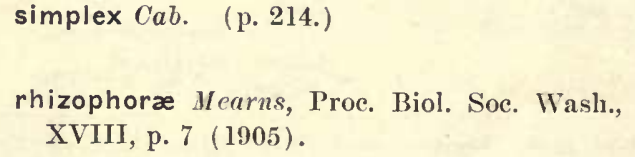

$\begin{array}{ll}\text { Basllan } & \text { Panay } \\ \text { Bongao } & \text { Romblon } \\ \text { Cebu } & \text { Samar } \\ \text { Dinagat } & \text { Semirara } \\ \text { Guimaras } & \text { Sibutu } \\ \text { Leyte } & \text { Sibuyan } \\ \text { Luzon } & \text { Siquijor } \\ \text { Marinduque } & \text { Sulu } \\ \text { Masbate } & \text { Tabias } \\ \text { MIndanao } & \text { Tawi Tawi } \\ \text { Mindoro } & \text { Ticao } \\ \text { Negros } & \end{array}$

Balabac Palawan

Caiamianes

Palawan

Samar

$\begin{array}{ll}\text { Calayan } & \text { Mindoro } \\ \text { Luzon } & \text { Negros ? }\end{array}$

Negros

MIndanao

Basilan

Mindanao

Malay Peninsula (Mountains of Perak)

Mountains of northwestern Borneo and Celebes

Luzon Negros
Mindanao

$\begin{array}{ll}\begin{array}{l}\text { Lubang } \\ \text { Luzon }\end{array} & \begin{array}{l}\text { Mindoro } \\ \text { Verde }\end{array} \\ \text { Bongao ? } & \text { Sulu ? } \\ \text { Mindanao } & \end{array}$

${ }^{i}$ Sulu and Bongao birds probably belong to the race rhizophorc. Guillemard refers his Sulu birds to flaveola. Cf. P. Z. S., 1885, p. 263.-McGregor. 
XANTHOPYGIA Blyth.

(Sharpe, Cat. Bds., IV, p. 249, 1879.)

narcissina (Temm.). (p. 249.)

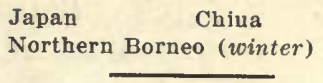

Mindanao Mindoro

CYANOPTILA Blyth.

(Xanthopygia pt., Sharpe, Cat. Bds., IV, p. 249, 18г9.)

bella (A. Hay). (p. 251.)

Northwestern Borneo

[Muscicapa cyanomelana Temm., nee M. cyanomclas Vieill.]

[Cf. Stejn., Pr. U. S. Nat. Mus., xv, p. $328(1892)$.

Japan Cbina

Indo-Chinese countries

Palawan (vinter)

\section{HYPOTHYMIS Boie.}

(Sharpe, Cat. Bds., IV, p. 273, 1879.)

occipitalis (Vig.). (p. 275.)

\begin{tabular}{ll} 
Nicobar Islands \\
Malay Penlnsula \\
Java & \multicolumn{1}{l}{ Flores } \\
Formosa & Hainan \\
Borneo & Lombok \\
\cline { 2 - 2 } Basilan & Mindoro \\
Bohol & Negros \\
Bongao & Palawan \\
Calamianes & Panay \\
Cebu & Romblon \\
Guimaras & Samar \\
Leyte & Sibutu \\
Lubang & Sibuyan \\
Luzon & Siquijor \\
Ma estro de & Sulu \\
Campo & Tablas \\
Malanipa & Tawi Tawi \\
Marinduque & Ticao \\
Masbate & Verde \\
Mindanao &
\end{tabular}

superciliaris Sharpe. (p. 278.)

Basilan Mindanao

samarensis Steere, List Bds. \& Mams. Philip- Leyte

Samar pine Exp., p. $16 \cdot(1890)$.

\section{CYANOMYIAS Sharpe.}

(Sharpe, Cat. Bds., IV, p. 273, 1879.)

cœlestis (Tuectl.). (p. 278.)

Basilan Mindanao

Dinagat Sibuyan

Luzon

Samar

helenæ Stcore, List Bds. \& Mams. Philippine Exp., p. 10 (1890). 
RHIPIDURA Vig. \& Horsf.

(Sharpe, Cat. Bds., IV, p. 303, 1879.) $[C f$. Büttik. Notes Leyden Mus., xv, p. 65 (1893).]

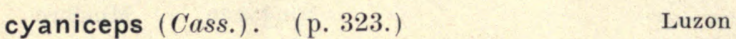

$\begin{array}{ll}\text { Basilan } & \text { Negros } \\ \text { Bohol } & \text { Palawan } \\ \text { Bongao } & \text { Panay } \\ \text { Calamianes } & \text { Romblon } \\ \text { Cebu } & \text { Samar } \\ \text { Cuyo } & \text { Siasi } \\ \text { Dinagat } & \text { Sibuyan } \\ \text { Guimaras } & \text { Siquijor } \\ \text { Lubang } & \text { Sulu } \\ \text { Luzon } & \text { Tablas } \\ \text { Marinduque } & \text { Tawi Tawi } \\ \text { Masbate } & \text { Ticao } \\ \text { Mindanao } & \text { Verde } \\ \text { Mindoro } & \end{array}$

\section{ZEOCEPHUS $B p$.}

(Sharpe, Cat. Bds., IV, p. 342, 18\%9.)

rufus (Gray). (p. 343.)

$\begin{array}{ll}\text { Cebu } & \text { Panay } \\ \text { Lubang } & \text { Romblon } \\ \text { Luzon } & \text { Samar } \\ \text { Marinduque } & \text { Sibuyan } \\ \text { Mindoro } & \text { Tablas } \\ \text { Negros } & \end{array}$

cinnamomeus Sharpe. (p. 343.)

$\begin{array}{ll}\text { Basilan } & \text { Sulu } \\ \text { Bongao } & \text { Tawi Tawi }\end{array}$

cyanescens Sharpe. (p. 343.)

\section{CALLAEOPS Grant.}

(Grant, Bull. B. O. C., IV, pp. xviii, xxii, 1895.)

periophthalmica Grant. (1. c.) 
TERPSIPHONE Gloger.

(Sharpe, Cat. Bds., IV, p. 344, 18\%9.)

affinis (Blyth.). (p. 349.)

Malay Peninsula

Eastern Himalayas

Indo-Chinese provinces

Assam Java

Borneo

Sumatra

Luzon

\section{RHINOMYIAS Sharpe.}

(Sharpe, Cat. Bds.. IV, p. 367, 18\%9.)

albigularis Bourns of Worcester, Oce. Papel's Mimnesota Acad., I, No. 1, p. 27 (1894).

ruficauda (Sharpe). (p. 368.)

[samarensis Steere, List. Bds. \& Mams. Stecre Exp., p. $16(1890)$.

ocularis Bourns of Woreester, Oce. Papers

Minnesota Aead., I, No. 1, p. 28 (1894).

insignis Grant, Bull. B. O. C., IV, p. xl (1895) ; id. Ibis, 1895, p. 442, pl. xii, fig. 2.

CULICICAPA Swinh.

(Sharpe, Cat. Bds., IV, p. 369, 18\%9.)

ceylonensis (Swains.). (p. 369.)

helianthea (Wall.). (p. 370.)

[panayensis Sharpe. (p. 371.)]
Guimaras Negros

Basilan Mindanao

Leyte Samar

Sulu

Tawl Tawi
Luzon
Khasl Hills

Indian Peninsula

Mountains of northwesteru Borneo

Malay Peninsula (Mountains of Perak)

Ceylon

Java

Palawan

Celebean Sea Celebes

Banggal and Saleyer Islands

Bongao Negros

Cebu Palawan

Leyte Panay

Luzon Tawl Tawl

Mindanao

CRYPTOLOPHA Swains.

(Sharpe, Cat. Bds., IV, p. 393, 18\%9.) [Cf. Oates, Faun. Brit. Ind., Birds, I, p. 421, 1889.]

cebuensis Dubois, Syn. Av. p. 286 (1900). Cebu

Ifavigularis Bourns \& Worcester, Oce. Papers Minnesota Acad., I, No. 1, p. 23 (1894, nee Godw.-Aust.).] 
montis Sharpe, Ibis, 1887, p. 442.

xanthopygia Whitehead, Bull. B. O. C., I, p. xxxi (1893) ; id. Exped. Kina Balu, pl. 16, fig. 2 (1893).

mindanensis Hartert, Bull. B. O. C., XIV, p. $12(1903)$.

ABRORNIS Hodgs.

(Cryptolopha, pt., Sharpe, Cat. Bds., IV, p. 393, 1879.)

nigrorum (Moseley), Ibis, 1891, p. 47, pl. ii, fig. 1 .

olivacea Moseley, lbis, 1891, p. 4i, pl. ii, fig. 2 .

EUMYIAS $C a b .^{1}$

(Stoparola Blyth; Sharpe, Cat. Bds., IV, p. 435, 18\%9.)

panayensis (Sharpe). (p. 440.)

nigrimentalis (Grant), Bull. B. O. C., III, p. 50 (1894); id., Ibis, 1894, p. 507, pl. xiv, fig. 2 .

nigriloris (Hartert), Bull. B. O. C., XIV, Mindanao p. 80 (1904).
Sumatra

Mountalns of northwestern Borneo

Palawan

Palawan

Mindanao

Luzon

Negros

Mindoro

$\begin{array}{ll}\text { Leyte } & \text { Samar } \\ \text { Luzon } & \text { Sulu }\end{array}$

Mindanao . Tawi Tawi

Negros

Luzon Mindoro

\section{Family CAMPOPHAGIDAE.}

ARTAMIDES Hartl.

(Sharpe, Cat. Bds., IV, p. 8, 18\%9.)

dificilis Hartert, Nov. Zool., II, p. 470 (1895).

Balabac

Palawan

Calamianes

guillemardi Salvad., Ibis, 1886, p. 154.

Bongao

[pollens (nec Salvad.) Guillem., P. Z. S.,

Lapac

Sibutu

Sulu 1885, p. 258.]

striatus (Bodd.). (p. 18.)

Lubang

Luzon

kochi Kutter, Orn. Centralbl., 1882, p. 183;

Basllan

Nipa

J. f. O. 1883 , p. 163.

Leyte

Panaon

[mindanensis Steere, List Bds. \& Mams.

Mindanao

Samar 
panayensis stcere, List. Bds. \& Mams. Steere Exp., p. 14 (1890).

Guimaras

Masbate

Panay

Negros

mindorensis Stecre, t. e., p. 14 (1890).

Mindoro

Tablas

cebuensis Grant, Ibis, 1898, p. 535.

Cebu

EDOLIISOMA Jacq. \& Pucher.

(Sharpe, Cat. Bds., IV, p. 42, 18\%9.)

cærulescens (Blyth). (p. 44.)

Luzon

.alterum Wardlaw-Ramsay, Ibis, 1881, p. 34.

Cebu

panayense Steere, List Bds. \& Mams. Steere Exp., p. 14 (1890).

Guimaras

Panay

Negros

everetti Sharpe, Bull. B. O. C., III, p. x Bongao

Tawi Tawi

(1893).

mindanense (Tweedd.) (P. Z. S., 1878, p.

Sulu

Basilan

Mindanao 947 ) ; Sharpe, Cat. Bds., IV, p. 470 ( 1879 ).

elusum MeGregor, Govt. Lab. Publ. No. 34, Mindoro p. $19(1905)$.

\section{PERICROCOTUS Boie.}

(Sharpe, Cat. Bds., IV, p. \%0, 1879.)

marchesæ Guillem., P. Z. S., 1885, p. 259, Sulu pl. xviii, fig. 1 .

novus MeGregor, Bull. Plilippine Mus., No.

Luzou

Negros

3, p. 13 (1904); W. Ramsay, lbis, 1886, p. 161 .

leytensis steere, List Bds. \& Mams. Steere Exp., p. 15 (1890).

Leyte

Samar

igneus Blyth. (p. 78.)

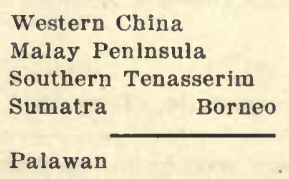

cinereus Lafr. (p. 83.)
China

Pegu (winter)

Eastern Siberia

Borneo (winter)

Malay Peninsula (winter)

Calayan

Luzon

Mindoro Palawan 
LALAGE Boic.

(Sliarpe, Cat. Bds., IV, p. 86, 18r9.)

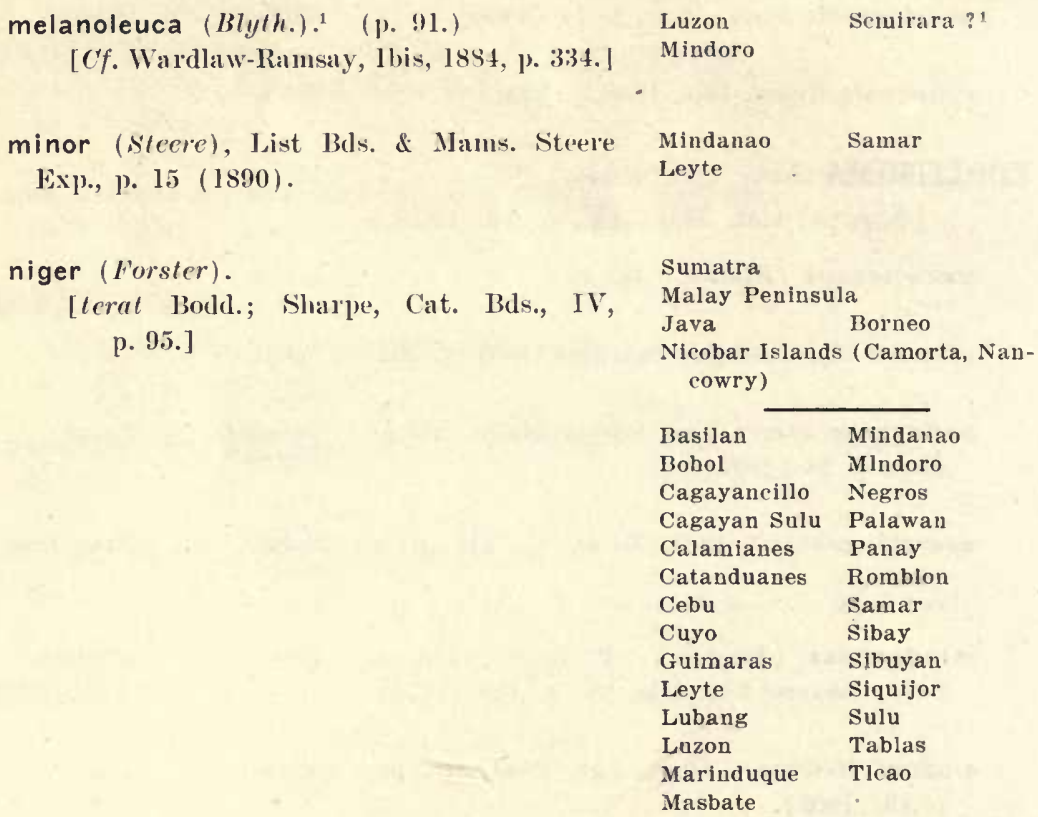

Family PYCNONOTIDAE.

ÆGITHINA Vicill.

(Sliarpe, Cat. Bds., VI, p. 4, 1881.)

viridis $(B p$.$) (p. 11.)$

Sumatra Borneo

Palawan

CHLOROPSIS Jard. d Selby.

(Sharpe, Cat. Bds., VI, p. 15, 1881.)

palawanensis Sharpe. (p. 33.

Baiabac Palawan

Calamianes

Mindanao ?:

flavipennis ('Treedl.) (p. 34 .

Cebu

${ }^{1}$ A bird seen by me on Semirara in April, 1905, but not killed, was in all probability L. melanoleuca.-Worcester.

${ }^{2}$ Chloropsis flavipennis is listed by W. Blasius among the birds collected by Dr. Platen at Davao, in Mindanao, in the summer of 1889 (J. f. O., April, 1890, p. 145). The failure of any other collector to find any representative of this genus in Mindanao is so renarkable that it leads me to doubt the correctness of this record which Sharpe does not recognize in his Hand-List of Birds.Worcester. 
IRENA IIorsf.

(Sharpe, Cat. Bds., VI, p. 184, 1881.)

cyanogaster Vig. (p. 175.)

Luzon

ellæ Steere, Jist Bds. \& Mams. Steere Fxp., Leyte

Samar p. $18(.1890)$.

melanochlamys sharpe. (p. 170.)

Basilan

Mindanao

tweeddalii sharpe. (p. 178.)

Balabac

Palawan

Calamianes

HYPSIPETES Vig.

(Sharpe, Cat. Bds., VI, p. 35, 1881.)

fugensis (irant, Bull B. O. C., V, p. ii (1895); icl. Ibis, 1896, p. 113.

Calayan

Fuga

IOLE Blyth.

(Sharpe, Cat. Bds., VI, p. 5t, 1881.)

striaticeps Sharpe, 1lbis, 1888, p. 200.

Palawan

everetti Tucedd. (p. 57.)

Mindanao

Dinagat

Panaon

Leyte

haynaldi Blasius. J. f. O., 1890, p. 141;

Sharpe, Ibis, 1894, p. 253.

Bongao

Sibutu

Suiv

rufigularis (Sharpe). (p. 57, pl. iii.)

Basilan

Mindanao

philippensis $(G m$.$) . (p. 58.)$

Bohol

Cebu

Leyte

Lubang

Samar

Luzon

guimarasensis steere, Bds. \& Mans. Steere

Guimaras

Masbate

Negros

lixp., p. $19(1890)$.

mindorensis Stecre, List. Bds. \& Miums.

Mindoro

Verde

Steere Exp., p. 19 (1890).

[schmackeri Hartert, J. f. O., 1890, p. 155.1

siquijorensis steere, op. cit. p. 19 (1890).

Siquijor

cinereiceps Bourns a Wrorcester, Oec. Paper's

Kombion

Tablas

Minnesota Aead., 1, No. 1, p. 25 (1894).

monticola Bourns of Worcester, l. c.

Cebu

POLIOLOPHUS Sharpe.

(Sharpe, Tr. Iinn. Soc. (2) i, p. 334, 1876$.

urostictus (Salvad.). (p. 63.)

[basilanicus Steere, List. Bds. \& Mams. Steere Exp., p. 19 (1890).]

Basilan

Catanduanes

Dinagat

Leyte
Luzon Mindanao

Panaon

Samar 
MICROTARSUS Eyton.

(Micropus Swains., nec Limn.; Sharpe, Cat. Bds., VI, p. 64, 1851.)

melanocephalus (Gm.). (p. 66.)

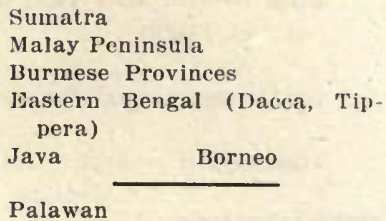

CRINIGER Temm.

(Sharpe, Cat. Bds., VI, p. \%0, 1881.) frater Sharpe. (p. 79, pl. v.)

palawanensis Tweedd. (p. 83, pl. vi, fig. 2.)

\section{PYCNONOTUS Boie.}

(Sharpe, Cat. Bds., VI, p. 120, 1881.)

「Pachycephatixus \& Stictognathus,

Buittik. Notes Leyden Mus., xvii, p. $249(1896)$.

Gymnocrotaphus \& Bonapartea, Biittik., op. cit. xvii, p. 246, xviii, p. 58.]

goiavier (Scop.). (p. 141.)

cinereifrons Twecdd. (p. 153.)
Balabac Palawau

Calamianes

Palawan

$\begin{array}{ll}\text { Basilan } & \text { Negros } \\ \text { Bobol } & \text { Nipa } \\ \text { Cebu } & \text { Panay } \\ \text { Gulmaras } & \text { Romblon } \\ \text { Leyte } & \text { Samar } \\ \text { Luzon } & \text { Sulu } \\ \text { Marinduque } & \text { Tablas } \\ \text { Masbate } & \text { Ticao } \\ \text { Mindanao } & \text { Verde } \\ \text { Mindoro } & \\ \text { Calamianes } & \text { Palawan }\end{array}$

Family TIMELIIDAE.

Subfamily TIMELIIN无.

\section{PSEUDOTHARRHALEUS Grant.}

(Grant, Bull. B. O. C., IV, p. xl, 1895.)

caudatus Grant, $l$. $c$.

Luzon

unicolor Hartert, Bull. B. O. C., XIV, p. 74 MIndanao (1904). 
griseipectus Mcurns, Proc. Biol. Soc. Wash., Mindauao XV111, p. 2 (1905).

TURDINUS Blyth.

(Sharpe, Cat. Bds., VII, p. 539, 1883.)

[Cf. Büttik. Notes Leyden Mus. XVII, p. 68 (1895). Malacocincla Büttik. t. c. p. 79, Illadopsis Büttik, t. c. p. 98$.

rufifrons Tweedd. (p. 546.)

Balabac Palawan

[Malacopterum palawanense Büttik. Notes Leyden Mus. XVII,. p. 104 (1895).]

PTILOCICHLA Sharpe.

(Sharpe, Cat. Bds., VII, p. 586, 1883.)

falcata Sharpe. (p. 586.)

Balabac

Palawan

basilanica Steere, List. Bds. \& Mams. Steere

Basllan Exp., p. 18 (1890); id. Ibis, 1891, pl. vii.

mindanensis Steere, l. c.; $C f$. Blasius, J. f. O. 1890 , p. 146.

minuta Bourns \& Worcester, Occ. Papers Samar Minnesota Acad., I, No. 1, p. 24 (1894).

\section{ANUROPSIS Sharpe.}

(Sharpe, Cat. Bds., VII, p. 5ss, 1883.)

cinereiceps (Tweedd.). (p. 590.)

DASYCROTAPHA Tweedd.

(Sharpe, Cat. Bds., VII, p. 5\%4, 1883.)

speciosa Twcedd. (p. 574.) 
ZOSTERORNIS Grunt.

(Grant, Bull. B. O. C., III, p. i, 1894.)

striata Grunt, l. c., p. ii (1894); id. Lbis, Luzon 1895 , pl. iv, fig. 1.

whiteheadi Grant, l. c., p. i (1894); id. lbis, Luzon 1894 , pl. $x v$, fig. 1 .

pygmæa Grant, Bull. B. O. C., vi, p. xviii Leyte Samar (1896) ; id. 1bis, 1897 , pl. vi, fig. 1.

plateni (W. Blusius), J. f. O., 1890, p. 147. Mindanao

capitalis (Trueceld.). (p. 582.) Basilan Mindanao

Dinagat Panaon

Leyte

nigrocapitata (Steere), List. Jils. \& Mans. Exp. Philipp., p. 17 (1890).

dennistouni Grant, Bull. 13. O. C., V, p. ii Luzon (1895) ; id. Ibis, 1896, pl. iii, fig. 2.

MIXORNIS Hodgs.

(Sharpe, Cat. Bds., VH, p. 575, 1883.)

woodi Sharpe. (p. 577.)

Balabac Palawan

cagayanensis Guillem., P. Z. S., 188.,, p. 419 , pl. xxv.

Cagayan Sulu

MACRONUS Jard. \& Selby.

(Sharpe, Cat. Bds., VII, p. 583, 1883.)

striaticeps Sharpe. (p. 584.)
$\begin{aligned} & \text { mindanensis Steere, List. Bds. \& Mams. } \\ & \text { Steere Exp., p. } 17(1890) .\end{aligned}$
$\begin{aligned} & \text { Dinagat } \\ & \text { Leyte } \\ & \text { XVIII, p. } 4 \text { (1905). }\end{aligned}$

${ }^{1}$ A single specimen of Zosterornis, obtained at Mariveles, in the Province of Bataan, Jsland of Iuzon, can not be differentiated from $Z$. nigroeapitata heretofore recorded from Samar and Leyte without specimens for actual comparison. While it may be ultimately proven to belong to an undescribed species, it has been provisionally assigned by MeGregor to $Z$. nigroeapitata [Govt. Jab. Pub]. No. 34, p. 29 (1905) ]. 
Subfamily BRACHYPTERYGINÆ.

BRACHYPTERYX Horsf.

(Sharpe, Cat. Bds., VII, p. 25, 1883.) [Callene, pt., Sharpe, t. c. 15. Cf. Oates, Faun. Brit. Ind., Birds, I, p. 185.]

brunneiceps Grant, Ibis, 1896, p. 547.

poliogyna Grant, Bull. B. O. C., IV, p. xl Luzon

Mindoro . $(1895)$; id. Ibis, pl. xii, fig. 1.

mindanensis Mearris, Proc. Biol. Soc. Wash., XVIII, p. 3 (1905).

Mindanao

LEONARDINA Mearns.

(Leonardia Mearns, 1905, nec Tapparone-Canefri (1890).)

[Cf. Mearns, Proc: Biol. Soc. Wash., XVIII, p. 88 (1905).]

woodi Mearns, Proc. Biol. Sòc. Wash., XVIII, p. 2 (1905).

Mindanao

Family TURDIDAE.

Subfamily TURDIN平.

MERULA Leach.

(Seebohm, Cat. Bds., V, p. 232, 1881.)

nigrorum (Grant), Ibis, 1896, p. 544.

Negros

thomassoni Seebohm, Bull. B. O. C., III, p.

Luzon li (1894).

mindorensis (Grant), Ibis, 1896, p. 465.

Mindoro

kelleri Mearns, Proc. Biol. Soc. Wash., XVIII, p. 6 (1905). 
GEOCICHLA Temm.

(Seebohm, Cat. Bds., $\mathrm{T}$, p. $11 \%$, 1881.)

interpres (Temm.). (p. 166.)

[avensis Gray (p. 167). Cf. Oates, Fauma Brit. India II, p. 138, 1890.]

Malay Peninsula

Northwestern Borneo

Sumatra Java

Lombok Sumbawa

Baslian Tawi Tawi

Sulu

cinerea Boums \& Worcester, Oce. Papers Mindoro

Minnesota Acad., I, No. 1, p. 23 (1894).

OREOCICHLA Gould.

(Geocichla pt. Seebohm, Cat. Bds., V, p. 14\%, 1881.)

[Cf. Seebohm, Monogr. 'Turdidx, I, pp. 1-32.]

varia (Pall.). (p. 151.)

[hancei Swinh. (p. 153.)]

Japan

China (winter)

Burma (winter)

Accidental In Europe

Western and eastern Siberia

Luzon

TURDUS Linn.

(Seebohm, Cat. Bds., V, p. 184, 1881.)

pallidus $G m$. (p. 274.)

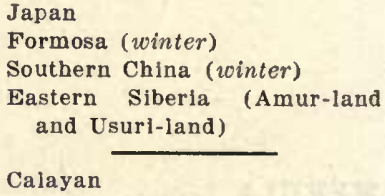

chrysolaus T'cmm. (p. 275.)

China (winter)

Formosa (winter)

Japan Hainan

Eastern Siberia (Ussuri-land, Sakhalin)

Calayan Luzon

obscurus $G m$. (p. 273.)

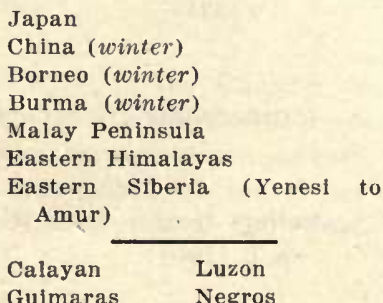


PETROPHILA Swains.

(Monticola pt. Seebohm, Cat. Bds., $\mathrm{V}$, p. 312.)

$\lceil C f$. Oates, Fauna Brit. India, Bds., I, p. 142, 1889.]

manilla Bodd.

[solitaria (P. L. S. Miill., nec Linn.). (p. 319.)] [teste Stejneger in litt.]

\begin{tabular}{ll} 
Japan \\
Eastern Siberia \\
Soutbern China (winter) \\
Malay Archipelago (winter) \\
Burmese Provinces (winter) \\
\cline { 2 - 2 } & \\
Agutaya & Negros \\
Balabac & Palawan \\
Basilan & Panaon \\
Cagayancilio & Panay \\
Calamianes & Rombion \\
Calayan & Sibay \\
Cuyo & Sibuyan \\
Guimaras & Siquijor \\
Leyte & Suiu \\
Lubang & Tablas \\
Luzon & Tawi Tawi \\
Masbate & Ticao \\
Mindanao & Verde \\
Mindoro & \\
&
\end{tabular}

Subfamily RUTICILLINE.

CHIMARRHORNIS Hodgs.

(Sharpe, Cat. Bds., VII, p. 4\%, 1883.)

bicolor Grant, Bull. B. O. C., III, p. 44 Luzon

(1894); idl. Ilis, 1894, pl. xv, fig. 2.

CALLIOPE Gould.

(Erithachus pt. Seebohm, Cat. Bds.,

V, p. 293, 1881.)

[Cf. Oates, Faun. Brit. Ind., Birds, I, p. 101, 1889.]

calliope (Pall.). (p. 305.)

Europe (accidental)

Southern China (winter)

Burmese provinces (winter)

Siberia Northern China

Northern and Central India (winter)

$\begin{array}{ll}\text { Calayan } & \text { Mindoro } \\ \text { Iuzon } & \text { Negros } \\ \text { Masbate } & \end{array}$


COPSYCHUS Wagler.

(Sharpe, Cat. Bds., VII, p. 60, 1883.)

mindanensis $(G m$.$) . (p. 60.)$

$\begin{array}{ll}\text { Basilan } & \text { Romblon } \\ \text { Bongao } & \text { Samar } \\ \text { Cebu } & \text { Semirara } ?^{1} \\ \text { Guimaras } & \text { Sibuyan } \\ \text { Leyte } & \text { Siquijor } \\ \text { Lubang } & \text { Sulu } \\ \text { Luzon } & \text { Tablas } \\ \text { Masbate } & \text { Tawi Tawi } \\ \text { Mindanao } & \text { Ticao } \\ \text { Mindoro } & \text { Verde } \\ \text { Negros } & \end{array}$

CITTOCINCLA, Sct.

(Sharpe, Cat. Bds., VII, p. $8 \pm$, 1883.)

[Cf. Hartert. Nov. Zool. ix, pp. $572-573,1892$.

nigra Sharpe. (p. 90.)

Balabac Palawan

Calamianes

Catanduanes Marinduque

luzoniensis (Kittl.). (p. 91.)

Luzon

Cebu

cebuensis Steere, List. Bds. \& Mams. Steere Exp., p. 20 (1890).

superciliaris Bourns of Worcester, Oce. Pa-

Masbate

Ticao pers Minnesota Acad., I, No. 1, p. 23 (1894).

nigrorum Grant, Ibis, 1896, p. 547.

Negros

PRATINCOLA Koch.

(Sharpe, Cat. Bds., IV, p. 178, 1879.)

caprata (Linn.). (p. 195.)

\begin{tabular}{ll}
$\begin{array}{l}\text { Persia } \\
\text { Indian Peninsula } \\
\text { Burmese provinces } \\
\text { Java }\end{array}$ \\
\cline { 2 - 2 } $\begin{array}{l}\text { Bohol } \\
\text { Bebu }\end{array}$ \\
$\begin{array}{ll}\text { Lubang } \\
\text { Luzon } \\
\text { Masbate }\end{array}$ & $\begin{array}{l}\text { Mindoro } \\
\text { Negros }\end{array}$ \\
& Panay \\
Siquijor
\end{tabular}

SAXICOLA Bechst.

(Seebohm, Cat. Bds., V, p. 362, 1881.)

œnanthe (Linn.). (p. 391.)

[Cf. Stejneger, Pr. U. S. Nat. Mus., XXIII, p. 473 (1901); id. Auk, XVIII, p. 186 (1901).]

[cenanthoides Vig.]

Europe and northern Asia to Alaska

Indian Peninsula and eastern Africa (winter)

Calayan

${ }^{1}$ A bird seen by me on Semirara in April, 1905, but not killed, was in all probability $C$. mindanensis.-Worces'TER. 
Family SYLVIIDE.

LOCUSTELLA Kaup.

(Seebohm, Cat. Bds., V, p. $10 \%$, 1881.)

fasciolata (Gray). (p. 109.)

$[C f$. A. B. Meyer \& Wiglesw., B. Celebes, p. $524(1898)$.

Talaut Islands

Sangir Islands

Eastern Siberia

Molucca Islands

China

Japan

Batchian, Halmahera, Morotai (winter)

$\begin{array}{ll}\text { Calayan } & \text { Sulu } \\ \text { Luzon } & \text { Tablas } \\ \text { Marinduque } & \text { Tawi Tawi }\end{array}$

ochotensis (Midd.). (p. 113).

[dybowskii Ridgw., Pr. U. S. Nat. Mus., VI, p. 92 (1883).]

Kurile Islands

Borneo (winter)

Northeastern Siberia

Greater Sunda Islands

Kamtchatka Japan

Calayan Mindoro

Marinduque Romblon

lanceolata (Temm.). (p. 118.)

China (winter)

Eastern Europe (Russia)

Andaman Islands (winter)

Indian Peninsula (winter)

Burmese provinces (winter)

Siberia

Central Asia

Calayan

Luzon

\section{ACROCEPHALUS NaUm.}

(Seebohm, Cat. Bds., V, p. 8\%, 1881.) 「Tatare Lesson; Sharpe, Cat.

Bds., VII, p. 524 (1883).

Cf. Zool. Rec., Aves, 1883,

p. 26 ; Büttik. Notes Leyden Mus., XIV, p. 13 (1892).]

sorgophilus (Swinh.). (p. 94.)

China

Luzon

orientalis (Temm. \& Schl.). (p. 97.) 
TRIBURA IIodgs.

( Lusciniola pt. Seebohm, Cat. Bds.,

V, p. 120, 1881.)

[Cf. Oates, Faun. Brit. Ind., Birds, I, 361, 1889.]

seebohmi (Grant), Bull. B. O. C., IV, p. xl

Luzon

(1895) ; icl. Ibis, 1895 , p. 443.

ORTHOTOMUS Horsf.

(Sharpe, Cat. Bds., VII, p. 219

(1883).

[Cf. Grant, Ibis, 189\%, p. 229.]

frontalis Sharpe. (p. 220.)

Baslan

Bohol

Leyte

Dinagat

Mindanao

Samar

cinereiceps Sharpe. (p. 222.)

Basilan

Mindanao

nigriceps Tweedd. (p. 222.)

Mindanao

samarensis Steere, List Bds. \& Mams. Steere

Leyte

Samar

Exp., p. 20 (1890.)

castaneiceps Wald. (p. 223.)

[panayensis Stcere, List. Bds. \& Mams. Steere Exp., p. 20, 1890. Cf. Grant,

Ibis, 1896, p. 549.]

derbianus Moore. (p. 224.)

Luzon

chloronotus Grant, Bull. B. O. C., III, p. ii Luzon (1895).

ruficeps (Less.). (p. 224.)

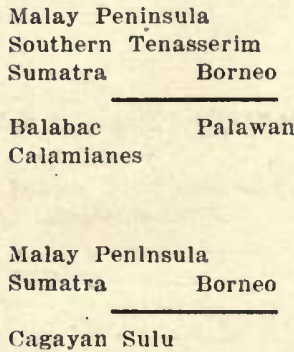

cineraceus $B l y l h$. (p. 225.) 
CISTICOLA Kaup.

(Sharpe, Cat. Bds., VII, p. 235, 1883.)

cisticola (Temm.). (p. 259.)

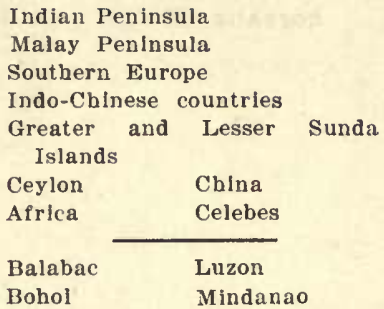

exilis (Vig. \& Horsf.). (p. 269.)

Southern China

[oryziola S. Miill. (p. 240, note.) Cf. Sharpe, Notes Leyden Mus., VI, p. 168.]

Indian Peninsula

Burmese provinces

Malay Peninsula and Archipelago

[phragmitoides Kuhl. (p. 185, note), teste Finsch in litt.]

[Cf. also Oates, Faun. Brit. Ind., Birds, I, pp. 371-373, 1889.]

\begin{tabular}{ll} 
Australla & Formosa \\
\cline { 2 - 2 } Calamianes & Panay \\
Calayan & Romblon \\
Cebu & Samar \\
Fuga & Semlrara \\
Lubang & Slbay \\
Luzon & Slbuyan \\
Marinduque & Slquljor \\
Masbate & Sulu \\
Mindanao & Tablas \\
MIndoro & Ticao \\
Negros & Verde
\end{tabular}

MEGALURUS Horsf.

(Sharpe, Cat. Bds., VII, p. 122, 1883.)

palustris Horsf. (p. 123.)

Manipur

[punctalus De Vis, Ibis, 1897, p. 385.

=macrurus, juv. Rotsch. \& Hartert. Ornn. MB. vii, p. 139, 1898.]

Burmese provinces

Soutbeastern New Guinea

Northern and Central Indla to Bbutun and Buxa Doars

\begin{tabular}{ll} 
Assam & Java \\
\cline { 2 - 2 } Catanduanes & Mindana \\
Luzon & Mindoro \\
Marlnduque & Samar \\
Masbate & Tleao
\end{tabular}

ruficeps Treedd. (p. 125.)

$\begin{array}{ll}\text { Basilan } & \text { Mindoro } \\ \text { Bobol } & \text { Negros } \\ \text { Cebu } & \text { Panay } \\ \text { Guimaras } & \text { Romblon } \\ \text { Lubang } & \text { Samar } \\ \text { Luzon } & \text { Slbuyan } \\ \text { Marlnduque } & \text { Slquijor } \\ \text { Masbate } & \text { Tablas } \\ \text { Mindanao } & \text { Ticao }\end{array}$


ACANTHOPNEUSTE Blas.

(Phylloscopus pt. Seebohm, Cat. Bds., V, p. 3\%, 1881.)

borealis (Blas.). (p. 40.)

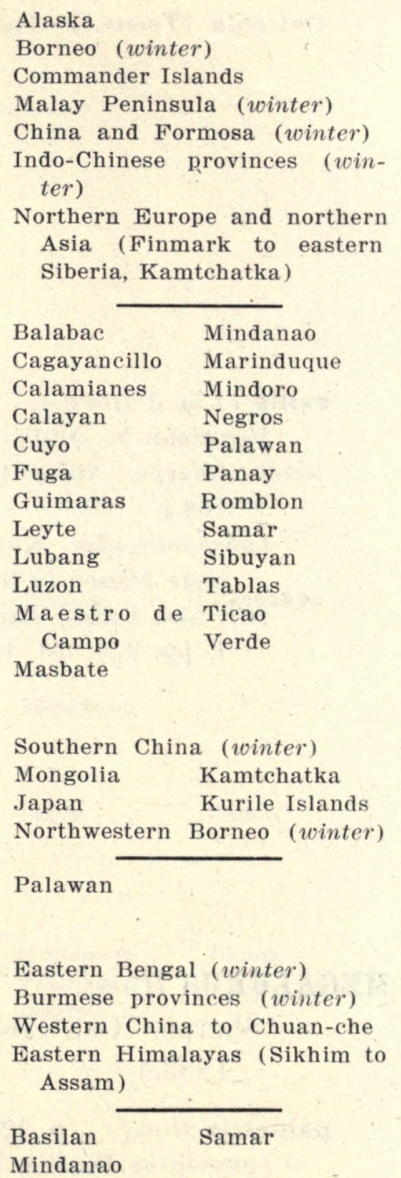

lugubris $(B l y t h) . \quad$ (p. 48.)

Northern Luzon

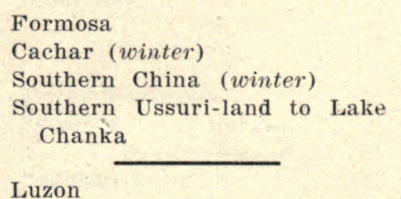

HORORNIS Hodgs.

(Cettia pt. Seebohm, Cat. Bds., V, p. 133, 1881.)

[Cf. Oates, Faun. Brit. Ind., Birds, I, p. 434, 1889.]

seebohmi (Grant), Ibis, 1894, p. 507.

canturiens (Swinh.). (p. 141.) 
minuta (Svinh.). (p. 141.)

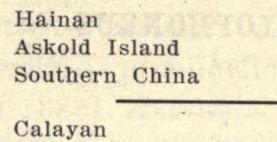

PHYLLERGATES Sharpe.

(Shärpe, Cat. Bds., VII, p. 229, 1883;

cf. Zool. Rec., 1883, Aves, p. 25, note.)

[Phyllobates Oates, Bds. Brit. Burm., I, p. 110 (1883).]

philippinus Hartert, Nov. Zool., IV, p. 517

Luzon

(1897.)

heterolæmus Mearns, Proc. Biol. Soc. Wash., XVIII, p. 86 (1905).

Family ARTAMIDE.

ARTAMUS Vieill.

(Sharpe, Cat. Bds., XIII, p. 2, 1890.)

leucorhynchus (Linn.), teste Richmond.

Australia

[leucogaster (Valenc.). (p. 3.)]

Papuan Islands

Andaman Islands

Malay Archipelago

Basilan Negros

Bohol Palawan

Bongao Panay

Calamianes Romblon

Catanduanes Samar

Cebu Semirara

Cuyo Sibay

Guimaras Sibuyan

Leyte Siquijor

Lubang Sulu

Luzon Tablas

Marinduque Tawi Tawi

Masbate Ticao

Mindanao Verde

Mindoro

Family LANIIDAE.

Subfamily LANIIN E.

ENNEOCTONUS Boie.

(Lanius pt. Gadow, Cat. Bds., VII, p. 232, 1883; Grant, Nov. Zool., IX, p. 449, 1902.)

tigrinus (Drap.). (p. 289.)

Borneo

Malay Peninsula

Sumatra

Java

Korea

China

Sulu 
CEPHALOPHONEUS Fitz.

(Ianius pt. Gadow, Cat. Bds., VIII, p. 232, 1883; Grant, Nov. Zool., IX, p. 449, 1902.)

validirostris (Grant), Bull. B. O. C., 1II, Luzon Mindoro p. xlix, 1894).

nasutus (Scop.).

[cephalomelas Bp. (]. 269.)]

[schalowi Gadow.]

\begin{tabular}{ll} 
Northern Borneo \\
\cline { 2 - 2 } Bohol & Mindanao \\
Calamianes & Mindoro \\
Cebu & Negros \\
Guimaras & Panay \\
Leyte & Samar \\
Luzon & Siquijor \\
Masbate &
\end{tabular}

suluensis Mearns, Proe. Biol. Soc. Wash., Sulu XVIII, p. 86 (1905).

OTOMELA, $B p$.

(Lanius pt. Gadow, Cat. Bds., VIII, p. 232, 1883; Grant, Nov. Zool., IX, p. $449,1902$.

cristata $^{1}$ (Linn.). (p. 271.)

Borneo

Eastern Siberla

Malay Peninsula

Indian Peninsula

Indo-Chlnese provinces

Philipplne Islands (winter)

lucionensis (Linn.). (p. 274.)

\begin{tabular}{ll} 
Mongolla & \\
Northern China \\
Malay Archipelago \\
Southern India (winter) \\
Andaman and Nlcobar Islands \\
Korea & \multicolumn{1}{c}{ Formosa } \\
\cline { 2 - 2 } Agutaya & Masbate \\
Baiabac & Mindanao \\
Basilan & Mindoro \\
Bohol & Negros \\
Cagayancillo & Palawan \\
Calamianes & Panaon \\
Calayan & Panay \\
Camiguln & Romblon \\
Catanduanes & Samar \\
Cebu & Sibay \\
Cuyo & Sibuyan \\
Fuga & Siquijor \\
Guimaras & Sulu \\
Leyte & Tablas \\
Lubang & Tawl Tawi \\
Luzon & Ticao \\
Maestro de & Verde \\
Campo & \\
\hline
\end{tabular}

${ }^{1}$ The two shrikes, $O$. cristata and $O$. superciliosa, are included in this list on the authority of Sharpe's Hand-List. 'Their distribution within the Philippines is not known to me.-MCGREGor. 
superciliosa $^{1}$ (Lath.). (p. 273.)

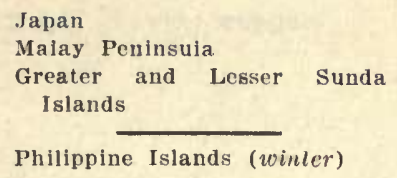

Subfamily PACHYCEPALIN E.

HYLOTERPE Cab.

(Pachycephala pt. Gadow, Cat. Bds., VIII, p. 182, 1883.)

philippinensis Walden. (p. 221.)

Basllan

Mindanao

Dinagat

Leyte

Luzon

Samar

Siquijor

apoensis Mearns, Proc. Biol. Soc. Wash.,

Mlndanao

XVIII, p. 86 (1905).

homeyeri Blasius, J. f. O., 1890, p. 141.

Bongao

Sulu

Sibutu

Tawl Tawl

whiteheadi Sharpe, Ibis, 188s, p. 198.

Palawan

[plateni W. Blasius, Ornis, 1888, p. 311.]

winchelli Bourns of Worester, Occ. Papers

Minnesota Acad., I, No. 1, p. 21 (1894).

[major Bourns \& Worcester, 1. c. p. 22.]

Masbate

Sibuyan

Negros

Tablas

Panay

Ticao

mindorensis Bourns \& Worcester, $t$, c. p. $22 . \quad$ Mindoro

albiventris Grant, Bull. B. O. C., III, p. xlix Luzon

Mindoro

(1894).

fallax McGregor, Bull. Philippine Mus., No. 4, p. 27 (1904).

Calayan

\section{Family PARIDAE. \\ Subfamily PARINE.}

PARDALIPARUS Selys-Longch.

(Selys-Longehamps, Bull. S. \%.

France IX, p. 73, 1884; Branchi,

Ann. Mus. St. Petersb., VII, p.

24\%, 1902.)

(Parus pt. Gadow, Cat. Bds...

VIII, p. 3, 1883.)

[Hellm., Tierr., Parida, p. 80. 1901. $]$

amabilis (Sharpe). (p. 22.) 
elegans (Less.). (1).2.2.)

$\begin{array}{ll}\text { liongao } & \text { Mindoro } \\ \text { Calayan } & \text { Negros } \\ \text { Cebu } & \text { Panay } \\ \text { Guimaras } & \text { Sulu } \\ \text { Luzon } & \text { Tawi Tawi } \\ \text { Masbate } & \text { Ticao }\end{array}$

mindanensis Mearns. Proc. Biol. Soc. Waslı., Mindanao XV111, p. 8 (1905).

PENTHORNIS Hellmayr.

(Hellmayr, J. f. O. 1901, p. 163:

Tierreich, Parida, p. 32, 1903.)

(Parus pt. Gadow, Cat. Bds., VIII, p. 3, 1883.)

semilarvatus (Salvadori). (p. 38.)

Luzon Negros

[Cf. Wardlaw Ramsay, lbis, 1884, p. 334.1

Iuzoniensis ( $(\mathrm{m} m$.$) .$

[Micropus nehrliorni ]3lasius, J. f. O., 1890. 1. 147. ('f. Sllatpe, Bull. B. O. (.. IV, p. ii (1894) : Hellmayr, J. f. O., 1901, pp. 171, 172.]

\section{Family SITTIDAE.}

CALIISITTA Reichenb.

(Dendroplita Swains.; Sitta pt. Gadow, Cat. Bds., VIII, p. 341, 1883; Hellm., Tierr., Sittida, p. 191, 1903.)

frontalis (Swains.). (p. 358.)

Southern India

Malay Peninsula

Burmese provinces

Ceylon Java

Himalayas (Kumaon to Assam)

Balabac Palawan

œnochlamys (s'harpe). (1. 359.)

Basilau Luzon

Cebu Mindanao

Gulmaras Negros

Leyte Panay

mesoleuca (Grant), Bull. B. O. C., 11I, Luzon p. xlix (1894).

lilacea (Wlitehead), Bull. B. O. C., VI, Basilan, Samar p. xlix (1897). 


\section{Fanily CERTHIIDE.}

RHABDORNIS Reichenb.

(Climacteris pt. Gadow, Cat. Bds.,

VIII, p. 333, 1883.)

(Cf. Grant, Ibis, 189\%, p. 235.)

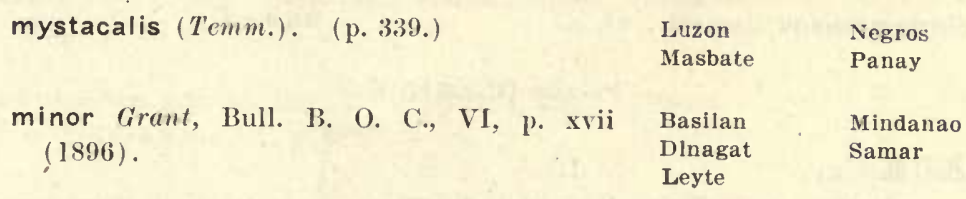

inornata Grant, t. c. p. xviii; ill. lbis, 1897, Samar pl. vi, fig. 2.

Family ZOSTEROPIDE.

ZOSTEROPS Vig. and Horsf.

(Gadow, Cat. Bds., IX, p. 146, 1884.)

everetti T'ueedd. (p. 163.) Cebu

nigrorum Tweedl. (p. 186.)

Cresta de Gallo Pauay

Masbate Ticao

Negros

siquijorensis Bourns and Worcester, Occ.

Negros

Siquijor

Papers Minnesota Acad., 1, No. 1, p. 21 (1894).

Iuzonica Grant, Bull. B. O. C., No. XXIV, p. xxii (1895).

aureiloris Grant, Bull. B. O. C., No. XXVIII, - p. xl (1895).

basilanica stcere, List. Bds. and Mans. Steere Exp., p. 21 (1890).

Basllan

Bongao

Dinagat

Sama

Leyte

Mlindoro

richmondi I/clireyor, Proe. Biol. Soc. Waslı., XV11, p. 165 (1904).

[flarissima MeGregor, Bull. Philippine: Mus., No. 4, p. 26 (1904) nec Hartert.]

meyeni Bonap. (p. 180.)

[Cf. Grant, lbis, 189.5, p. 452.]

Cagayanclllo

whiteheadi Hartert, Bull. B. O. C., XIV, No. C, p. 13 (1903).

vulcani (Hartert), Bull. B. O. C., XIV, No. C, Mindanao p. 14 (1903). 
goodfellowi Hartert, Bull. B. O. C., XIV, Ilndanao No. C, p. 13 (1903).

HYPOCRYPTADIUS Hertert.

(Hartert, Bull. B. O. C. XIV, No. C, p. 13, 1903.)

cinnamomeus Hartert. (I. c.) Mindaua

Family DICAEIDAE.

DICEEU Cuv.

(Sharpe, Cat. Bds.. X, p. 10, 1885.)

retrocinctum Gould. (p. 35.)

Luzon

Mindoro

hæmatostictum sharpe. (p. 3.).)

Guimaras

Panay

papuense $\left(\right.$ Gmel.)..$^{1} \quad$ (p. 36.)

Negros

Basilan Mariuduque

Cebu Masbate

Dlnagat Mindanao

Leyte Samar

Lubang Ticao

Luzon Verde

Iuzoniense Grant, Bull. B. O. C., III, p. 1. Luzon (1894).

ap.o Hartert, Bull. B. O. C., X1V, No. CVII, Mindanao p. $79(1904)$.

bonga Hartert, t. c. p. 80 .

Samar

davao Mearns, Proc. Biol. Soc. Wash., XVIII,

Mindanao p. 87 (1905).

flaviventer Meyer, J. für O., 1894, p. 91, ex

Cebu

Dubois, Syn. Av., p. 679.

dorsale Sharpe. (p. 40.)

Masbate Panay

Negros

pallidior Boums and Worecster, Occ. Papers. Cebu

Minnesota Acad. Sei., I, No. 1, p. 18 (1894).

sibuyanicum Bourns and Worcester. (1. c.) Sibuyan

intermedium Bourns and Worcester. (t. c. Romblon p. 19.)

assimilis Bourns and Worcester. (1. c.) Sulu Tawi Tawi

${ }^{1}$ This species is usually called Dicaeum rubriventer Lesson, Gmelin's name Pipra papuensis being misleading.-McGregor. 


\begin{tabular}{|c|c|c|}
\hline xanthopygium 'T'wcedd. (p. 40.) & $\begin{array}{l}\text { Luzon. } \\
\text { Marinduque }\end{array}$ & Mindoro \\
\hline cinereigulare Twecdd. (p. 40.) & $\begin{array}{l}\text { Leyte } \\
\text { Mindanao }\end{array}$ & Samar \\
\hline $\begin{array}{l}\text { besti Steere, List. Bds. and Mams. Steere } \\
\text { Lixp., p. } 22(1890) \text {. }\end{array}$ & Siquijor & \\
\hline $\begin{array}{l}\text { sibutuense Sharpe, Ibis, 1894, pp. 122, } 251 \text {; } \\
\text { ex Dubois Syn. Av., p. } 679 .\end{array}$ & Sibutu & \\
\hline pygmæum (Kittlitz). (p. 43.) & $\begin{array}{l}\text { Balabac } \\
\text { Calayan } \\
\text { Fuga } \\
\text { Guimaras } \\
\text { Leyte } \\
\text { Lubang } \\
\text { Luzon } \\
\text { Maestro de } \\
\quad \text { Campo } \\
\text { Masbate }\end{array}$ & $\begin{array}{l}\text { Mindoro } \\
\text { Negros } \\
\text { Paiawan } \\
\text { Romblon } \\
\text { Samar } \\
\text { Semirara } \\
\text { Sibuyan } \\
\text { Siquijor } \\
\text { Ticao }\end{array}$ \\
\hline
\end{tabular}

nigrilore Hartert, Bull. B. O. C., $\mathrm{XV}$, No. Mindanao CIX, p. 8 (1904).

hypoleucum Sharpe. (p. 37.)

Basilan Stasi

Bongao . Suiu

mindanense Tweedd. (p. 37.)

Basilan Sulu

Mindanao Tawi Tawi

everetti Tweedd. (p. 47.)

Dinagat Panaou

Leyte Samar

obscurum Grant, Bull. B. O. C., III, p. 1 Luzon (1894).

\section{PRIONOCHILUS Stricli.}

(Sharpe, Cat. Bds., X, p. 63 pt., 1885.)

johannæ Sharpe, Ibis, 1888, p. 201.

quidricolor T'weedd. P. Z. S., 1877, p. 762.

olivaceus Tweedd. (p. 75.)

bicolor Bourns and Worcester, Occ. Papers Minnesota Acad., I, No. 1, p. 20 (1894).

inexpectatus Hartert, Nov. Zool., II, pp. 64, 486 (1895).

$35916-7$
Balabac

Busuanga

Cebu

Baslian

Dinagat

Leyte

Minđanao

Samar

Mindanao

Leyte

Luzon

Mindoro
Negros

Samar 
PIPRISOMA Blyth.

(Sharpe, Cat. Bds., X, p. 63, pt. 1885.)

æruginosum (Bourns and Woreester), Occ.

Papers Minnesota Acad., I, No. 1, p. 20

(1894).

$\begin{array}{ll}\text { Cebu } & \text { Mindoro } \\ \text { Lubang } & \text { Romblon } \\ \text { Luzon } & \text { Sibuyan } \\ \text { Mindanao } & \end{array}$

Family NECTARINIIDE.

CHALCOSTETHA $\mathrm{Cab}$.

(Gadow, Cat. Bds., IX, p. 12, 1884.)

insignis (Jard.). (p. 12.)

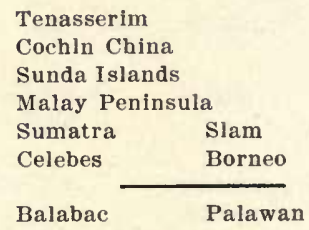

ETHOPYGA $\mathrm{Cab}$.

(Gadow, Cat. Bds., IX, p. 13, 188t.)

$\begin{array}{llll}\text { magnifica Sharpe. (p. 24.) } & \text { Cebu } & \text { Sibuyan } \\ & \text { Negros } & \text { Tablas } \\ \text { Panay } & \end{array}$

boltoni Mearns, Bull. Biol. Soe. Wash., XVIl1,

Mindanao

p. 4 (1905).

shelleyi sharpe. (p. 29.)

Balabac

Palawan

Calamianes

Mindanao Samar

bella T'uecud. (p. 29.)

Tawi Tawi

arolasi Bourns and Woreester, Oce. Paper's,

Sulu

Minuesota Acad., I, No. 1, p. 17 (1894).

bonita Bourns and Worester. (1. c.

Cebu Panay

Masbate Tícao

flavipectus Grant, Bull. B. O. C., III, p. xlix

Luzou

Mindoro

(1894).

[minula Bourns and Worcester, Occ. Papers Minnesota Acad., I, No. 1, p. 18 (1894).]

rubrinota MeGregor, Govt. Lab. Publ. No. 25,

Lubang p. $30(1905)$. 
EUDREPANIS Sharpe.

(Gadow, Cat. Bds., IX, p. 13 pt. 1884.)

pulcherrima (Sharpe). (p. 31.)

Basilan Leyto

Dinagat Samar

jefferyi Grant, Bull. B. O. C., III, p. 1 (1994). Luzon

CINNYRIS Cuv.

(Gadow, Cat. Bds., IX, p. 31, 1884.)

sperata (Linn.). (p.63.)

$\begin{array}{ll}\text { Calamianes } & \text { Negros } \\ \text { Cebu } & \text { Nipa } \\ \text { Dinagat } & \text { Palawan } \\ \text { Guimaras } & \text { Panay } \\ \text { Leyte } & \text { Rombion } \\ \text { Lubang } & \text { Samar } \\ \text { Luzon } & \text { Sibuyan } \\ \text { Marinduque } & \text { Siquljor } \\ \text { Masbate } & \text { Tabias } \\ \text { Mindanao } & \text { Ticao } \\ \text { Mindoro } & \end{array}$

whiteheadi Grant, Bull. B. O. C., 11, p. 1 (1894).

Caiayan

Luzon

Fuga

juliæ Tweedd. (p. 64.)

Baslian

Maianipa

Tawi Tawi

Mindanao

Luzon

Catanduancs

flagrans (Oust.). (p. 88.)

[excellens Grant, Bull. B. O. C., No. XXIII, p. xviii (1895); cf. Whitehead, Ibis, 1899, p. 230.]

guimarasensis Stcere, List. Bds. and Mams. Steere Exp., p. 22 (1890).

jugularis (Limn.). (p. 84.)

[dinagutensis Mearns, ${ }^{1}$ Proc. Biol. Soc. Wash., XVIII, p. 5 (1905). obscurior Grant, Bull. B. O. C., III, p. 1 (1894); ef. Grant, Ibis, 1895, p. 451.]

aurora (Tweedd.). (p. 88.)

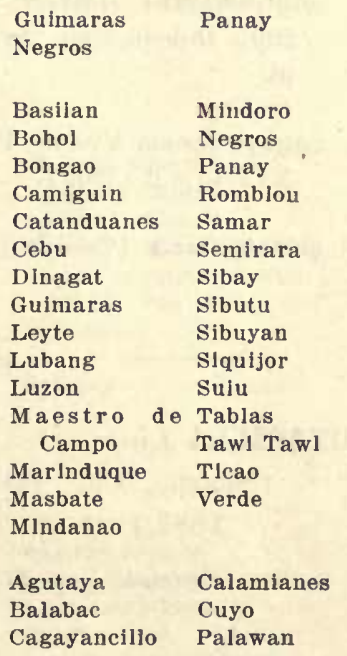

${ }^{1}$ In view of the great variation of color on the breast of Cinnyris aurora and on that of $C$. jugularis we do not recognize $C$. dinagatensis as valid. The latter name is confessedly applied to a spccimen intermediate between the two older species.-MCGREGOR AND WORCESTER. 
ARACHNOTHERA T'mm.

(Gadow, Cat. Bds., IX, p. 100, 1884.)

flammifera Tweedd. (p. 104.)

Basilan Mindanao

Leyte Samar

dilutior Sharpe. (p. 105.)

Palawan

philippinensis (Steere), List. Bds. and

Leyte

Samar

Mams. Steere Exp., p. 21 (1890).

Mindanao

ANTHREPTES Swains.

(Gadow, Cat. Bds., IX, p. 112, 1884.)

malaccensis (Scop.). (p. 122.)

\begin{tabular}{ll} 
Malay Peninsula \\
Siam & $\begin{array}{l}\text { Java } \\
\text { Sumatra }\end{array}$ \\
Cochin China & $\begin{array}{l}\text { Borneo } \\
\text { Flores }\end{array}$ \\
\cline { 2 - 2 } Balabac & Palawan \\
Bongao & Sibutu \\
Calamlanes & Tawi Tawl
\end{tabular}

rhodolæma Shelley. (p. 122, pt.)

Borneo

Palawan

chlorigaster Sharpe. (p. 122, pt.)

$\begin{array}{ll}\text { Basilan } & \text { Panay } \\ \text { Lubang } & \text { Romblon } \\ \text { Cebu } & \text { Sibuyan } \\ \text { Masbate } & \text { Tablas } \\ \text { Mindanao } & \text { Tawi Tawi } \\ \text { Negros } & \text { Ticao }\end{array}$

wiglesworthi Hartert. Nov. Zool., IX, p. Sulu 209; Dubois Syn. Av., 1113, chlorigaster, pt.

cagayanensis Mearns, Proc. Biol. Soc. Wash., Cagayan Sulu XVIII, p. 6 (1905).

griseigularis (Tweedd.). (p. 126.)

$\begin{array}{ll}\text { Luzon } & \text { Sakuijoc } \\ \text { Mindanao } & \text { Samar } \\ \text { Mindoro } & \end{array}$

\section{Family MOTACILLIDAE.}

MOTACILIA Linn.

(Sharpe, Cat. Bds., X, p. 45\%, pt., 1885.)

ocularis Swinh. (p. 471.)

Burma (winter)

Aleutian Islands ?

Northeastern Siberia

China Kamchatka

Lower California (accidental)

Balabac Luzon

Calayan Palawan

Lubang 
melanope Pall. (p. 497.)

flava Linn. See Page 110.

BUDYTES Cuv.

(Sharpe, Cat. Bds., X, p. $45 \%$ pt., 1885.)

leucostriatus Hom. (p. 516, pt.)

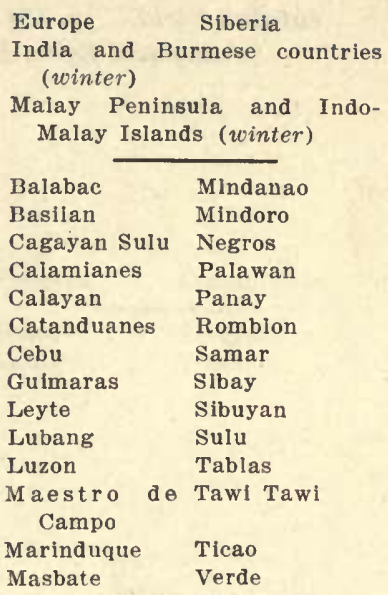

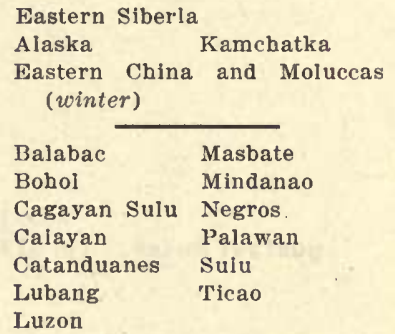

\section{IIMONDROMUS Gould.}

(Sharpe, Cat. Bds., X, p. 532, 1885.) indicus $(\mathrm{Gm}$.$) . (1. c.)$

\section{Eastern Siberia \\ Northern China \\ Indian Peninsuia \\ Ceylon, Andaman Islands, Bur- mese countries, Cocbln CbIna, Maiay Peninsula, and Java (winter) \\ Balabac \\ Calayan}

ANTHUS Bechst.

(Sharpe, Cat. Bds., X, p. 534, 1885.) maculatus Hodgs. (p. 547.)

\author{
Eastern Siberia \\ Indian Peninsula \\ Loo Choo Islands \\ China Japan \\ Indo-Burmese countrles \\ Calayan Palawan \\ Luzon
}


rufulus Vieill. (p. 574.)

[malayensis Eyton.] cervinus (Pall.). (p. 585.)

gustavi Swinh. (p. 613.)
Malay Peninsula

Burmese countries

Africa countries

Ceylon Java

Sumatra Borneo

Bohol Mindoro

Calamianes Negros

Calayan Palawan

Cebu Panaon

Fuga Panay

Guimaras Romblon

Leyte Samar

Lubang Semirara

Luzon Sibuyan

Maestro de Siquijor

Campo Tablas

Masbate Ticao

Mindanao

Kurile Islands

Borneo (winter)

$\begin{array}{ll}\text { Alaska } & \text { Asia } \\ \text { England } & \text { Europe }\end{array}$

Northern Africa (winter)

Lower California (accidental)

Formosa and Hainan (winter)

Persia and northwestern India (winter)

China and Indo-Burmese countries (winter)

$\begin{array}{ll}\text { Calayan } & \text { Mindanao } \\ \text { Luzon } & \text { Palawan }\end{array}$

Siberia Borneo
Celebes Kamtchatka
Timor (winter)
China (migration)
Moluccas (winter)
Commander Islands

Basilan Masbate

Cagayancillo Mindoro

Calayan Negros

Guimaras Palawan

Leyte Romblon

Lubang Sibuyan

Luzon Sulu

Maestro de Tawi Tawi

Campo

\section{India}

Moluccas

Ceylon (winter)

Southern China (winter)

Burmese countries (winter)

British Islands (occasional)

Western and southern Europe (winter)

Central and eastern Asia (summer)

Balabac 
Family ALAUDIDAE.

ALAUDA Linn.

(Sharpe, Cat. Bds., XIII, p. 566, 1890.)

wattersi Swinh. (p. 575, pt.)

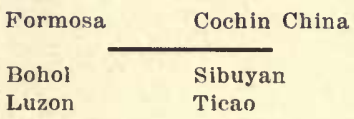

MIRAFRA Horsf.

(Sharpe, Cat. Bds., XIII, p. 593, 1890.)

philippinensis Ramsay. (p. 605.)

Luzon Mindoro
Mindanao

Family FRINGILLIDA.

LOXIA Linn.

(Sharpe, Cat. Bds., XII, p. 435, 1888.)

Iuzoniensis Grant, Bull. B. O. C., llI, p. li Iuzon (1894).

PYRRHULA Briss.

(Sharpe, Cat. Bds., XII, p. 445, 1888.)

leucogenys Grant, Bull. B. O. C., 1V, p. xli Luzon (1895).

FRINGILLA Linn.

(Sharpe, Cat. Bids., XII, p. 1\%0, 1888.)

montifringilla $\operatorname{Linn.}{ }^{\circ}$ (p. 178.)

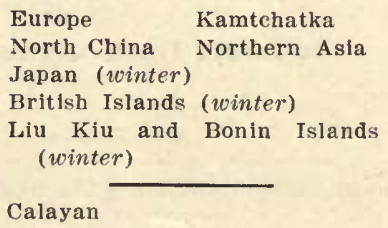


PASSER Briss.

(Sharpe, Cat. Bds., XII, p. 298, 1888.)

montanus (Linn.). (p. 30l.)

\begin{tabular}{lc} 
Malay Peninsula \\
Siam & Java \\
China & Japan \\
Europe & Persia \\
Burmah & Siberia \\
North and central Asia \\
Afghanistan & Himalayas \\
North Africa & Cochin China \\
\cline { 2 - 2 } Cebu & Luzon
\end{tabular}

SPINUS $K$ och.

(Chrysomitris Boie; Sharpe, Cat.

Bds., p. 192, 1888.)

spinus (Linn.). (p. 212.)

\author{
Northern Asla \\ British Islands \\ Japan (winter) \\ South China (winter) \\ Siberia Europe \\ Liu Kiu Islands (winter) \\ Calayan
}

EMBERIZA Briss.

(Sharpe, Cat. Bds., XII, p. 4\%6, 1888.)

spodocephala Pall. (p. 522.)

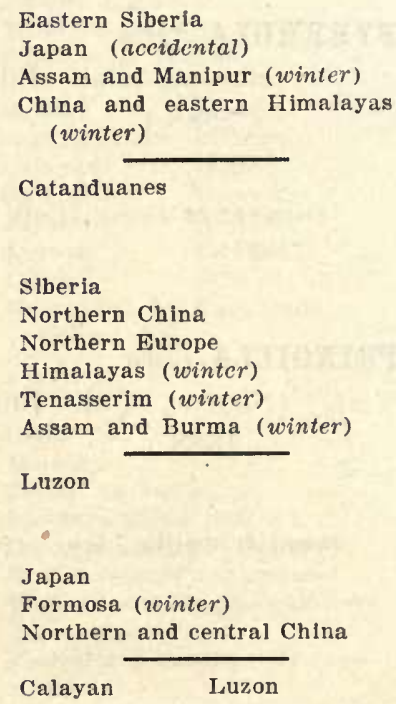

pusilla Pall. (p. 487.)

sulfurata Temm. and Schl. (p. 519.) 
CHLORURA Reichenb.

(Sharpe, Cat. Bds., XIII, p. 38s, 1890.)

brunneiventris Grant, Bull. B. O. C., III, Luzon Mindoro p. 1 (1894).

\section{Family PLOCEIDAE.}

MUNIA Hodgs.

(Sharpe, Cat. Bds., XIII, p. 326 , 1890.)

oryzivora (Linn.). (p. 328.)

\begin{tabular}{ll}
$\begin{array}{l}\text { Sumatra } \\
\text { Gava }\end{array}$ & Malacca \\
\cline { 2 - 2 } $\begin{array}{l}\text { Luzon } \\
\text { Mindanio }\end{array}$ & $\begin{array}{l}\text { Palawan } \\
\text { Panay } \\
\text { Samar }\end{array}$ \\
Balabac & Mindanao \\
Basilan & Mindoro \\
Bohol & Negros \\
Bongao & Palawan \\
Cagayan Sulu & Panay \\
Calamianes & Romblon \\
Calayan & Samar \\
Catanduanes & Semirara \\
Cebu & Sibay \\
Fuga & Sibuyan \\
Guimaras & Siquijor \\
Leyte & Sulu \\
Lubang & Tablas \\
Luzon & Tawi Tawi \\
Masbate & Ticao
\end{tabular}

formosana Swinh. (p. 338.)

Luzon

cabanisi Sharpe. (p. 353.)

Luzon

Panay

ÚROLONCHA $C a b$.

(Sharpe, Cat. Bds., XIII, p. 355, 1890.)

everetti (Tweedd.). (p. 363.)

\begin{tabular}{|c|c|}
\hline Balabac & Mindanao \\
\hline Basilan & Negros \\
\hline Calamianes & Palawan \\
\hline Calayan & Panay \\
\hline Catanduanes & Romblou \\
\hline Cebu & Samar \\
\hline Guimaras & Slbuyan \\
\hline Leyte & Tablas \\
\hline Luzon & Tawi Tawi \\
\hline Borneo & \\
\hline
\end{tabular}

${ }^{2}$ The Philippine records of Munia atricapilla were in all probability based upon specimens of $M$. jagori.-MCGREGor. 
ORIOLUS Linn.

(Sharpe, Cat. Bds., III, p. 181, 18\%\%)

chinensis $\operatorname{Limm}$. (p. 203.)

isabellæ Grant, Bull. B. O. C., IV, No. XX,

Baiabac

Basilan

Bohol

Bongao

Cagayancillo

Calamianes

Calayan

Catanduanes

Cebu

Cuyo

DInagat

Fuga

Guimaras

Lapac

Leyte

Lubang

Luzon

$M$ a estro

Campo

Marinduque

Luzon

p. ii (1894).

albiloris Grant, Bull. B. O. C., III, p. xlix (1894).

samarensis stecre, List. Bds. and Mams. Steere Exp., p. 17 (1890).

steerii ${ }^{1}$ Sharpe. (p. 213 pt.)

[basilanicus Grant, Hbis, 1896, p. 532.]

cinereogenys Bourns and Woreester, Occ. Papers Minnesota Acad., I, No. 1, p. 16 (1894).

Luzon

Leyte

Basilan

Bongao
Masbate

Mindanao

Mindoro

Negros

Palawan

Panaon

Panay

Romblon

Samar

Semirara

Sibutu

Sibuyan

Siquijor

Sulu

Tabias

Tawi Tawi

Ticao

de Verde

Sibay

Samar

Mindanao

Tawi Tawi

${ }^{1}$ Grant makes the extraordinary statement that a glance at the originąl description and figure of $O$. steerii is sufficient to show that the Negros bird is the one deseribed (Ibis, Oct., 1896, p. 532) and quotes Cat. Bds. III, p. 213, pl. $x$ (1887), in support of his contention that Bourns and 1 were mistaken in supposing that the type of $O$. steerii comes from Basilan. He therefore rejects our name of 0 . nigrostriatus for the Negros bird on the ground that it is a synonym for $O$. steerii and assigns to the Basilan bird, which he agrees with us is quite distinct from the Negros bird, the name O. basilanieus.

Had Grant followed his own suggestion and glanced at the original description of 0 . stcerii which occurs in Trans. Linn. Soc., Ser. II, Vol. I, p. 329, he would have discovered that two specimens only were collected by Steere; that one speeimen (a) was an adult male from Isabela de Basilan, while the other ( $b$ ) of which the sex was not determined, came from Negros. Also that the type described was an adult male. Furthermore, he seems to forget that the type of this species, which, by the way, still bears a label in Dr. Sharpe's well-known land, is at present in the bird eollection of the University of Michigan. It was before Dr. Bourns and myself when our description of $Q$. nigrostriatus was written. This type is an adult male from Basilan. Grant's $O$. basilunicus therefore becomes a synonym of $O$. steerii.-WoRCESTER. 
nigrostriatus Bourns and Worcester, Occ.

Masbate

Negros

Papers Minnesota Acad., I, No. 1, p. 16

(1894).

assimilis Treed., P. Z. S., 1877, p. 760.

Cebu

xanthonotus Horsf. (p. 213.)

\begin{tabular}{ll}
$\begin{array}{l}\text { Malacea } \\
\text { Java }\end{array}$ & $\begin{array}{l}\text { Sumatra } \\
\text { Borneo }\end{array}$ \\
\cline { 2 - 2 } Calamianes ?' & Palawan
\end{tabular}

Family DICRURIDA.

\section{DICRURUS Tipill.}

(Sharpe, Cat. Bis., III, p. 229, 18\%.)

balicassius (Limm.). (p. 230.)

$\begin{array}{ll}\text { Lubang } & \text { Mindanas } \\ \text { Luzon } & \text { Verde } \\ \text { Marinduque } & \end{array}$

striatus Theed. P. Z. S., 1877, p. 545.

$\begin{array}{ll}\text { Mindanae } & \text { Nipa } \\ \text { Basilan } & \text { Panaon } \\ \text { Leyte } & \text { Samar }\end{array}$

suluensis Hartert, Nor. Zool., IX, p. 441, ex Sulu

Dubois, Syu. Av., p. 1105.

mirabilis Wald. and Layard. (p. 231.)

$\begin{array}{ll}\text { Cebu } & \text { Negros } \\ \text { Gulmaras } & \text { Panay } \\ \text { Masbate } & \text { Tlcao }\end{array}$

CHIBIA Hodgs.

(Sharpe, Cat. Bds., III, p. $234,18 \%$ )
palawanensis (Tweedd.), P. Z. S., 1878, p. 614 .
Balabac
Palawan
Calamianes
cuyensis MeGregor, Bull. Philippine Mus., Cuyo No. 1, p. 5 (1903).
worcesteri MLGregor, Govt. Lab. Publ. No. Semirara 34 , p. $26(1905)$.

borneensis sharpe, I'. Z. S., 1877, p. 246.

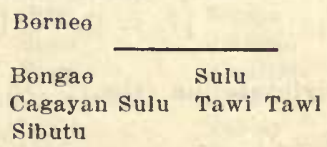

menagei Bourns and Worcester, Occ. Papers

Tablas Minnesota Acad., I, No. 1, p. 15 (1894).

${ }^{1}$ An Oriolus, in all probability (O) xanthonotus, was seen by me in the Calamianes Islands in January, 1903, but no specimens were collected.-Worcester. 
BUCHANGA Hodgs.

(Sharpe, Cat. Bds., III, p. 245, 187\%.)

palawanensis Whitehead, Ibis, 1893, p. 47. Palawan

Family STURNIDFE.

SPODIOPSAR Sharpe.

(Sharpe, Cat. Bds., XIII, p. 40, 1890.)

[Poliopsar Sharpe nec Cassin; Spodiopsar Sharpe, Ibis, 1889, p. 580. Cf. Cat. Bds., XIII, p. 665.]

sericeus $(G m$.$) . (p. 44.)$

Calayan

STURNIA Less.

(Sharpe, Cat. Bds., XIII, p. 68, 1890.)

philippensis (Forster). (p. 70.) [violacea

(Bodd.) ; cf. McGregor, Govt. Lab. Publ. No. 25, p. $30(1905)$.

Japan

Borneo (winter)

Celebes (winter)

Moluccas (vinter)

Calayan Negros

Luzon Palawan

Mindanao Tawl Tawi

Mindoro

sinensis (Gm.). (p.68.)

Formosa

Malacca (winter)

Slam

Pegu (winter)

South China (winter)

Cochin China (winter)

China

Hainan

Calayan Luzon

FTHIOPSAR Sharpe.

(Acridotheres Vieill. pt. ; Sharpe, Cat.

Bds., XIII, p. 79 pt., 1890.)

[t. c. p. 23 (footnote).]

cristatellus (Gm.). (p. 92.)

Central and southern China

Luzon (introduced)

G00DFELLOWIA Hartert.

(Hartert, Bull. B. O. C., XIV, No. C, p. 11, 1903.)

miranda Hartert. (1. c.)

Mindanao 
SARCOPS Wulden.

(Sharpe, Cat. Bds., XIII, p. 96, 1890.)

calvus (Linn.). (p. 97.)

$\begin{array}{ll}\text { Basiian } & \text { Negros } \\ \text { Bongao } & \text { Panay } \\ \text { Catanduanes } & \text { Rombion } \\ \text { Cebu } & \text { Samar } \\ \text { Dinagat } & \text { Semirara } \\ \text { Guimaras } & \text { Sibay } \\ \text { Leyte } & \text { Sibutu } \\ \text { Lubang } & \text { Sibuyan } \\ \text { Luzon } & \text { Siquijor } \\ \text { Maestro de } & \text { Sulu } \\ \text { Campo } & \text { Tablas } \\ \text { Marinduque } & \text { Tawi Tawi } \\ \text { Masbate } & \text { Ticao } \\ \text { Mindanao } & \text { Verde } \\ \text { Mindoro } & \end{array}$

EULABES $C u v$.

(Mainatus Less; Sharpe, Cat. Bds., XIII, p. 98, 1890.)

palawanensis (Sharpe). (p. 104.)

Palawan

\section{LAMPROCOCORAX $B p$.}

(Sharpe, Cat. Bds., XIII, p. 13\%, 1890.)

[Calornis Gray nec Billberg; Cf. Oberholser, Proc. Philad. Acad. Sci., 1899, p. 215.]

panayensis (Scop.). (p. 147.)

$\begin{array}{ll}\text { Agutaya } & \text { Mindanao } \\ \text { Balabac } & \text { Mindoro } \\ \text { Basilan } & \text { Negros } \\ \text { Bobol } & \text { Nipa } \\ \text { Bongao } & \text { Palawan } \\ \text { Cagayanciilo } & \text { Panay } \\ \text { Cagayan Sulu } & \text { Rombion } \\ \text { Calamianes } & \text { Samar } \\ \text { Camiguin } & \text { Semirara } \\ \text { Catanduanes } & \text { Siasi } \\ \text { Cebu } & \text { Sibutu } \\ \text { Cuyo } & \text { Sibuyan } \\ \text { Fuga } & \text { Siquijor } \\ \text { Guimaras } & \text { Suiu } \\ \text { Leyte } & \text { Tablas } \\ \text { Luzon } & \text { Tawi Tawi } \\ \text { Marinduque } & \text { Ticao } \\ \text { Masbate } & \end{array}$

todayensis Mearns, Proc. Biol. Soc. Wash., Mindanao XVIII, p. 88 (1905). 
Family CORVIDAE.

CORONE KaUp.

(Sharpe, Cat. Bds., III, p. 30, 18\%\%)

philippina (Bonap.). (p. 42.)

\begin{tabular}{|c|c|}
\hline Basilan & Mindoro \\
\hline Bohol & Negros \\
\hline Cagayancillo & Palawan \\
\hline Calayan & Panaon \\
\hline Camiguin & Panay \\
\hline Catanduanes & Romblon \\
\hline Cebu & Samar \\
\hline Cuyo & Semirara \\
\hline Dinagat & Sib \\
\hline Fuga & Sibutu \\
\hline Guimaras & Sibuyan \\
\hline Lubang & Siquijor \\
\hline Luzon & Sulu \\
\hline $\begin{array}{c}\text { Maestro de } \\
\text { Campo }\end{array}$ & $\begin{array}{l}\text { Tablas } \\
\text { Tawi Taw }\end{array}$ \\
\hline Marinduque & Ticao \\
\hline Masbate & Verde \\
\hline
\end{tabular}

CORVUS Linn.

(Sharpe, Cat. Bds., III, p. 13, 18\%\%.)

pusillus Tweedd., P. Z. S., 1878, p. 622.

Balabac Mindoro

Calamianes Palawan

samarensis Steere, List. Bds. and Mams. Samar Steere Exp., p. 23 (1890).

\section{ADDENDA.}

Add on page 101:

flava Linn. (p. 516.)

\begin{tabular}{ll} 
Africa \\
Siberia to China \\
Molueca Islands \\
Alaska & \multicolumn{1}{l}{ Europe } \\
\cline { 2 - 2 } Balabac & Masbate \\
Bohol & Mindoro \\
Calayan & Negros \\
Catanduanes & Palawan \\
Lubang & Sulu \\
Luzon & Ticao
\end{tabular}

Note.-The addition of Motacilla flava, inadvertently omitted from its proper place on page 101, raises the total number of species recorded to 692 . 


\section{INDEX TO GENERA AND SPECIES.}

abba (Calidris), 27.

Abrornis, 76 .

Acanthopneuste, 90 .

Accipiter, 41.

Acridotheres, 108.

Acrocephalus, 87 .

Actitis, 26.

acuminata (Heteropygia), 28.

acuta (Dafila), 38.

Egialitis, 23.

Egithina, 78.

renea (Muscadivora), 11.

xruginosum (Piprisoma), 98.

ieruginosus (Circus), 41.

Ethiopsar, 108.

Athopyga, 98.

affinis (Batraclostomus), 51.

affinis (Penelopides), 56.

affinis (Terpsiplıone), 75 .

Alauda, 103.

albigularis (Rhinonyias), 7.5 .

albiloris (Oriolus), 106.

albiventris (Hyloterpe), 93.

albiventris (Rlipidura), 74 .

Alcedo, 52.

Alcyone, 52.

alexandrina (Fgialitis), 24.

Alophonerpes, 66 .

Alseonax, 71.

alterum (Eloliisoma), 77 .

anabilis (Pardaliparus), 93.

Amaurornis, 17.

americanus (Merops), 56.

amethystina (Phapitreron), 10.

amurensis (Butorides), 34 .

anæstheta (Sterna), 20.

Anas, 37.

Ancylochilus, 28.

Anlinga, 39.

Anous, 21.

Anthracoceros, 55.

Anthreptes, 100.

Anthus, 101.

Antigone, 31.
Anuropsis, 81.

apicalis (Loriculus), 50 ).

apo (Dicæum), 96.

apoensis (Hyloterpe), 93.

aquila (Fregata), 40.

Arachnothera, 100.

arcuata (Dendrocygna), 37.

Ardea, 32.

ardens (Pyrotrogon), 60.

Ardetta, 35.

Arenaria, 22.

argentata (Alcyone), 52.

ariel (Fregata), 40.

arolasi (Athopyga), 98.

arquata (Numenius).

Artamides, 76.

Artamus, 91.

assimilis (Dicaeum), 96 .

assimilis (Oriolıs), 107.

Astur, 41.

atra (Fulica), 19.

atricapilla (Munia), 105.

atricapilla (Pitta), 68.

aureiloris (Zosterops), 95.

aurora (Cinnyris), 99.

avensis (Geociclıla), 84.

axillaris (Osmotreron), 9.

baclia (Spilornis), 42.

ballicassius (Dicrurus), 107

bangueyensis (Spilotreron), 11.

banyumas (Cyornis), 71 .

basilanica (Ceyx), 53.

basilanica (Muscicapula), 72 .

basilanica (Penelopides), $\mathbf{5 6}$.

basilanica (Ptilocichla), 81 .

basilanica (Zosterops), 95.

basilanicus (Oriolus), 106.

basilanicus (Polioloplıus), 79.

basilanicus (Yungipicus), 65 .

Batrachostomus, 50.

Baza, 44.

bella (Asthopyga), 98.

bella (Cyanoptila), 73 .

bengalensis (Alcedo), 52. 
besti (Dicxum), 97.

bicolor (Chimarrhornis), 85 .

bicolor (Merops), 56.

bicolor (Myristicivora), 12.

bicolor (Prionochilus), 97.

Bolbopsittacus, 49.

boltoni (Ethopyga), 98.

Bonapartea, 80.

bonapertei (Loriculus), 50.

bonga (Dicxum), 96.

bonita (Nthopyga), 98.

borcalis (Acanthopneuste), 90 .

boreotis (Sterna), 20.

borneensis (Chibia), 107 .

Botaurus, 36 .

bourusi (Ceyx), 63.

bournsi (Loriculus), 50.

Brachypteryx, 83.

brevipes (Heteractitis), 26.

brevirostris (Plapitreron), 10.

brunneiceps (Braclyypteryx), 83.

brunneiceps (Munia), 105.

brunneiceps (Phapitreron), 10.

brunneiventris (Chlorura), 10.5.

Bubo, 46.

Bubulcus, 35 .

Buchanga, 108.

Budytes, 101.

burbidgei ('Tanygnathus), 49.

Butastur, 43.

Butorides, 34 .

cabanisi (Munia), 105.

Cacatua, 48.

Cacomantis, 62.

cærulescens (Edoliisoma), 77 .

cagayanensis (Anthreptes), 100.

cagayanensis (Mixornis), 82.

calayensis (Otus), 46.

Calidris, 27.

Callaeops, 74 .

Callene, 83.

Calliope, 85.

calliope (Calliope), 85.

Callisitta, 94.

Calnenas, 15.

Calornis, 109.

calvus (Sarcops), 109.

candida (Strix), 48.

canorus (Cuculus), 61 .

canturiens (Horornis), 90.

capensis (Rostratula), 29.

capitalis (Zosterornis). 82.

caprata (Pratincola), 86.

Caprimulgus, 57. carbo (Phalacrocoprax), 39.

carola (Ptilocolpa), 12.

('arpophaga, 11.

castaneiceps (Orthotomus), 88.

caudatus (Pseudotharrhaleus), 80.

cebuensis (Artamides), 77.

cebuensis (Cittocincla), 86.

cebuensis (Collocalia), 59.

cebuensis (Cryptoloplia), 75 .

celebensis (Chatura), 59.

Centropus, 63.

cephalomelas (Cephalophoneus), 92.

Cephalophoneus, 92.

Cerchneis, 45.

cervinus (Antlıus), 102.

Cettia, 90.

ecylonensis (Culicicapa), 75.

Ceyx, 53.

Chrtura, 59.

('hacococeyx, 62.

Chalcophaps, 14.

Chalcostetha, 98.

Charadrius, 22.

Chelidon, 69.

Chelidonaria, 69.

Chibia, 107.

chinensis (Oriolus), 106.

Chimarrhornis, 85.

chirurgus (Hydrophasis), 30.

chlorigaster (Anthreptes), 100.

chloris (Halcyon), 55.

chloronotus (Orthotomus), 88.

Chloropsis, 78.

chloropus (Gallinula), 18.

Chlorura, 105.

Chrysocolaptes, 65.

chrysolaus. (Turdus), 84.

Chrysomitris), 104.

chrysonotus (Loriculus), 50.

cineraceus (Orthotomus), 88.

cinerea (Ardea), 32.

cinerea (Gallicrex), 18.

cinerea (Geocichla), 84.

cinerea ('Terekia), 26.

cinereiceps (Anuropsis), 81.

cinereiceps (Hemichelidon), 71.

cinereiceps (Iole), 79.

cinereiceps (Orthotomus), 88 .

einereiceps (Plupitreron), 10.

cinereifrons (Pycnonotus), 80.

cinereigulare (Dicæum), 97.

cinereogenys (Oriolus), 106.

cinereus (Pericrocotus), 77.

cincreus (Poliolimnas), 17. 
cinnamomea (Ardetta), 35 .

ciunamomeus (Hypocryptadius), 96.

cinnamomeus (Zeocephus), 84.

Cinnyris, 99.

circia (Querquedula), 38.

Circus, 40.

Cisticola, 89.

cisticola (Cisticola), 89.

Cittocincla, 86.

Climacteris, 95.

Clivicola, 69.

clypeata (Spatula), 38 .

Coccystes, 60 .

colestis (Cyanomyias), 73 .

Collocalia, 58.

Columba, 13.

comata (Macropteryx), 58.

communis (Falco), 44.

Copsychus, 86.

coromandelianus (Nettopus), 36.

coromandus (Bubuleus), 35.

coromandus (Coccystes), 60 .

coromandus (Halcyon), 54.

Corone, 110.

Corvus, 110.

Cotile, 69.

Cranorrhinus, 56.

crassirostris (Tringa), 28.

crecea (Nettion), 38.

Criniger, 80.

crinigera (Phlogœnas), 15.

cristata (Otomela), 92.

cristatellus (Athiopsar), 108.

Cryptolopha, 75, 76 .

cuculoides (Astur), 41.

Cuculus, 61 .

Culicicapa, 75.

cumingi (Lepidogrammus), 64 .

cumingi (Megapodius), 7.

cuyensis (Chibia), 107.

euyensis (Otus), 46.

cyanciceps (Prioniturus), 49.

cyanescens (Zeoceplus), 74.

cyaniceps (Rhipidura), 74 .

cyanogaster (Irena), 79.

cyanomelæna (Muscicapa), 73.

cyanomelas (Muscicapa), 73.

Cyanomyias, 73.

cyanopectus (Alcyone), 52.

Cyanoptila, 73.

cyanopus (Numenius), 24.

Cyornis, 71 .

Dafila, 38.

damacensis (Limonites), 28 .
Dasycrotapha, 81 .

Dasylophus, 64 .

dasypus (Chelidonaria), 69.

davao (Dicæum), 96.

Demiegretta, 33.

Dendrobiastes, 72 .

Dendrocygna, 36 .

Dendrophila, 94.

dennistouni (Zosterornis), 82 .

derbianus (Orthotomus), 88 .

Dicæum, 96.

Dicrurus, 107.

dificilis (Artamides), 76 .

dilutior (Arachnothera), 100.

dinagatensis (Cinnyris), 99.

discurus (Prioniturus), 49.

Dissöura, 31.

dominicus (Charadrius), 22.

dorsale (Dicæum), 96.

Dryococcyx, 64.

dubia (\#gialitis), 23.

dubia (Chætura), 59.

Dupetor, 36 .

dussumieri (Streptopelia), 13.

dybowskii (Locustella), 87.

Edoliisoma, 77.

Egretta, 33.

ellæ (Irena), 79 .

Elanus, 44.

elegans (Pardaliparus), 94.

elusum (Edoliisoma), 77.

Emberiza, 104.

enganensis (Cyornis), 71 .

Enneoctonus, 91.

episcopus (Dissöura), 31 .

Erithacus, 85.

ernesti (Faleo), 44.

erythaca (Cyornis), 72.

erytlirocephalus (Chrysocolaptes), 65.

erythrogaster (Pitta), 68.

erytlirogenys (Microlierax), 44.

Eudrepanis, 99.

Eudynamis, 62.

euerythra (Ceyx), 53.

Eulabes, 109.

Eumyias, 76.

eurhinus (Totanus), 25.

Eurystomus, 51.

eurythmus (Nannocnus), 35.

euryzonoides (Rallina), 16.

everetti (Dicrum), 97.

everetti (Edoliisoma), 77.

everetti (Iole), 79 .

everetti (Ninox), 47. 
everetti (Osmotreron), !).

everetti (Otus), 46.

everetti ('Tanygnathus), 49.

everetti ('Tiga), 65.

everetti (Urolonclia), 105.

everetti (Zosterops), 95.

Excalfactoria, 7 .

excellens (Cinnyris), 99.

exilis (Cisticola), 89.

falcata (Ptilocichla), 81.

falcinellus (Plegadis), 31.

Faleo, 44.

fallax (Hyloterpe), 93.

fasciata (Rallina), 16.

fasciata (Turnix), 8.

fasciolata (Locustella), 97.

ferruginea (Hemichelidon), 71 .

flagrans (Cinnyris), 99.

flammifera (Arachnothera), 100.

flava (Motacilla), 101, 110.

flaveola (Gerygone), 72.

flavicollis (Dupetor), 36.

flavigularis (Cryptolopha), 75 .

flavipectus (Athopyga), 98.

flavipennis (Chloropsis), 78 .

flavissiuna (Zosterops), 95.

flaviventer (Dicæum), 96.

florensis (Ninox), 47.

flumenicola (Alcyone), 53.

fluviatilis (Sterna), 20.

fluviventer (Dicæum), 96.

formosæ (Sphenocercus), 9.

formosana (Munia), 105.

francica (Salangana), 58.

frater (Criniger), 80.

frater (Eudynamis), 63.

Fregata, 40.

Firingilla, 103.

frontalis (Callisitta), 94 .

frontalis (Orthotomus), 88.

frontalis (Phapitreron), 10.

fuciphaga (Salangana), 58.

fugax (Hierococcyx), 61.

fugensis (Hypsipetes), 79 .

Fulica, 19.

fuliginosa (Sterna), 21.

fuliginosus (Microstictus), 66 .

fuliginosus (Otus), 46.

Fuligula, 39 .

fuligula (Fuligula), 39.

fulvifasciatus (Yungipieus), 65.

fulvus (Charadrius), 22.

funebris (Microstictus), 66 .

fuscans (Uroloncha), 105. fuscus (Limnobenus), 17.

galgulus (Loriculus), 50.

Gallicrex, 18.

gallinaceus (Hydralector), 30 .

Gallinago, 29.

gallinago (Gallinago), 29

Gallinula, 18.

Gallus, 8.

gallus (Gallus), 8.

Garzetta, 33.

garzetta (Egretta), 33.

Geociclıla, 84.

geoffroyi (Ochthodromus), 23.

Geopelia, 14.

Gerygone, 72.

gigantea (Clixtura), 59 .

gigantea (Pelargopsis), 52 .

Glareola, 30.

glareola (Rlyyacoplilus), 27.

Glottis, 26.

goiavier (Pycnollotus), 80.

goisagi (Gorsachius), 34.

Goodfellowia, 108.

goodfellowi (Zosterops), $\mathbf{9 6}$.

Gorsachius, 34.

gouldi (Pelargopsis), 52.

griseatus (Caprimulgus), 57.

griseigularis (Anthreptes), 100.

griseigularis (Columba), 13.

griseipectus (Pseudotharrlaleus), 81.

griseipectus (Ptilocolpa), 12.

griseisticta (Heniclıelidon), 71 .

guillemardi (Artamides), 76.

guimarasensis (Cinnyris), 99.

guimarasensis (Iole), 79.

gularis (Aecipiter), 41.

gularis (IIalcyon), 54.

gurneyi (Pseudoptynx), 46.

gustavi (Anthus), 102.

guttata (Dendrocygna), 37.

guttulata (Dendrocygna), 37 .

gutturalis (Hirnndo), 70 .

Gymnoerotaphus, 80.

Gymnolxmus, 56.

hæmatocephalum (Xäntlolæun), 64.

hæmatostictım (Dicæum), 96.

hæmatribon (Chrysocolaptes), 65.

hrematuropygia (Cacatua), 48.

Haleyon, 54.

Haliaëtıs, 43.

laliaëtus (Pandion), 45.

Haliastur, 43.

hancei (Oreocichla), 84.

hargitti ('Tluriponax), 66, 67. 
Harpactes, 60.

laaringtoni (Dryococeyx), (j4.

haynaldi (Iole), 79.

helen:e (Cyanomyias), 73.

helianthea (Culicicapa), 75.

Helodromas, 25.

helvetica (S'puatarola), 22.

Hemichelidon, 70 .

Hemilophus, (66.

herioti (Cyornis), 71 .

Herodias, 33.

Heteractitis, 26.

heterolamus (Plıyllergates), 91.

Heteropygia, 28.

Hierococcyx, 61.

Hinantopus, 24.

Hirundo, 69.

lolospilus (Spilornis), 42.

hombroni (Halcyon), 55.

homeyeri (Hyloterpe), 93.

honorata (Eudynamis), 62.

Horornis, 90.

humilis (Onopopelia), 14.

hybrida (Hydrochelidon), 20.

Hyclralector, 30 .

Hydrochelidon, 20.

Hydrocorax, 55.

hydrocorax (Hydrocorax), 55.

Hydrophasis, 30 .

Hyloterpe, 93.

Hypocryptadius, 96.

hypoleucum (Dicæum), 97 .

lypoleucus (Actitis), 26.

hypoleucus (Elanus), 44.

Hypotanidia, 15 .

Hypothymis, 73.

Hypsipetes, 79.

ichthyaëtus (Polioxtus), 45.

igneus (Pericrocotus), 77.

lladopsis, 81.

indica (Chalcophaps), 14.

indicus (Butastur), 43.

indicus (Limonidromus), 101.

inexpectata (Salangana), 59.

inexpectatus (Prionochilus), 97.

inornata (Rhabdornis), 95.

insignis (Chalcostetha), 98 .

insignis (Rhinomyias), 75 .

intermedia (Mesophoyx), 32.

intermedia (P’orzana), 15.

intermedium (1)icamm), 96.

internedius (Bolbopsittacus), 49.

intermedius (Cuculus), 61.

intermedius (Haliastur), 43. interpres (Arenaria), 2:.

interpres (Geocichla), 84.

Iole, 79.

Irena, 79 .

isabellæ (Oriolus), 106.

jagori (Munia), 105.

japonicar (Ninox), 47.

javanica (Butorides), 34.

javanica (Hirundo), 70.

javanicus (Centropus), 6:3.

javensis (Batrachostomus), 51.

javensis (Thriponax), $66,67$.

jefleryi (Eudrepanis), 99.

jelleryi (Pithecophaga), 42.

johanna (Prionochilus), 97.

johnstonix (Trichoglossus), 48.

jotaka (Caprimulgus), 57.

jugularis (Cinnyris), 99.

juliæe (Cinnyris), 99.

keayi (Plilogonas), 15.

kelleri (Merula), 83.

kettlewelli (Macronus), $\mathbf{8 2}$.

kieneri (Lophotriorchis), 42.

kochi (Artamides), 76.

kochi (Pitta), 68.

Lalage, 78.

Lamprococorax, 109.

Lamprotreron, 11.

lanceolata (Locustella), 87.

langhornei (Muscadivora), 11.

Lanius, 91, 92.

Larus, 21.

latirostris (Aleseonax), 71.

leclancheri (Leucotreron), 10.

lemprieri (Cyornis), 72.

lempricri (Gymnolæmus), 56.

Leonardia, 83.

Leonardina, 83.

Lepidogrammus, 64 .

leucocephala (Pelargopsis), 5l.

lcucocephalus (Cranorrhinus), 56.

leucocephalus (Himantopus), 24.

leucocephalus (Pandion), 45.

lencogaster (Artamus), 91.

leueogaster (Haliaëtus), 43.

lencogenys (Pyrrhula), 103.

lencomelas (1'uftinus), 19.

lencopais (Baza), 44.

leucoptera (Hyclrocheliclon), 20.

lencorhynchus (Artamus), 91.

leucostriatus (Budytes), 101.

leucotis (l'hapitreron), 10.

Lencotreron, 10.

leytensis (Pericrocotus), 77. 
leytensis (Yungipicus), $6 \mathbf{5}$.

lilacea (Callisitta), 94.

Limicola, 29.

limnætus (Spizëtus), 42.

Limnobxnus, 17.

Limonidromus, 101.

Limonites, 27.

Limosa, 25.

limosa (Limosa), 25.

linchi (Salangana), 59.

lindsayi (Halcyon), 55.

lineata (Excalfactoria), 7 .

Locustella, 87 .

longicornis (Otus), 46.

Lophotriorchis, 42.

Joriculus, 50.

lowi (Salangana), 58.

I.oxia, 103.

lucidus (Chrysocolaptes), 65.

lucionensis (Otomela), 92.

lucionensis ('Tanygnathus), 49.

luconensis (Prioniturus), 49.

lugubris (Acanthopneuste), 90 .

lugubris (Ninox), 47 .

Jugubris (Surniculus), 60.

lunulatus (Bolbopsittacus), 49.

Lusciniola, 88.

luzonica (Anas), 37.

luzonica (Phlogœenas), 15.

luzonica (Zosterops), 95.

luzoniense (Dicæum), 96.

luzoniensis (Cittocinela), 86.

luzoniensis (Loxia), 103.

luzoniensis (Muscicapula), 72.

luzoniensis (Penthornis), 94.

Iyncornis, 57.

Macronus, 82 .

macroptera (Ninox), 47.

Macropteryx, 58 .

Macropygia, 13.

macrotis (Lyncornis), 57.

macrurus (Caprimulgus), 57.

macrurus (Megalurus), 89.

maculatus (Antlius), 101.

maculatus (Yungipicus), 65.

maculipectus (Phapitreron), 10.

magnifica ( Athopyga), 98.

magnirostris (Baza), 44.

magnirostris (Orthorhamphus), 30 .

Mainatus, 109.

major (Hyloterpe), 93.

major (Macropterix), 58.

malaccensis (Anthreptes), 100.

Malacocincla, 81 .
Malacopterum, 81.

malamaui (Ceyx), 53.

malayanus (Chalcococcyx), 62.

malayensis (Anthus), 102.

manilla (Petrophila), 85.

manillæ (Penelopides), 56 .

manillensis (Accipiter), 41.

manillensis (Caprimulgus), 57.

manillensis (Nycticorax), 34.

manillensis (Pyrrherodias), 32.

marchesa (Pericrocotus), 77 .

marchei (Gymnolæmus), 56.

marchei (Leucotreron), 10.

Mareca, 37.

marginata (Salangana), 59.

meyeni (Zosterops), 95.

megala (Gallinago), 29.

megalotis (Otus), 46.

Megalurus, 89.

Megapodius, 7 .

melanauchen (Sterna), 21.

melanoceplalus (Microtarsus), 80 .

melanochlamys (Irena), 79.

melanogaster (Anhinga), 39.

melamogynys (Falco), 45.

melamoleuca (Lalage), 78 .

melauoleucus (Cireus), 41.

melanolophus (Gorsachius), 34.

melanope (Motacilla), 101.

melanops (Centropus), 64.

melanura (Ceyx), 53.

menagei (Batrachostomus), 5 ].

menagei (Chibia), 107.

menagei (Phlogœenas), 15.

nienagei (Yungipicus), 65.

meninting (Alcedo), 52.

meridionalis (Microhierax), 44.

Merops, 56.

Merula, 83.

merulinus (Cacomantis), 62 .

mesoleuca (Callisitta), 94.

Mesophoyx, 32.

Mesoscolopax, 25.

Microhierax, 44.

micropterus (Cuculus), 61.

Micropus, 80.

microrhynchus (Batrachostomus), 50.

Microtarsus, 80.

Microstictus, 66 .

mindanense (Dicæum), 97.

mindanense (Fdoliisoma), 77 .

mindanensis (Artamides), 76 .

mindanensis (Bolbopsittacus), 49.

mindanensis (Brachypteryx), 83. 
mindanensis (Caprimulgus), $\tilde{\mathrm{z}}$.

mindanensis (Ceyx), 53.

mindanensis (Copsychus), 86 .

mindanensis (Cryptolopha), 76 .

mindanensis (Eudynammis), 63.

mindanensis (Hydrocorax), 55.

mindanensis (Lyncornis), 57.

mindanensis (Maeronus), 82.

mindanensis (Muscicapula), 72 .

mindanensis (Pardaliparus), 94.

mindanensis (Ptilocichla), 81.

nindorensis (Artamides), 77.

mindorensis (Centropus), 63.

mindorensis (Hyloterpe), 93.

mindorensis (Iole), 79 .

mindorensis (Loriculus), 50.

mindorensis (Merula), 83.

mindorensis (Ninox), 47.

mindorensis (Otus), 46.

mindorensis (Penelopides), 56.

mindorensis (Prioniturus), 49.

mindorensis (Thriponax), 66, 67 .

mindorensis (Zonophaps), 12.

minor (Lalage), 78.

minor (Rhabdornis), 95.

minuta (AEthopyga), 98.

minuta (Horornis), 9 l.

minuta (Limonites), 27.

minutus (Mesoscolopax), 25.

minuta (Ptilocichla), 81 .

mirabilis (Dicrurus), 107.

Mirafra, 103.

miranda (Goodfellowia), 108.

Mixornis, 82.

modesta (Dafila), 38.

mongolus (Ochtlodromus), 23.

montani (Anthracoceros), 55.

montanus (Macronus), 82.

montanus (Passer), 104.

montanus (Prioniturus), 48.

monticola, 85 .

monticola (Iole), 79 .

montif ringilla (Fringilla), 103.

montigena (Muscicapula), 72.

montis (Cryptolopha), 76.

moseleyi (Halcyon), 55.

Motacilla, 100.

mülleri (Pitta), 68.

Mulleripicus, 66.

Munia, 105.

Muscadivora, 11.

Muscicapa, 73 .

Muscicapula, 72 .

Myristicivora, 12. mystacalis (Rhabdornis), 95.

napoleonis (Polyplectron), 8.

narcissina (Xanthopygia), 73.

Nannocnus, 35.

nasutus (Cephalophoneus), 92 .

nebularius (Glottis), 26.

nehrkornæ (Polyplectron), 8 .

nehrkorni (Micropus), 94.

Nettion, 38.

Nettopus, 36.

nicobarica (Calœnas), 15.

niger (Lalage), 78.

nigra (Cittocincla), 86 .

nigriceps (Orthotomus), 88.

nigrilore (Dicæum), 97.

nigriloris (Eumyias), 76 .

nigrimentalis (Eumyias), 76.

nigrirostris (Alcyone), 53.

nigritorquis (Rlipidura), 74 .

nigrocapitata (Zosterornis), 82.

nigrocinnamomea (Rhipidura), 74 .

nigrorum (Abrornis), 76.

nigrorum (Cittocincla), 86.

nigrorum (Merula), 83.

nigrorum (Muscicapula), 72 .

nigrorum (Phapitreron), 10.

nigrorum (Ptilocolpa), 12.

nigrorum (Zosterops), 95.

nigrostriatus (Oriolus), 106, 107.

Ninox, 47.

nipalensis (Treron), 9.

novæ-zealandiæ (Limosa), 25.

novus (Pericrocotus), 77.

nuchalis (Muscadivora), 11.

Numenius, 24.

Nycticorax, 33.

nyeticorax (Nycticorax), 33.

obscurior (Cinnyris), 99.

obscurum (Dicæum), 97.

obscurus (Turdus), 84.

occipitalis (Hypothymis), 73 .

occipitalis (Leucotreron), 10 .

occipitalis (Plıapitreron), 10.

Oceanodroma, 19.

ocellata (Turnix), 8.

ochotensis (Locustella), 87.

ochropus (Helodromas), 25.

Octhodromus, 23.

ocularis (Motacilla), 100.

ocularis (Rhinomyias), 75 .

cnanthe (Saxicola), 86.

cenanthoides (Saxicola), 86.

cenochlamys (Callisitta), 94.

olivacea (Abrornis), 76. 
olivacea (Amaurornis), 17. olivaceus (Prionochilus), 97. Onopopelia, 14.

Oreociclila, 84 .

orientalis (Acrocephalus), 87.

orientalis (Eurystomus), 51 .

orientalis (Glareola), 30.

Oriolus, 106.

Orthorhamphus, 30 .

Orthotomus, 88.

oryziola (Cisticola), 89.

oryzivora (Munia), 105.

Osmotreron, 9.

Otomela, 92 .

Otus, 46.

oustaleti (Strix), 48.

Pacliycephala, 93.

Pachycephalixus, 80 .

palawanense (Malacopterum), 81.

palawanensis (Buchanga), 108.

palawanensis (Chibia), 107 .

palawanensis (Chloropsis), 78 .

palawanensis (Criniger), 80 .

palawanensis (Eulabes), 109.

pallidior (Dicæum), 96.

pallidior (Tachornis), 59.

pallidus ('Turdus), 84.

palustris (Megalurus), 89 .

panayense (Edoliisoma), 77.

panayensis (Artamides), $7 \pi$.

panayensis (Culicicapa), 75 .

panayensis (Eumyias), 76 .

panayensis (Lamprococorax), 109.

panayensis (Orthotomus), 88 .

panayensis (Spilornis), 42.

Pandion, 45.

panini (Penelopides), 56.

papuense (1)icxum), 96.

papuensis (Pipra), 96.

Pardaliparus, 93.

Parus, 93, 94.

Passer, 104.

paykulli (Limmobenus), 17 .

pectoralis (Thriponax), $66,67$.

pelargopsis, 51.

Pelecanus, 40.

penelope (Mareea), 37.

Penelopides, 56 .

Penthoceryx, 6l.

Penthornis, 94.

peregrims (Falco), 44.

Pericrocotus, 77 .

periophthalmica (Callaeops), 74 .

Perinis. 44. peroni (Aegialitis), 23.

Petrophila, 85.

phe (Macropygia), 13.

lhalacrocorax, 39 .

Phapitreron, 10.

philippensis (Iole), 70 .

philippensis (Loriculus), 50.

philippensis (Ninox), 47 .

philippensis (Pelecanus), 40.

philippensis (Sturnia), 108.

philippensis (Thriponax), 66,67 .

philippina (Corone), 110.

philippinensis (Arachnother:a), 100.

philippinensis (Cyornis), 72.

philippinensis (Hyloterpe), 93 .

philippinensis (Hypotmidia), 16.

philippinensis (Mirafra), 103.

philippinensis (Jodicipes), 19 .

philippinensis (Pseudoptynx), 46.

philippinensis (Spizaëtıs), 42 .

philippinus (Merops), 57.

philippinus (Phyllergates), 91 .

Plilogøenas, 15.

phonicura (Amaurornis), 18.

Phoyx, 32.

phragmitoides (Cisticola), 89.

l'hyllergates, 91.

Phyllobates, 91.

Phylloscopus, 90.

picina (Chætura), 59.

pickeringi (Muscadivora), 11.

pileatus (Halcyon), 54.

Pipra, 96.

Piprisoma, 98.

piscator (Sula), 39 .

Pithecophaga, 42.

Pitta, 68.

Plotus, 39.

platenæ (Cyomis), 72.

platenæ (Y'hlogonas), lŏ.

plateni (Hyloterpe), 93.

plateni (Ninox), 47.

plateni (Zosterornis), 82.

platyrhyncha (Limicola), 29.

Plegadis, 31 .

plumbea (Porzana), 16.

Podicipes, 19.

Polioaëtus, 45.

poliocephala (Zonophaps), 12.

poliogyna (Brachypteryx), 8:3.

Poliolimnas, 17.

Poliolophus, 79.

Polionetta, 37.

Poliopsar, 108. 
pollens (Artamicles). 76 .

Polyplectron, 8.

Porphyrio, 18.

Porzana, 16.

Pratincola, 86.

Prioniturus, 48.

Prionochilus; 97.

propinqua (Pitta), 68.

Pseudoptynx, 46.

Pseudotharrhaleus, 80.

Ptilocichla, 81.

Ptiloeolpa, 12.

ptilonorhynehus (Pernis), 44.

Puffinus, 19.

pulcherrima (Eudrepanis), 99.

pulverulentus (Mulleripicus), 66 .

pulverulentus (Porphyrio), 18.

punetatus (Megalurus), 89.

pusilla (Emberiza), 104.

pusillai (Porzana), 16.

pusillus (Corvus), 110.

Pycnonotus, 80.

pygmxa (Zosterornis), 82.

pygmæum (Dicæum), 97.

Pyrotrogon, 60.

Pyrrherodias, 32.

Pyrihula, 103.

quadricolor (Prionochilus), 97.

Querquedula, 38 .

querquedula (Querquedula), 38.

Rallina, 16.

ramsayi (Cyornis), 72.

ramsayi (Yungipícus), 65.

regulus (Loriculus), 50.

retrocinctum (Diceum), $\mathbf{9 6 .}$

reyi (Ninox), 47 .

Rlabdornis, 95 .

Rhinomyias, 75 .

Rhipidura, 74.

rhizophorge (Gerygone), 72.

rhodolæma (Anthreptes), 100.

Rhyacophilus, 27.

richardi (Anthus), 102.

richmondi (Zosterops), 95.

ridibundus (Larus), 21.

riparia (Clivicola), 69.

romblonis (Otus), 46.

roseum (Xantholrma), 64.

Rostratula, 29.

rubrinota (Athopyga), 98.

rubriventer (Dicrum), 96.

refuscens (Otus), 46.

ruficauda (Rhinomyias), 75.

ruficeps (Megalurus), 89. luficeps (Orthotomus), 88.

ruficollis (Limonites), 27.

rufifrons (Turdinus), 81.

rufigularis (Iole), 79 .

rufopunctatus (Chrysocolaptes), 65.

rufulus (Anthus), 102.

rufus (Zeocephus), 74 .

rustica (Hirundo), 69.

sacra (Demiegretta), 33.

Salangana, 58 .

samarensis (Ceyx), 53.

samarensis (Corvus), 110.

samarensis (Hypothymis), 73 .

samarcnsis (Muscicapula), 72 .

samarensis (Oriolus), 106.

samarensis (Orthotomus), 88 .

samarensis (Penelopides), 56.

samarensis (Rhinomyias), 75 .

samarensis (Sareoplanops), 6 is.

Sarcophanops, 68.

Sarcops, 109.

saturatus (Cuculus), 61.

sauli (Rhipidura), 74.

Saxicola, 86.

schalowi (Cephalophoneus), 92.

schimackeri (lole), 79.

seutulata (Ninox), 47.

seebohmi (Horornis), 90.

seebohmi (Tribura), 88.

semigaleatus (Hydrocorax), 55.

semilarvatus (Penthornis), 94.

septimus (Batrachostomus), 50.

sericeus (Spodiopsar), 108.

severus (Falco), 45.

sliarpei (Antigone), 31.

shelleyi (Athopyga), 98.

sibirica (Hemichelidon), 70 .

sibutuense (Dicæum), 97 .

sibutuensis (Otus), 46.

sibuyanicum (Dicæum), 96 .

simplex (Cyornis), 72.

simplex (Gerygone), 72.

sinensis (Ardetta), 35.

sinensis (Centropus), 63.

sinensis (Clivicola), 69.

sinensis (Sterma), 21.

sinensis (Stumia), 108.

Siphia, 71.

siquijorensis (Iole), 79.

siquijorensis (Ioriculus), 50.

siquijorensis (Zosterops), 95.

Sitta, 94.

solitaria (Petrophila), 85.

soloensis (Astmr), 41. 
sonnerati (Penthoceryx), 61. sorgophilus (Acrocephalus), 87. sparveroides (Hierococcyx), 61. Spatula, 38.

speciosa (Dasycrotapha), 81. sperata (Cinnyris), 99.

Sphenocercus, 9.

Spinus, 104.

spinus (Spinus), 104.

spilocephala (Ninox), 47.

spilonota (Ninox), 47.

spilonotus (Circus), 40.

Spilopelia, 14.

Spilornis, 42.

Spilotreron, 11.

Spizaëtus, 42.

Spodiopsar, 108.

spodocephala (Emberiza), 104.

Squntarola, 22.

steerii (Alcyone), 52.

steerii (Centropus), 63.

steerii (Oriolus), 106.

steeri (Pitta), 68.

steerei (Sarcophanops), 68.

stellaris (Botaurus), 36.

stenura (Gallinago), 29.

Sterna, 20.

Stictognathus, 80.

stolidus (Anous), 21.

Stoparola, 76 .

Streptopelia, 13.

striata (Geopelia), 14.

striata (Hypotænidia), 15.

striata (Zosterornis), 82.

striaticeps (Iole), 79 .

striaticeps (Macronus), 82.

striatus (Artamides), 76.

striatus (Dicrurus), 107.

striolata (Hirundo), 70.

Strix, 48.

Sturnia, 108.

subarquatus (Ancylochilus), 28. sumatrana (Ardea), 32.

Sula, 39.

sula (Sula), 40.

sulfurata (Emberiza), 104.

suluensis (Cephalophoneus), 92 .

suluensis (Dicrurus), 107.

suluensis (Prioniturus), 49.

suluensis (Thriponax), 66, 67 .

suluensis (Turnix), 8.

superciliaris (Cittocincla), 86.

superciliaris (Hypothymis), 73.

superciliosa (Otomela), 92, 93. superciliosus (Dasylophus), 64 .

Surniculus, 60.

Syrnium, 47.

tabuensis (Porzana), 16.

Tachornis, 59.

talisi (Penelopides), 56.

'anygnathus, 49.

Tatare, 87.

temmincki (Lamprotreron), 11.

temmincki (Limonites), 28.

tenuirostris (Macropygia), 13.

terat (Lalage), 78.

'Terekia, 26.

lerpsiphone, 75.

thomassoni (Merula), 83.

'Ihriponax, 66.

'liga, 65.

tigrina (Spilopelia), 14.

tigrinus (Enneoctonus), 91.

timoriensis (Herodias), 33.

tinnunculus (Cerchneis), 45.

todayensis (Lamprococorax), 109.

torquata (Hypotænidia), 16.

Totanus, 25.

'I'reron, 9.

'Tribura, 88.

'Trichoglossus, 48.

Tringa, 28.

Tringoides, 26.

trivirgatus (Astur), 41.

troglodytes (Salangana), 59.

Turdinus, 81.

Turdus, 84.

Turnix, 8.

tweeddalii (Irena), 79.

unicolor (Pseudotharrhaleus), 80.

unirufus (Centropus), 64.

Uroloncha, 105.

urostictus (Poliolophus), 79.

validirostris (Cephalophoneus), 92.

validirostris (Yungipicus), 65.

varia (Oreocichla), 84.

variegatus (Numenius), 24.

vegæ (Larus), 21.

velutinus (Surniculus), 60.

veredus (Ochthodromus), 23.

vernans (Osmotreron), 9.

verticalis (Prioniturus), 48.

viduata (Dendrocygna), 36 .

violacea (Sturnia), 108.

viridis (Agithina), 78.

viridis (Centropus), 63.

vulcani (Zosterops), 96 .

waldeni (Cranorrhinus), 56. 
waterstradti (Prioniturus), 49. wattersi (Alauda), 103.

whiteheadi (Cinnyris), 99.

whiteheadi (Salangana), 58.

whiteheadi (Hyloterpe), 93.

whiteheadi (Otus), 46.

whiteheadi (Syrnium), 47.

whiteheadi (Turnix), 8.

whiteheadi (Zosterops), 95.

whiteheadi (Zosterornis), 82.

wiepkeni (Syrnium), 47.

wiglesworthi (Anthreptes), 100.

winchelli (Halcyon), 54.

winchelli (Hyloterpe), 93.

woodi (Leonordina), 83.

woodi (Mixornis), 82.

worcesteri (Chibia), 107. worcesteri (Loriculus), 50.

worcesteri (Turnix), 8.

westermanni (Muscicapula), 72.

xanthocephalus (Chrysocolaptes), 65.

xanthodryas (Acanthopneuste), 90.

Xantholæma, 64.

xanthonotus (Oriolus), 107.

Xanthopygia, 73.

xanthopygia (Cryptolopha), 67 .

xanthopygium (Dicæum), 97.

xanthorhynchus (Chalcococeyx), 62.

Yungipicus, 65.

Zeocephus, 74.

Zonophaps, 12.

zonorhyncha (Polionetta), 37.

Zosterops, 95.

Zosterornis, 82. 


\section{INDEX TO ORDERS, SUBORDERS, FAMILIES, AND SUBFAMILIES.}

Accipitres, 40.

Accipitriformes, 40.

Accipitrinæ, 40.

Acromyodi, 69.

Alaudidx, 103.

Alcedinidæ, 51.

Alcedininæ, 51.

Anatida, 36.

Anatinre, 36.

Anseriformes, 36 .

Aquilinæ, 42.

Ardeæ, 32.

Ardeidar, 32.

Ardeiformes, 31 .

Arenariinæ, 22.

Artamidse, 91.

Brachypteryginæ, 83.

Bubonidæ, 46.

Buboninæ, 46.

13ucerotes, 55.

Bucerotidx, 55.

Cacatuidæe, 48.

Calœenadinæ, 15.

Campophagidæ, 76 .

Capitonida, 64.

Capitones, 64.

Caprimulgi, 57.

Caprimulgidx, 57.

Caprimulginæ, 57.

Carinatæ, 7.

Carpophaginæ, 11.

Centropodinæ, 63.

Certhiidæ, 95.

Chaeturinæ, 58.

Charadrii, 22.

Charadriidx, 22.

Charadriiformes, 22.

Charadriinse, 22.

Ciconix, 31.

Ciconiidæ, 31.

Ciconiinæ, 31 .

Columber. 9.
Columbidse, 13.

Columbiformes, 9.

Columbinæ, 13.

Coracix, 51.

Coraciidx, 51.

Coraciiformes, 50.

Coraciinse, 51.

Corvidæ, 110.

Cuculi, 60.

Cuculidæ, 60.

Cuculinæ, 60.

Cursorii, 30.

Cypseli, 58.

Cypselidx, 58.

Cypselinx, 59.

Daceloninæ, 53.

Dicaeidx, 96 .

Dicruridse, 107.

Eurylaemidæ, 68.

Eurylaemiformes, 68.

Eurylaeminæ, 68.

Falconidx, 40.

Fringillidre, 103.

Fregatidx, 40.

Fulicinæ, 19.

Fuligulinæ, 39.

Galliformes, 7 .

Geopeliinæ, 14.

Geotrygonina, 15.

Glareolidæ, 30.

Grues, 31.

Gruidæ, 31.

Gruiformes, 31.

Halcyones, 51.

Hemipodii, 8 .

Himantopodinæ, 24.

Hirundinidx, 69.

Hirundinina, 69.

Ibididx, 31.

Laniidre, 91 .

Laniina, 91 .

Laricle, 20. 
Lariformes, 20.

Jarinx, 21.

loriidx, 48.

Macropterygidx, 58 .

Macropygiinæ, 13.

Megapodii, 7.

Megapodiidx, 7 .

Meropes, 50.

Meropidæ, 56 .

Mesomyodi, 68 .

Motacillida, 100.

Muscicapidæ, 70.

Nectarini idæ, 98.

Oedicnemi, 30.

Oedienenid $x$, 30 .

Pacliycephalinxe, 93.

P'alaeolnithinæ, 48.

Pandiones, 45.

Pandionidæ, 45.

Paridæ; 93.

Parinæ, 93.

Parræ, 30.

Parriclæ, 30.

Passeriformes, 68.

Pelecanidx, 40.

l'elecaniformes, 39 .

Peristeridæ, 13.

Phabinæ, 14.

Phalacrocoracicla, 39.

Pliasiani, 7.

Plasianidre, 7.

Phoenicophainæe, (j4.

Pici, 65.

Picidæ, 65.

Piciformes, 65.

Picinæ, 65.

Pittidx, 68 .

Platalex, 31 .

Plectropterina, $\mathbf{3 6 .}$

Ploceidx, 105.
Plotidx, 39.

Podargi, 50.

Podargidæ, 50.

Podicipedidæ, 19.

Podicipedidiformes, 19.

Procellariidæ, 19.

Procellariiformes, 19.

P'rocellariinæ, 19.

Psittacidx, 48.

Psittaciformes, 48.

Ptilopodinæ, 10.

Puffinidæ, 19.

l'uffininx, 19.

Pycnonotidx, 78.

Rallidæ, 15.

Ralliformes, 15.

Rallinæ, 15.

Ruticillinæ, 85.

Scansores, 64.

Scolopacinæ, 27.

Sittidx, 94 .

Sterninæ, 20.

Sturnida, 108.

Strigidæ, 48.

Strigiformes, 46 .

Sulidæ, 39.

Sylviidæ, 87 .

Syrniinæ, 47 .

Timeliidx, 80.

Timeliinæ, 80.

Totaninæ, 24.

Treronidæ, 9.

Treroninæ, 9.

Trogones, 60.

Trogonidæ, 60 .

Turdidæ, 83.

Turdina, 83.

Turnicidre, 8.

Turturinæ, 13.

Zosteropida, 95. 






